
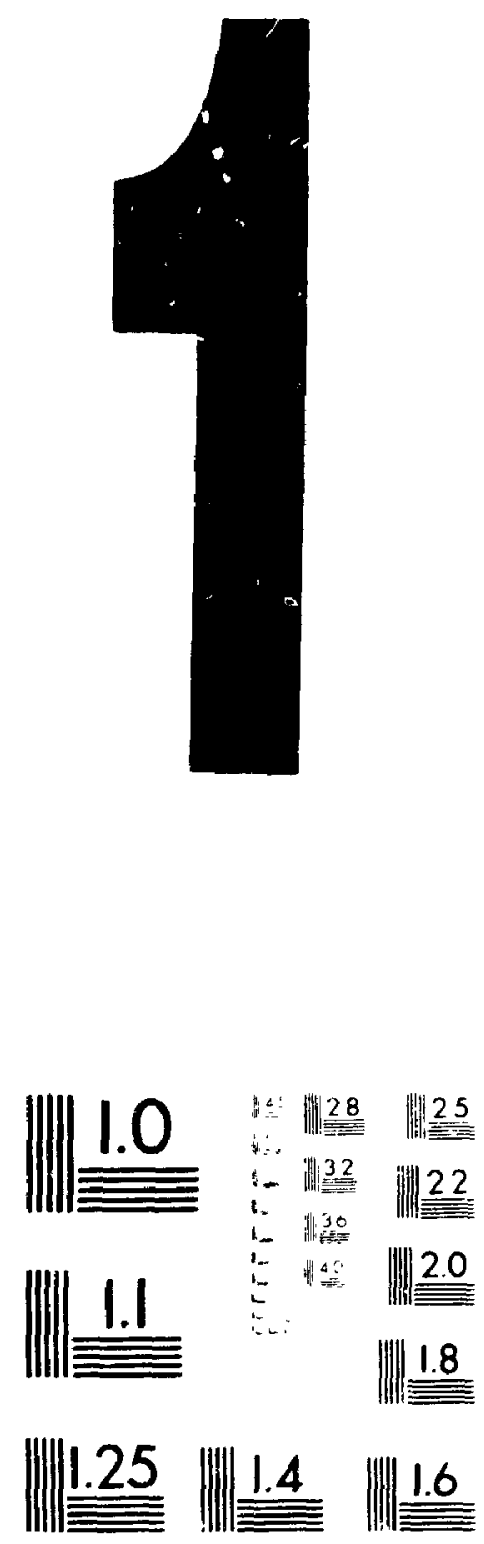

MICROCOPY RESOLUTION TEST CHART

NAT1, NA! EIIHEAU (IF ,TANDAGLS

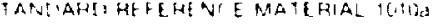

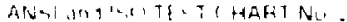


NOTICE

The quality of this microform is heavily dependent upon the quality of the original thesis submitted for microfilming. Every effort has been made to ensure the highest quality of reproduction possible.

If pages are missing, contact the university which granted the degree.

Some pages may have indistinct print especially if the original pages were typed with a poor typewritei ribbon or if the university sent us an inferior photocopy.

Reproduction in full or in part of this microform is governed by the Canadian Copyright Act, R.S.C. 1970, c. C-30, and subsequent amendments.
I.a qualité de cette microforme dégjend grandement de la qualité de la thèse soumise au microfiiimage. Nous avons tout fait pour assurer une qualité supérieure de reproduction.

S'il manque des pages, veuillez communiquer avec l'université qui a conféré le grade.

La qualité d'impression de certaines pages peut laisser à désirer, surtout si les pages originales ont áté dactylographiées à l'aide d'un ruban usé ou si l'université nous a fail parvenir une photocopie de qualité inférieure.

La reproduction, même partielle, de cette microforme est soumise à la Loi canadienne sur le droit d'auteur, SRC 1970, c. C-30, et ses amendements subséquents. 
Electrophysiological Investigation of the Dorsal Nucleus of the Lateral Lemniscus in the Auditory system of the Albiro Rat

by

Athena Despina Buckthought, B.SC., B.A.

A thesis submitted to

the Faculty of Graduate Studies and Research

in partial fulfillment of

the requirements for the degree of

Master of science

Department of Psychology

Carleton University

Ottawa, Ontario

September, 1993

C1993 Athena Despina Buckthought 
Acquisitions and

Bibliographic Services Branch

395 Wellingtun Street

Ottawa. Ontario

KIA ON4
Direction des acquisitions et

des services bibliographiques

395. rue Wellinglon

OHawa (Ontario)
The author has granted an irrevocable non-exclusive licence allowing the National Library of Canada to reproduce, loan, distribute or sell copies of his/her thesis by any meaus and in any form or format, making this thesis available to interested persons.
L'auteur a accordé une licence irrévocable et non exclusive permettant à la Bibliothèque nationale du Canada de reproduire, prêter, distribuer ou vendre des copies de sa thèse de quelque manière et sous quelque forme que ce soit pour mettre des exemplaires de cette thìse à la disposition des personnes intéressées.
The author retains ownership of the copyright in his/her thesis. Neither the thesis nor substantial extracts from it may be printed or otherwise reproduced without his/her permission.
L'auteur conserve la propriété du droit d'auteur qui protège sa thèse. Ni la thèse ni des extraits substantiels de celle-ci ne doivent être imprimés ou autrement reproduits sans son autorisation. 
Nume $: i$ :

Dissertotion Abstracts international is ar ranged by broad, general subject categories Please select the one subject which most neurly describes the content of your dissentation Enter the corresponding four-digit code in the spaces provided

\section{Subject Colegories}

\section{THE HUMANITIES AND SOCIAL SCIENCES}

\section{COMMUMICATIONS AND THE ARTS}

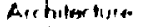

A.t Hiales

rinerre

[xume:

Firie Aarts

Jomereslism

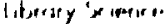

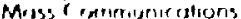

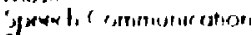

\section{coucation}

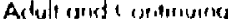

Ayrirulleural

Art

Busirienss

(ommining ( ollecy.

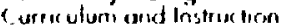

carlyc hutiotorom

Clemernetheny

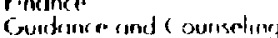

Hextiti

Higher

Home l corkennice

Inichustind

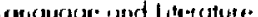

Mrithemellic:

Must

ihysical

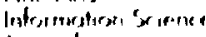

Phitosophy of

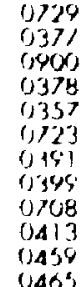

(1) 4505

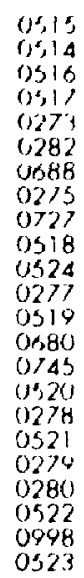

Psycholesyy

Reording

Reirgious

Scienres

Secoridary

Social Sciences

Srroculogy of

speriol

Peacher Training

Techrinloyy

Meosurements

Vorutional

MANGUAGE, LITERATURE AND

LINGUISTICS

lanquage

Generd
Arscien:

linguisfics

Modern

Liserature

Clossical

Comparative

Medievo

Modern

Alricon

Asisin

Conodian (English)

Cunadian ifrench.

English

Germonic

Lalin American

Middle Eustern

Romonse

S'avie und East Europear.

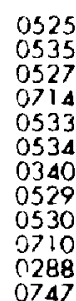

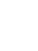

0679

0289

0290

0291

0401

0294

0295

0297
0298

0298

0591

0305

0352
0355
0593

0593

0311
0312

0312

0315
0313

0314
PHILOSOPHY, RELIGION AKD

THEOLOGY

Philosophy

Religion

Biblical Studies

Clergy

Pistory of

Theology

\section{SOCIAL SCIENCES}

American Studies

Anthropology

$$
\text { Archoeol }
$$

Phisical

Business Administration

Generd

Accountsng

Management

Markefing

Canodian Studies

Economics

Generd

Agricultural

Commerre Busiriess

Finonce

History

Theory

Folkiore

Geography

Gerontology

Genero
0422

0318

0321

0319

0322

0322
0469

0323

0324

0326

0327

0310

0272

0770

0454

c385

0501

0503

0505

0508

0509

0510

0511

0358

0351

0578

\section{THE SCIENCES AND ENGINEERING}

\section{Dological sciences}

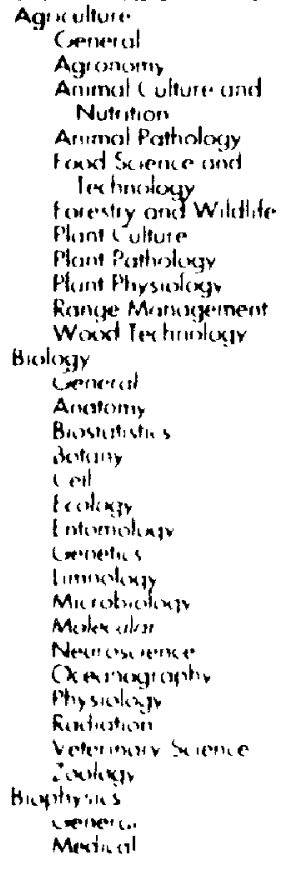

EARTH SCEENES

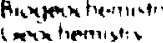

\section{Georlesy}

ceolcyy

Hydrology

Minesa! gy

Poleverotony

Poleoniology

Poleoroology

Palynology

Physis al Geography

Phvsical Ocenrography

HEALTH AND EAVIRONHENTAL

\section{SCIENCES}

Emiromentai Scrences

Hewlth Sxiences

General

Chemothersupy

Deentistry

Hosptol Atonogement

Hum in Development

Hum'sn Deve
imminology

Mental therglth

Nu' ing

Obstetrics and Gynerology

Occupotionol trealth and

Thergpy

f'attrology

Pharmacology

Phrimucy

Public Heateh

Rodiology
Rex reotion
Medicire and Surgery

0370

0372

0373

0388

0411

0426

0418

0985

0427

0368

0415

$3 \mathrm{ke}$
$\mathrm{in}$

inds
Speech Pathology
Toxicology
Home Economics

PhYsical sciences

Pure Sciences

Chemistry

General

Analyical

Brochemistry

Inorganic

Nuclear

Pharmaceutical

Physical

Polyme

Radiation

Matherna
Phirsics

General

Acoustics

Astronomy arid

Astrophysics

Airrospheric Science

Alamic

Electroniss and Electricing

Elementary Particles and

High Energy

Flued ard Plasmo

Molecular

Nurlea

Opies

Rodiation

Statistics

Applied Sciences

Applied Mechunics

Compuler Science

0460

0386

0485

0749

0486

0487

0738

0490

0491

0.994

0495

0754
0405

0405

0605
0986

0006

0508

07.88

0607

0798
0759

0609

0610

0752

0756

0011

0463 
The undersigned recommend to the Faculty of Graduate studies and Research acceptance of the thesis

Electrophysiological Investigation of

the Dorsal Nucleus of the Lateral Lemniscus

in the Auditory System of the Albino Rat

submitted by Athera Despina Buckthought, B.A., B.Sc.

in partial fulfillment of the requirements for

the degree of Master of Science

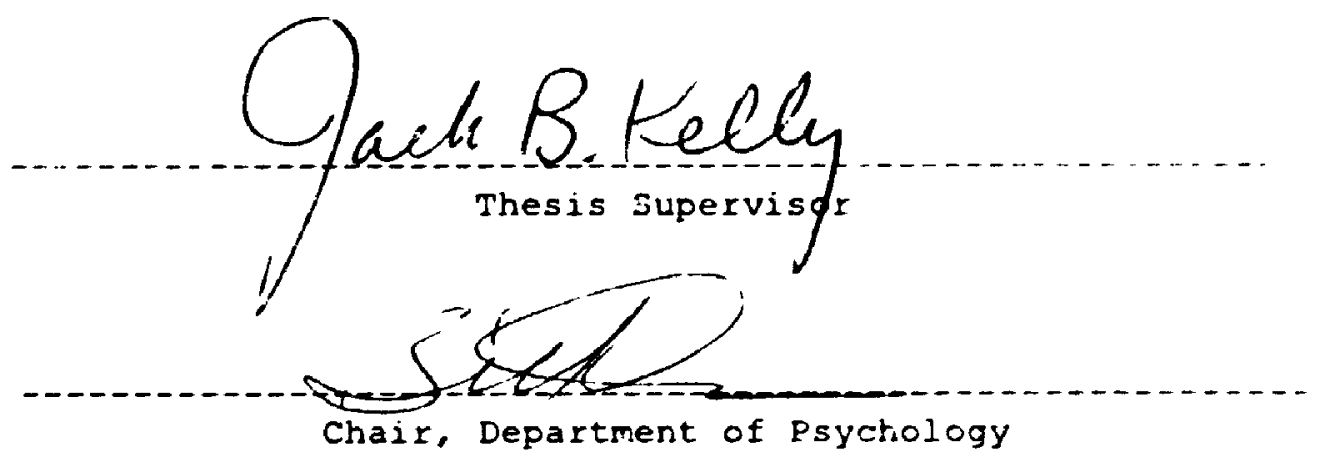

Carleton University

September, 1993 
Abstract:

Responses of single neurons to binaural tone burst stimulation were recorded with microelectrodes from the dorsal nucleus of the lateral lemniscus (DNLL) in pentobarbizal-anesthetized rats. Responses were also obtained from the intermediate and ventral nuclei of the lateral lemniscus (INLL, VNLL), sagulum (SAG), and paralemniscal zone.

Electrode positicns were verified using dye injections, or electrolytic lesions.

Post-stimulus response patterns in DNLL included onset, sustained and onset-pause-sustained responses to contralateral stimulation. Most neurons (744, $N=100$ ) were excited by contralateral stimulation and inhibited by ipsilateral stimulation (EO/I suppression responses), and were sensitive to small interaural intensity differences (IID). IID curves were similar to those for inferior colliculus neurons. Fewer neurons showed only monaural sensitivity $(17$ EO/O), or were excited by stimulation of elther ear (9.0z EE).

The eagulur: also showed binaural sensitivity !91.8* EO/I, $N=111$, but most INLL units showed only monaural sensitivity $(62.9 \div E 0 / 0, \mathrm{~N}=$ 35).

The data are consistent with the idea that DNLL pizys an important role in binaural processing witnin the auditory system. 


\section{Acknowledgements}

Foremost I thank my supervısor, Dr. Jack Kelly, for his continued encouragement and support, for his openness to different ideas, and for his expert advice and guidance in all matters of experimental research.

I thank Brian van Adel, Bryan Rooney, Liang Li, and shu Hul Wu for providing a hospitable and intellectually stimulating lab environment in which to work. I especially thank Bryan Rooney for taking the time to glve advice and help when he was so busy himself, and Briar van Adel for help that went beyonc the requirements of his job, and fo-many thought-provoking discussions.

I thank friends and family for persoral support during times i had to work lorg hours in order to complete experiments and subsequent datd analysis. In particular, I thank Nelson for helping me out in numerous ways, and I thank my parents for encouraging me in the pursuit of higter educatjor.

I gratefilly acknowledge that the stereotaxic co-ordinate system used to localize the Drll in oblique electrode tracks in thas study was developed and provided by Liang $L 1$. 


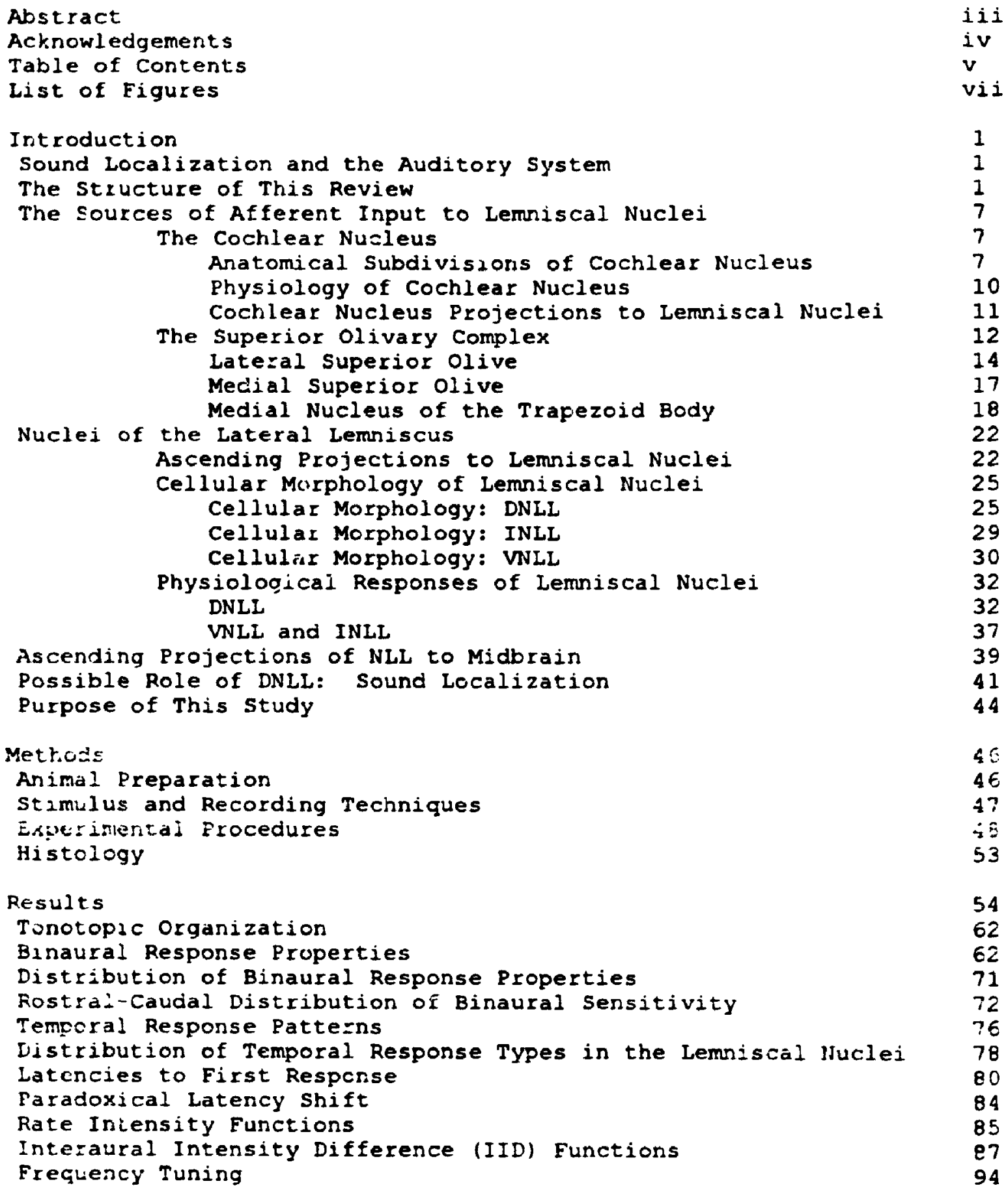


$\begin{array}{ll}\text { Discussior: } & 09 \\ \text { DNLI } & 90 \\ \text { Areas Dorsal to DNLL } & 102 \\ \text { Rostral DNLL } & 103 \\ \text { INLI } & 104 \\ \text { Paralemniscal Zone } & 104 \\ \text { VNLI } & 110 \\ \text { Sagulum } & 110 \\ \text { Summary and Implications for Future Research } & 11 . \\ \text { References } & 114 \\ & \\ \text { Appendix A: List of Abbreviations } & 128 \\ \text { Appendix B: Preparation of Equithesin } & 1311 \\ \text { Appendix C: Distribution of Characteristic Frequencies } & 131 \\ & 133 \\ \text { Appendix D: Post-Stimulus Histograms } & 1.33\end{array}$ 


\section{List of Eigures:}

Figure 1. Ascending auditory projections from the cochlear nucleus, which terminate in the superior olivary complex, the nuclei of the lateral lemnisc $s$, and the inferior colliculus.

Figure 2. Generalized schematic sumary of ascending auditory system projections, excluding minor pathways.

Figure 3. Schematic diagrams summarizing ascending projections to the nuciel of the lateral lemniscus from the cochlear nucleus and superior olivary complex.

Figure 4. Frontal sections showing reconstructed vertical and oblique electrode tracks, with characteristic frequencies and binaural iesponse type of neurons recorded along each track shown.

Figure 5. Composite reconstructions in which data from all expe:imental animals (except rostal and caudal cases) were pooled and pajected to a single representative frontal sertion, to illustrate distribution of (A) characteristic frequencies, (B) binaural response types, (C) temporal response types within the lemniscal ruclei.

Figure 6. Percentages of (A) binaural response types or (B) temporal response patterns obtained in DNLL, INLL and sagulum. 
Figure 7. Percentage of different binaural response types found along the restrocaudal axis through DNLL.

Figure 8. Post-stimulus histograms illustrating different temporal response patterns found in DNLL or INLL.

Eigure 9. Distribution of latencies in response to pure tone bursts at CE, $10 \mathrm{~dB}$ above threshold, (A) for units located in DNLL, and (B) for different binaural response types found in DNLL.

Figurc 10. A. Distribution of latencies in response to pure tone bursts at characteristic frequency (CF) plotted as a function of CF. for neurons in DNLL, INLL, sagulum, or regions medial to DNLL and INLL (paralemniscal zone). B. Medial-lateral distribution of iatencies in DNLE ard surrounding structures.

Eigure 11. Paradoxical latency shift illustrated by the resporisc : neuron BU27-2 to contralaterally presented pure tone bursts at characteristic frequency, for two different sound pressure levels.

Figure 12. Rate intensity functions for single units in DNLL (A) and dorsal to the main division of DNLL (B) show monotonic and nonmonotonic behaviour. C. Rate intensity functions measured at different frequencies of tone burst stimulation for an onset chopper (BUS6-2) in central DNLL. 
Figure 13. Interaural intensity difference (IID) curves from EO/I units with different temporal response patserns, and varying rates of spontaneous activity, in central DNLL, INLL, and in regions dorsal to the main subdivision of DNLL.

Figure 14. Representative tuning curves for single units located in central DNLL, rostral DNLL, INLI, or regions dorsal to the main subdivision of DNLI.

Figure 1 (Appendix $C$ ). Composite reconstruction to illustrate the locations of units with characteristic frequencies in the lemniscal nurlei (same reconstruction as in Figure 5, but all lemniscai nuclei have been included).

Figure 2 (Appendix D). Post-stimulus histograms for two well-isolated single units recorded from DNLL illustrates the effects of different sound pressure levels and frequencies of tone burst (binaural or monaural) stimulation.

Table 1. Numbers of single units recorded from different regions in the lemniscal pathway: (A) different binaural response types, and (B) different temporal response patterns. 


\section{Introduction:}

\section{Sound Localization and the Auditory System:}

The auditory system differs from the other major sensory systems in the complexity of its subcortical pathways and number of processing levels. One reason for the comple.ity of auditory system is that, unlike the visual system, in which a given direction in space corresponds to a point on the retina, the auditory system must compute the direction of a sound source using mechanisms of the central nervous system. As a sound source moves avay from midline in the horizontal plane (azimuth), the sound waves arrive earlier and are more intense at one ear, giving rise to interaural time differences (ITDs) and interaural intensity differences (IIDs). Both ITDs and IIDs are dependent on sound frequency. At low frequencies, interaural phase differences (IPDS) are the important binaural localization cue: at highs frequencies, IFDs no longer unambiguously code location iriformation, ans delays in the signal envelope are used as the ITI cue. IID coding besctse tho most important cue for high frequencies.

The structure of This Review

Axons of neurons from cochlear nucleus (CN) and superior olivary complex (SOC) ascend to the midbrain inferior colliculus (IC) through the lateral lemniscus (IL) on each side of the trair (Figure 1, 2 ). Embedded witrin th: fibers of $L L$ is a band of cells, exteridirig from the lateral superior olive to the IC, which includes the dorsal, intermediate and ventral nuclel of the lateral lemriscus (UHLL, INLL, VNLl. The thil is a binaurally sensitive nucleus resporsive to IIDs and ITUs, probably important in sound localizatzon, while the Illid and 
that neurons in the lateral limb of the uso distribute projections over larger areas in the contralateral rather than ipsilateral IC, while neurons in the medial limb of LSO project to larger areas in the ipsilateral IC (Schneiderman and Henkel, 1987). However, retrograde labelling studies showed projections of the LSO to the ipsilateral and contralateral IC more uniformy distribu'ed within LSO (rat: Beyerl, 1978).

The bilateral projection of ISO to inferior colliculus is paralleled by a bilateral projection of the LSO to the dorsal nucleus of the lateral lemniscus (Elverland, 1978; Glenddening et al.. 1981). Many uso neuzons are probably GABAergic and thus have inhibitory projections; glutamate decarboxylase (GAD) positive cell bodies are found throughout LSO (rat: Moore and Moore, 1987; cat: Adams and Mugnaini, 1990: gerbil: Roberts and Ribak, 1987), and GABA-positive cell bodies have been reported (guinea pig: Helfert et al., 1989; Peyret $\in t$ al.. 1987; Thompson et al., 1985; cat: Adams and Mugnaini, 1-u; Salst-Marze ec al., 1989). That some Lso neurors are giycinergic has been shown by high levels of tritiated strychnine binding to uso demenstrated autoradiographically (Giendenning and Baker, 1988; 2arbin et $31 . .1981$, intense glycine receptor immuncreactivity in LSO (Altschuier et al., 1986), and glycine-immunoreactivity of many cell bodles in LSO (Wenthold et al., 1987). High levels of choline acetyltransferase (ChAT) enzymatic activity (Wenthold and Morest, 19761, and presence of acetylcholinesterase containing cells losen et al., 2084), may give evidence for cholinergic cells in LSO. 
display either monaural or binaural responses.

The multisynaptic pathways through NLL perallel the other ascending pathways to the inferior colliculus. The DNLL receives inputs (Figure 3) from many of the same sources as the inferior colliculus, such as ventral cochlear nucleus (VCN), lateral superior olis , (LSO), and medial superior olive (MSO), and thus, has both monaural and binaural inputs. Principal excltatory inpurs include contralateral Iso, ipsilateral MSO, and contralateral $\mathrm{CN}$, while ipsilateral so and contralateral DNLI may provide inhibitory inputs to DNLL. The DNLL projects bilaterally to the inferior colliculus.

In contrast to DNLL, both VNLL and INLï are components of $a$ monaural pathway in parallel with the binaural pathways. VNLL and INLL receive input (Eigure 3) mainly from contralateral cochlear nucleus, ard INLL receives additionai monaural inputs from ipsilateral medial nucleus of the trapezoid body (MNTB; part of the superior olive) and INLI. However, the INLI also receives minor projections from MSO ipsilaterally and LSO bilaterally, as does DNLL, suggesting that INLL might be a partly binaural nucleus. The INLL and VNLL project largely to ipsilateral inferior colliculus, in contrast to the bilateral projection of DNLL.

The nuclei of the lateral lemniscus (NLL) are unusual in receiving a diverse set of inputs, which include all divisions of cochlear nucleus (CN), and the majof binaural and monaural nuclei of the superior olivary complex (SOC). Therefore it is helpful to consider the physiological response patterns and binaural sensitivity in these nucle., as well as anatomical projections to the lemniscal nuclei, to explain the response properties of the NLL. A review of some 
information on the superior olivary complex (SOC) also makes it possible to make functional comparisons between the pathways through the NLL to the inferior colliculus, and the binaural pathways through the soc to the inferior colliculus. Therefore, before discussing the anatomical and physiologicei features of the NLL, this review will sunmarize some important aspects of the large body of anatomical and physiological data on the $\mathrm{CN}$ and $\mathrm{SOC}$. Since very little information is available on the NLL, all studies done to date will be reviewed in detail. 
Figure 1. Ascending auditory projections from the cochlear nucleus, which originate in specific regions in the cochlear nucleus (1) and terminate in the superior olivary complex (2), the nuclei of the lateral lemniscus (3), and the inferior colliculus (1). Thick, medium, and thin lines show major, moderate and minor projections, respectively. 1: Glob, globular cells; Mult, multipolar cells, Sph, spherical cells (anteroventral cochlear nucleus), Oct, octopus cells (posteroventral cochlear nucleus); Fus, fusiform cells; Giant, giant cells (dorsal cochlear nucleus); VIII, cochlear nerve root. 2: DAS, dorsal acoustic stria; DLPO, dorsolateral periclivary nucleus: DMPO, dorsomedial periolivary nucleus; LSO, lateral superior olive: LNTB, lateral nucleus of the trapezold body: MNTB, medial nucleus of the trapezoid body; MSO, medial superior olive; VMPO, ventromedial

periolivary nucleus: VAS, ventral acoustic strias VNTB, ventral nucleus of the trapezoid body. 3: DNLL, dorsal nucleus of the lateral

lemniscus; INLL, intermediate nucleus of the lateral lemniscus; VNLL, ventral nucleus of the lateral lemsiscus. A: CIC, central nucleus of the inferior colliculus; DCx, dorsal cortex (Adapted from Helfert et al., 2991).

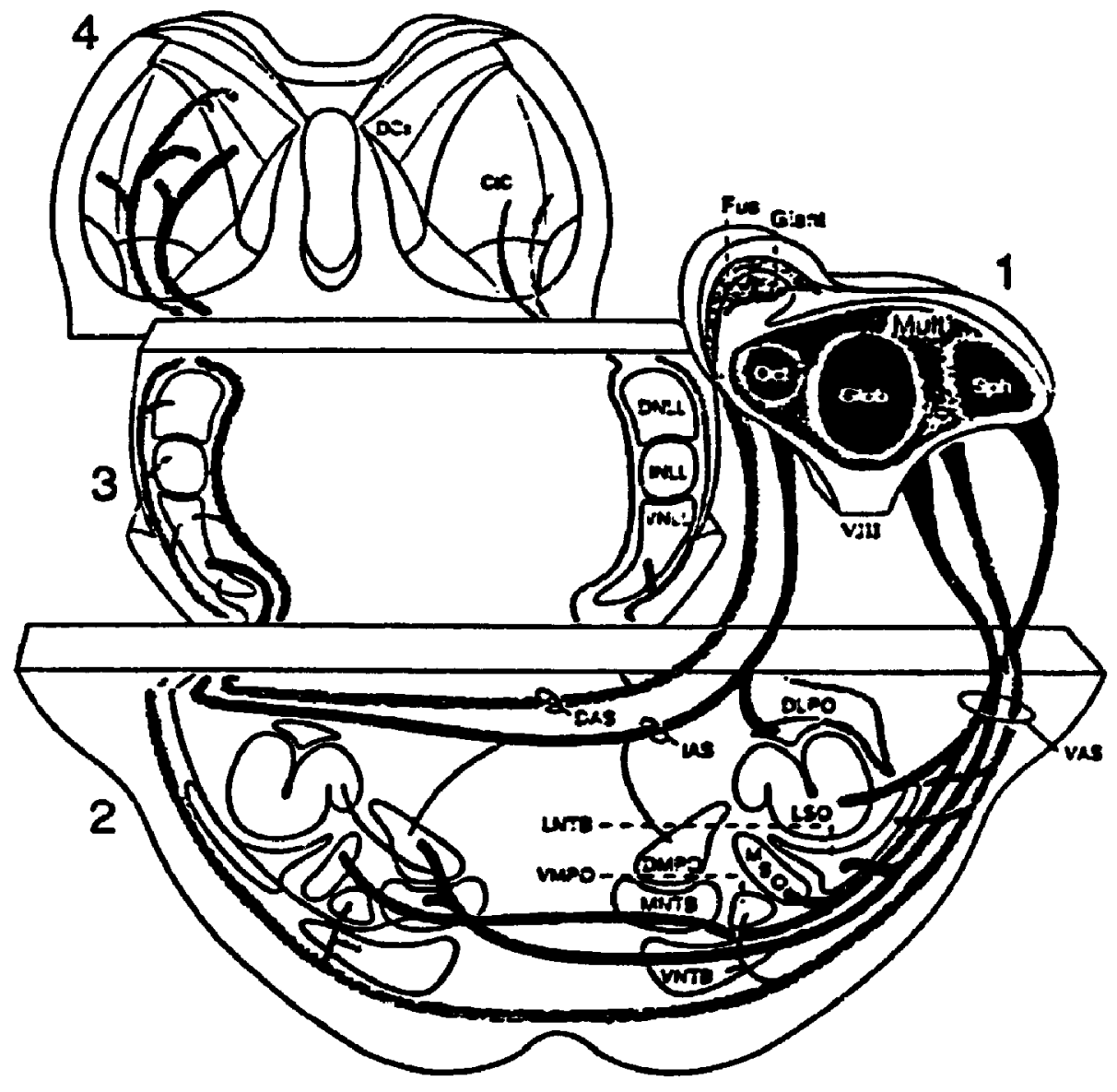


Flgure 2. Generalized schematic sumary of ascending auditory system projections, excluding minor pathways. AVCN, anteroventral cochlear nucleus; DCN, dorsal cochlear nucleus: PVCN, posteroventral cochlear nucleus; MSO, medial nucleus of the superior olive; LSO, lateral nucleus of the superior olive: MTB, medial nucleus of the trapezoid body: IC, Inferlor colliculus: MGB, medial geniculate body: NLL, nuclei of the lateral lemniscus (Adapted from Pickles, 1988).

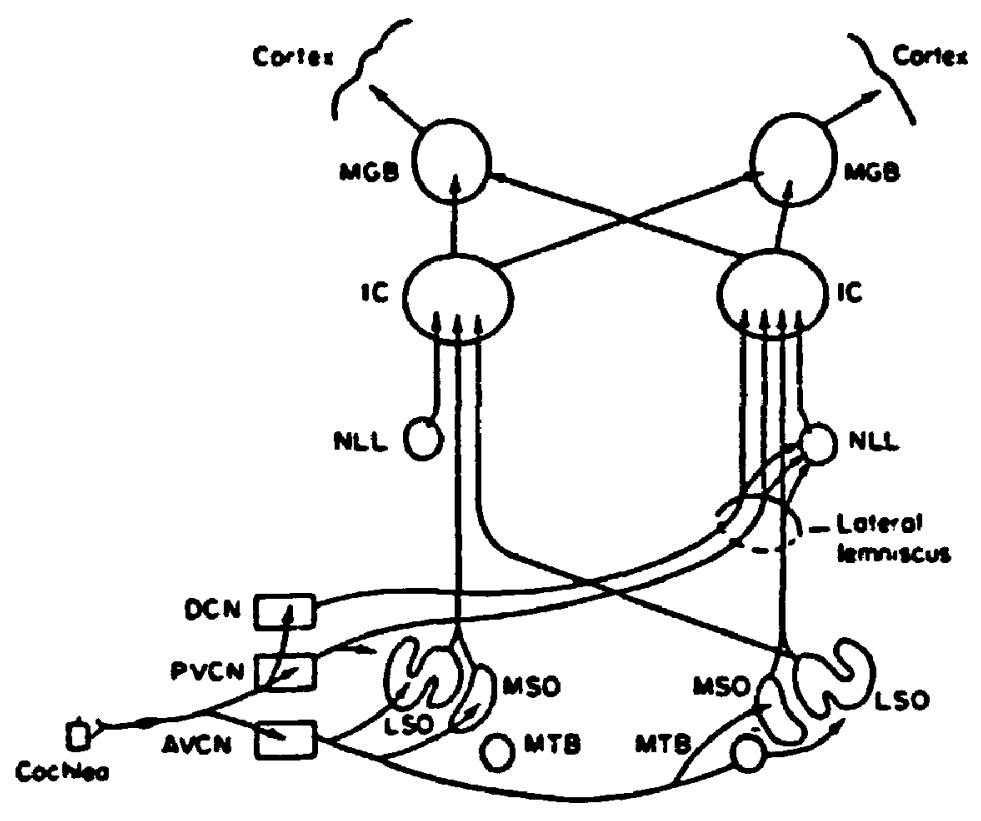


The Sources of Afierent Input to the Lemniscal Nuclei:

\section{The Cochlear Nucleus}

\section{Aratomical Subdivisions of Cochlear Nucleus (CN)}

The cochlear nucleus $(\mathrm{CN})$ has been divided into dorsal and ventral divisions (dorsal cochlear nucleus, DCN; ventral cochlear nucleus, VCN), with the ventral division further divided into anterior and posterior divisions lanteroventral cochlear nucleus, AVCN; posteroventral cochlear nucleus, PVCN). All auditory nerve fibers innervate all three of these subdivisions of the cochlear nucleus. Each auditory nerve fiber bifurcates after entering $\mathrm{CN}$, sending an ascending branch to AVCN and a descending branch which passes through PVCN (giving off collaterals) and teminates in DCN (Fekete et al.. 1984; Osen, 1970). Auditory nerve fibers send terminals to the three CN subdivisions in a strictly organized tonotopic sequence, with higher frequen:y fibers sending collaterals to the dorsocaudal regions of each subaiveicn, wille lower frequency fibers innervate more rostroveritral regioss. As a result, in either DCN, PVCN or AVCN, microelectrode peretrations from dorsocaudal to rostroventral encounter neurons with progressively higher characteristic frequency (CF) (Evans and Nelson, 1973; Goldberg and Brownell, 1973; Rose et al., 1959; Rose, 1960).

Each of these three divisions of $\mathrm{CN}$ is morphologically

heterogeneous, and receives a different pattern of innervation, and therefore can be further subdivided. Osen (1969) classified CN neurons using Nissl preparations, and proposed a division of the nucleus into nine divisions, based upon the distribution of nine major cell types. Brawer et al. (1974) proposed a different set of subdivisions of CN, 
from different neuron types identified in Golgi impregnations, but also based upon cytoarchitecture in Nissl preparations. Later studies have correlated the cell types identified in Nissl or Golgi preparations, and have made electron microscope observations on the synaptic innervation of identified neuron types. Thus, differing sets of neuron types (and names of neuronal types) in $\mathrm{CN}$ are referred to in the literature, depending on whether the characteristics used to distinguish the neurons are from Nissl preparations, Golgi preparations, or studies with the electron microscope.

Brawer et al. (1974) divided AVCN into an anterior division, which is further dirided into anterior (AA), posterior (AP), and posterodorsal (APD) parts, and a posterior division with dorsal (PD) and ventral (PV) parts. Two types of large neuron, "bushy" ana "stellate" cells are found throughout AVCN in Golgi impregnations (Brawer et al., 1974; Cant and Morest, 1979b). AA contains almost exclusivaly bushy cells, while in AP, bushy and steliate cells aze Elund in approximately equal numbers (Brawer et al.. 1074; Cant end Morest, 1979a). AA ccrresponds to Osen's (1969a, 1969t) area of lazge spherical cells, while AP corresponds to Osen's small spherical cell area. In the posterior division of AVCN, the bushy, stellate or elorgate cells (Golgi preparation) have been identified with globular and muitipolar cells (Nissl preparation), respectively (Tolbert and Mcrest, 1982a, 1982b; Tolbert et al., 1982). The posterodorsal (PD) and posteroventral (PV) parts of AVCN approximately correspond to Osen's multipolar and globular cell areas (Brawer et al., 1974!. The spherical (bushy) cells $A A$ and APD divisions of anterior AVCN probably supply the excitatory input to medial superior olivary (MSO) 
neurons that process intecaural time difference cues (EE cells) (Cant and Casseday, 1986). The spherical sushy cells of AA, APD and AP (anterior AVCN) and cells from PD (globular, multipolar or stellate) supply excitatory afferents to ipsilateral lateral superior olive (LSO) neurons which process interaural intensity differences (IE cells) (Cant and Casseday, 1986). The inhibitory input to Lso (IE) neurons, which results in these neurons being inhibited by contralateral stimulation, arises from the globular cell pathway to LNTB or MNTB from the posterior (PD or PV) division of AVCN. Likt:. se, MSO summation (EE) neurons receive an inhibitory input from the globular cell pathway through LNTB and MNTB; inhibition is necessary to the function of these neurons to produce lowered firing levels for non-optimal interaural time differences (Cant, 1991; Cant, 1984; Warr, 1982, 1972a; Tolbert et al., 1982; Harrison and Warr, 1962; Smith et al., 19871.

The posteroventral cochlear nucleus (PVCN) contains stellate cells (similar to those in AVCN) which are widely distributed within the nucieus, and octopus cells (named for their dendritic structure which gives them the appearance of an octopus), predominately found at the centre of PVCN in the octopus cell area (Brawer et al., 1974; Osen, $2969)$.

The dorsal cochlea: nucleus (DCN) has a structure which varies substantially among species, being stratified among nonprimate mamals, and unstratifled among primates (Moore, 1980; 2worykin, 1964). In the cat, there are three neural layers: an outermost layer, the "molecular layer", containing small cells and granule cells; a "fusiform cell layer" containing rows of fusiform rells, as well as small cells and granule cells; and a "polymorphic" or deep layer with giant cells, 
small cells and granule cells (Brawer et al.. 1974; Kane, 1974).

Autoradiographic (Frostrolm and Rotter, 1986; Glendenning and

Baker, 1988) and imunocytochemical studies (Juiz et al., 1989)

demonstrated that GABA is an important inhibitory neurotransmitter in

CN. Additional evidence has come from pharmacological studies

demonstrating that GABA exerts an inhibitory action on cochlear nucleus neurons (Caspary et al., 1985), and from brain slice studies (Potashner et al.. 1985; Wenthold, 1979).

studies on glycine distribution, uptake and release, and pharmacological in vivo or in vitro studies have demonstrated that CN neurons utilize the inhibitory transmitter glycine (Caspary et al.. 1985; Staatz-Benson and Potashner, 1987; 1988; Wenthold and Martin, 1984; Wu and vertel, 1986). Immunocytochemical studies localized glycine-containing cell bodies in cochlear nucleus (Peyret et al., 1987; Wenthold et al., 1987; Aoki et al., 1988) and found glyimmunoztactive cell bodies to be most abundant in DCN (Wenthold et al., :EC:i. Si: large cell types in val showed glyeine receptor immunoreactivity (Wenthold et al., 1988).

Excitatory amino acids (EEAs), including glutamate and aspartate, are probably important neurotransmitters in cochlear nucleus; EEA receptors have been localized autoradiographically in CN (Greenamyre et al.. 2984; Halpain et al., 1984; Monaghan and Cotman, 19851.

Physiology of Cochlear Nucleus (CN):

Cochlear nucleus neurons are typically monaural, responding only tn ipsilateral stimulation. Physiological response patterns of cochlear nucleus (CN) neurons, based on temporal patterns of response to short 
tone bursts (post-stimulus time histograms, PSTHs), include primarylike (PL), primarylike with notch (PIn), choppers (C), onset lockers (01), onset choppers (OC), pausers (P) and buildups (B). Primarylike units have temporal response patterns and interspike interval histograms similar to those of the (primary afferent) auditory nerve fibers. Onset units have an initial spike at stimulus onset, followed by low or null discharge rate. Choppers respond to tonal stimulation with several bursts of spikes separated by intervals that are unrelated to stimulus frequency. Onset choppers respond with two to four bursts of spikes at the onset of a tone. A pauser unit has one or two initial spikes, followed by a 5-100 msec silent period which is followed by a buildup pattern. Buildup units are similar to pauser units, but lack the initial onset spikes (Rose et $31 .$. 1959; Pfeiffer, 1966; Godfrey et al., 1975a, 1975b: Bourk, 1976).

Studies using intracellular horseradish peroxidase labeling teciniques demonstrated that PI units correspond to spherical bushy ceils, Fir wists to globular bushy cells, choppers to stellate =e:2i, onse: units to multipolar/stellate cells and octopus cells (PVCN; alld pauser-tuildup units to fusiform cells (Rhode et al.. 1983a; 1983b; Rolilier and Ryugo, 1984; Smith and Rhode, 1985; 1987; 19891 .

\section{Cochlear Nucleus Projections to Lemniscal Nuclei}

The CN projections to the NLL (Figure 1) are from stellate/multipolar cells in VCN, octopus cells of PVCN, and giant and fusiform cells in the DCN. The sellate/multipolar cells innervate periolivary nuclei of soc bilaterally but bypass the major binaural nuclei of the soc, then proceed via the contralateral lateral 
lemniscus, and collateralize in all three nuclei of the lateral

lemniscus, the VNLL. INLL and DNLL. These fibers then terminate

tonotopically in the inferior colliculus. The multipolar/stellate

cells also have minor projections to ipsilateral VNLL and IC. The octopus cells of PVCN project to the periolivary groups of the SOC bilaterally, and to the contralateral VNLL; octopus cells do not appear to project to INLL (zook and Casseday, 1979).

Most of the axons from the fusiform and giant cells of the DCN bypass the SOC, cross to the opposite side and project tonotopically to the contralateral inferior colliculus through the lateral lemniscus. As the fibers pass through the lateral lemniscus, they give off collaterals to VNLL (Browner and Webster, 1975; Brunso-Bechtold et al.. 1981: Casseday and Covey, 1987, Coleman and Cierici, 1987; Elverland, 1978; Glendenning et al., 1981; Glendenning and Masterton, 1983; Goldberg and Moore, 1967; Kudo, 1981; Nordeen et al., 1983; Oliver, 1984, 1987; Osen, 1972; Roth et al., 1978; Schweizer, 1981; Tanaka et a... Ls̄5; Warr, 1966; 1969; 1972a; Willard ard Martin, 1983; 200k and Casseday, 1987 ).

Superior Olivary Complex (soc)

The superior olivary complex (SOC), in the lateral ventral pons, is the first nucleus in the central auditory system to receive input from both ears and is thus strategically located in the brainstem for processing binaural time and intensity cues. The three major nuclei of the SOC are the lateral superior olive (LSO), the medial superior olive (MSO), and the medial nucleus of the trapezoid body (MNTB) which lies within the fibers of the trapezoid body. Ventral to the MNTB and Iso, 
respectively, are the ventral and lateral nuclei of the trapezoid bedy (VNTB and LNTB). Scattered and poorly defined groups of neurons at the margins of the soc comprise the periolivary region and include the dorsomedial periolivary nucleus (DMPO), the ventromedial periolivary nucleus (VMPO), dorsolateral periolivary nucleus (DLPO), and the posterior periolivary nucleus (POP).

Comparative studies in mamals have revealed interspecies differences in the relative sizes of soc nuclei (Harrison and Feldman, 1970; Harrison and Irving, 1966a; Irving and harrison, 1967; Moore and Moore, 1971). Mamals with high fregency hearing (e.g. rat, mouse. hedgetog or dolphin) have a relatively small (or absent) MSO, and a large MNTB/LSO; in contrast, MSO is large and MNTB/LSO small or absent in some primates (e.g. humans) which do not possess high frequency hearing, and all three nuclei are developed in the largest group of mammals (e.g. cat, dog, chinchilla, guinea p:g) (Harrison and Howe, 1974: IIving and harrison, 1967; Moore and Moore, 1971). It has been proposea tnat the relative sizes of LSO/MNTB and MSO reflect the relative dependence of the species on high frequency and low frequency cues (interaural intensity differences, IID and interaural phase differences, IPD respectively) for auditory azimuthal localization: physiological evidence offers some support for the hypothesis that the MSO processes IPDs, while the LSO processes IIDs Masterton, 1974: Masterton and Diamond, 1967; Masterton et al., 1975, 2vorykin, 1964). The relative sizes of MNB/LSO and MSO are important in comparing the binaural responses in DNLL for different species. Projections from MSO to DNIL might contribute summation cell (EE cells) responses to DNLL, while projections from LSO might supply suppression cell (IE 
cells) responses. In species with high frequency hearing (e.g. bats, Iat, and little or no MSO, few EE cells would be expected in DNLL, while in cats, which possess both MSO and LSO, both EE and EI cells would be expected.

Lateral superior olive (LSO)

The 250 (in the cat) is made up of several neuronal types, the most prevalent of which is the fusiform cell or principal cell (Helfert and Schwartz, 1986; 1987). Most Iso neurons, except for neurons with CF below $1 \mathrm{kHz}$, are binaurally sensitive suppression cells (Boudreau and Tsuchitani, 1968, 1970; Caird and Klinke, 1983; Goldberg et al.. 1963; Guinan et al.. 1972a, 1972b; Tsuchitani, 1977). These neurons of the LSO respond with an increased firing rate to acoustic stimulation of the ipsilateral ear, an increase that can be inhibited by simultaneous stimulation of the contralateral ear (IE). Thus these neirons show sersitivity to interaural intensity differences (IIDs). The remeining wbo ceils may be monaliral cells only resfonsive to sound fruin irie ifsilateral ear (OE/O cells) or summation (EE/E) cells resconsive to stamulation of either ear (Guinan et al., 1972a, b; Tsuchitani, 1977; Brownell et al., 1979). Most neurons with CF below 1 kla a are monaural OE/O cells (Boudreau and Tsuchitani, 1968; Guinan et al.. 1972a, 1972b). The LSO is tonotopically organized with high frequencies represented ventromedially, and low frequencies dorsolateraliy; there is a disproportionately large representation of high frequencies (Boudreau and Tsuchitani, 1970; Guinan et al., 1972b, Tsuchitani, 1977; Tsuchitani and Boudreau, 1966).

The major sources of afferents to ISO are ipsilateral AVCN and 
MNTB, with over 958 of LSO cells labeled after HRP injections in these nuclei (Glendenning et al., 1985; Cant and Casseday, 1986). Electron microscope studies have identified type 1 synaptic terminals (asymmetric synapses, spherical vesicles), which are presumably excitatory, on the distal dendrites of LSO neurons; and type 2 terminals (symetric synapses, flattened vesicles), presumably inhibitory, on the soma and proximal dendrites of Lso neurons (Wenthold et al., 1987; Glendenning and Baker, 1988; Sanes et al., 1987). Thus it was proposed (Cant, 1984) that the type 1 excitatory terminals result from the projection from ipsilateral AVCN, while the type 2 terminals may constitute the inhibitory glycinergic projection from ipsilateral MNB, providing for a possible neural substrate for the IE cells of LSO. The projection to LSO from AVCN arises from the spherical bushy cells (AA, AP, APD subdivisions of anterior AVCr), and other cells from posterodorsal (PD) division (multipolar, globular bushy or stellate cells): however, the type 1 excitatory terminals on Iso neurons probably arise from the spherical bushy celis (Cant ard Casseday, 1986). The LSo projects bilateraliy to the inferlor colliculus (IC) (cat: Adams, 1979; Brunso-Bechtold et al., 1981; Roth et ai., 1978; Stotler, 1953; rat: Beyerl, 1978; Coleman and Clerica. 1987; opossum: Willard and Martin, 1983; kangaroo rat: Browner ard Webster, 1975; bat: 200k and Casseday, 1982), with 1psilaterally projecting cells concentrated in the lateral limb of the Lso, and contralaterally projecting cells concentrated in the medial $11 \mathrm{mb}$ (Glendenning and Masterton, 1983). Less than 5* of LSO zells project bilaterally, with axon collaterals arising flom the same cell (Glendenning and Masterton, 1983). Anterograde tracer studies showed 
that neurons in the lateral limb of the Lso distribute projections over larger areas in the contralateral rather than ipsilateral IC, while neurons in the medial limb of LSo project to larger areas in the ipsilateral IC (Schneiderman and Henkel, 1987). However, retrograde labelling studies showed projections of the lso to the ipsilateral and contralateral IC more uniformly distribu ed within Iso (rat: Beyerl. 1978).

The bilateral projection of iso to inferior colliculus is paralleled by a bilateral projection of the Iso to the dorsal nucleus of the lateral lemniscus (Elverland, 1978; Glenddening et al.. 1981). Many LSO neuzons are probably GABAergic and thus have inhibitory projections: glutamate decarboxylase (GAD) positive cell bodies are found throughout LSO (rat: Moore and Moore, 1987; cat: Adams and Mugnaini, 1990; gerbil: Roberts and Ribak, 1987), and GABA-positive cell bodies have been reported (guinea pig: Helfert et al.. 1989; Peyret $t$ al.. 1987; Thompson et al., 1985; cat: Adams and Mugnaini, 1sul: Salst-Marie ex al., 1989). That some ISO neurors are giycinergic has been showi. by high levels of tritiated strychnine binding to Lso demanstrated autoradiographically (Giendenning and Baker, 1988; Zarbin tt al., 1991), intense glycine receptor imnuncreactivity in LSO (A)tschuier et al., 1986), and glycine-immunoreactivity of many cell bodies in LSO (Wenthold et al., 1987). High levels of choline acetyltransferase (ChAT) enzymatic activity (Wenthold and Morest, 19761, and presence of acetylcholinesterase containing cells (Osen et al., 1984), may give evidence for cholinergic cells in ISO. 
Medial Superior Olive (MSO)

The Mso consists of a very thin cell plate (estimated 4-5 fusiform cells thick), with horizontal layers of cells with dendrites oriented perp ndicular to the cell plate. Because of the thinness of the cell plate, and because of the presence of a large-amplitude slow potential encountered by an electrode passing through the MSO, single units in the MSO of the cat are extremely difficult to isolate; only a few studies have obtained unit recordings from the MSO.

The sparse data on MSO indicate that approximately two thirds of neurons are EE sumnation cells lat: Guinan et al. 1972a, 1972b; beagle: Goldberg and Brown, 1968). These neurons respond to acoustic stimulation of either ear with an increased firing rate but respond most vigozously when binaural inputs arrive at the ears with a time delay characteristic for each cell. Nonoptimal delays may result in firing rates below that caused by stimulation of either ear, suggesting that inhibition also plays a role in the function of the MSO (Goliburg

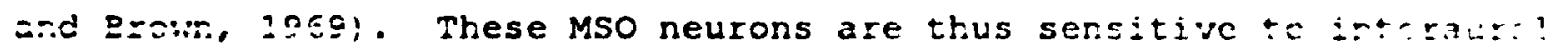
time differences (ITDS), and are termed EE cells since stimulatict, ut either ear has an excitatory effect (although these EE neurons must be distinguished from EE neurons which also may be excited by stimulation of either ear, but are not sensitive to interaural time differences). The remaining cells in the beagle MSO (Goldberg and Brown, 1968) were elther binalial suppression cells (24't EI or IE) or monaural cells lli* EO/O). A study in the rat (Inbody and Feng, 1981) reported larger proportions of binaurally sensitive cells (52: EE, 48 : EI). Sumation (EE' MSO neurons have sharp, V-shaped excitatory turing curves, similar characteristic frequencies (CF) and similar response 
areas for stimulation of the two ears: suppression cells (EI) also had similar CFs for the two ears (Guinan et al., 1972a, 1972b; Goldberg and Brown, 1969; Inbody and Feng, 1981). The MSO is tonotopically organized, with CFs arranged from low to high in the dorsomedial to ventrolateral direction (cat: Guinan et al., 1972b; dog: Goldberg and Brown, 1968; bat: Harnischfeger et al., 1985), following the topographical organization of projections from rostral AVCN to MSO (Van Noort, 1969; Warr, 1966, 1982).

Many neurons in MSO may be glutamatergic (excitatory amino acid) (Godfrey et al.. 1988). GABA does not seem to be an important neurotransmitter in MSO, as glutamate decarboxylase (GAD) positive cell bodies were not found in the MSO in the rat or geribil, but a few GABA. immunoreactive cells were reported in the guinea pig (Helfert et al.. 1989; Peyret et al., 1987; Thompson et al., 1985).

Medial Nucleus of the Trapezoid Body (MNTB) saseu upon electron microscope and Golgi studies, the MVIE has three types of neurons: principal, elongate and stellate neurons (Morest, 1968a, 1968b; Jean-Baptiste and Morest, 1975). Each principal neuron receives one large axosomatic calyx of Held arising from largediameter trapezoid body axons, and each calyx ends on onjy one principal neuron (Morest, 1968a, 1968b). These calyces are among the largest synaptic terminals in the mammalian brain and presumably afford secure synaptic transmission and short response latencies compared to cells in other SOC nuclei (Goldberg and Brown, 1968; Guinan et al., $1972 a, 1972 b)$. Degeneration of large-diameter trapezoid body axons and calyces in the contralateral MarB after lesions limited to the globular 
cell region of CN (PV division of posterior AVCN) (Van Noort, 1969; Narr, 1972a, 1982; Harrison and Irving, 1966), suggest these terminals arise from globular cells in AVCN.

Most MNB neurons (65 of a sample of 218 ) (Guinan et al.. 1972a, 1972b) have a complex waveform, with a positive prepotential reflecting presynaptic activity from the calyces of Held (Li and Guinan, 1971). All neurons with complex waveforms, and the majority of the other units in MNTB were monaural cells only responsive to contralateral srund stimulation (EO/O cells) (Guinan et al., 1972a, 1972b). As expected on the basis of afferent projections, MNB principal neurons have response properties resembling those of the large diameter trapezoid body fibers (Brownel1, 1975), and those of globular bushy cells in posterior AvCN. Thus, principal neurons have primarylike with notch, primarylike or phase-locked PSTHs, irregular firing patterns, and narrow V-shaped tuning curves (Guinan et al. 1972a, 1972b).

Erincipel neurons are tonotopically organized, with high to low ch:aracteristu: frequency organized in a ventromedial to dorsolateral difection (Guinan et al., 1972a, 1972b). Since cell density is lower in the dorsolateral part of the MNTB, there is a disproportionately large represertation of high fregencies in MNTB (Guinan et al.. 1972a, 19720).

Neurons without the complex waveform (possibly stellate reurons) did not show evidence of tonotopic organization, and showed more diverse response properties, including broad tuning curves, and responses at stimulus offset (Guinan et al., 1972b).

Anterograde degeneration studies showed that the cells of MuTB project to the LSO and dorsomedial periolivary nucleus (IMPO) of the 
same side (cat: Van Noort, 1969; Rasmussen, 1967; rabbit: Borg, 1973; kangaroo rat: Browner and Webster, 1975), and give off collaterals to the MSO (Spangler et al., 1985). Anterograde tracer studies show a dense tonotopically organized projection from MarT to all parts of LSO (cat: Elverland, 1978; Glendenning et al., 1985). Injections of horseradish peroxidase in LSO labeled many neurons in ipsilateral MNB, most of which were principal cells (Glendenning et al., 1985). The one-to-one bushy cell (AVCN) to principal cell (MNTB) projection, and the secure synaptic transmission afforded by the large calyx terminals, has lead to suggestions that MNTB serves as a relay between posterior AVCN and LSO (Goldberg and Brown, 1968); since MNTB probably supplies the glycinergic (inhibitory) terminals on LSO neurons (Cant and Casseday, 1986; Spangler et al., 1985) it seems to function as a segregated nucleus of inhibitory interneurons supplying the inhibition in IE cells in LSO.

The MNB also has projections to ipsilateral INLL and VNLL Giencerring $\in$ al., 1981 ) (Figure 3 ). 
Figure 3. Schematic diagrams summarizing ascending projections to the nuclei of the lateral lemniscus from ipsilateral and contralateral (shown to ieft of broken vertical line) subdivisions of (A) the cochlear nucleus and (B) the superior clivary complex. Major and minor profections are shown by tull and broken lines, respectively. crosshatched area in ventral nucleus of the lateral lemniscus (VNLL) indicates dorsomedial region receiving input from ipsilateral ventral cochlear nucleus (VCN). Although all projections have been shown is terminating in the lemniscal nuelel. it is uncertain to what extent they are derived from collaterals of fibers proceeding to the inferior colliculus (Adapted from Irvine, 1986).
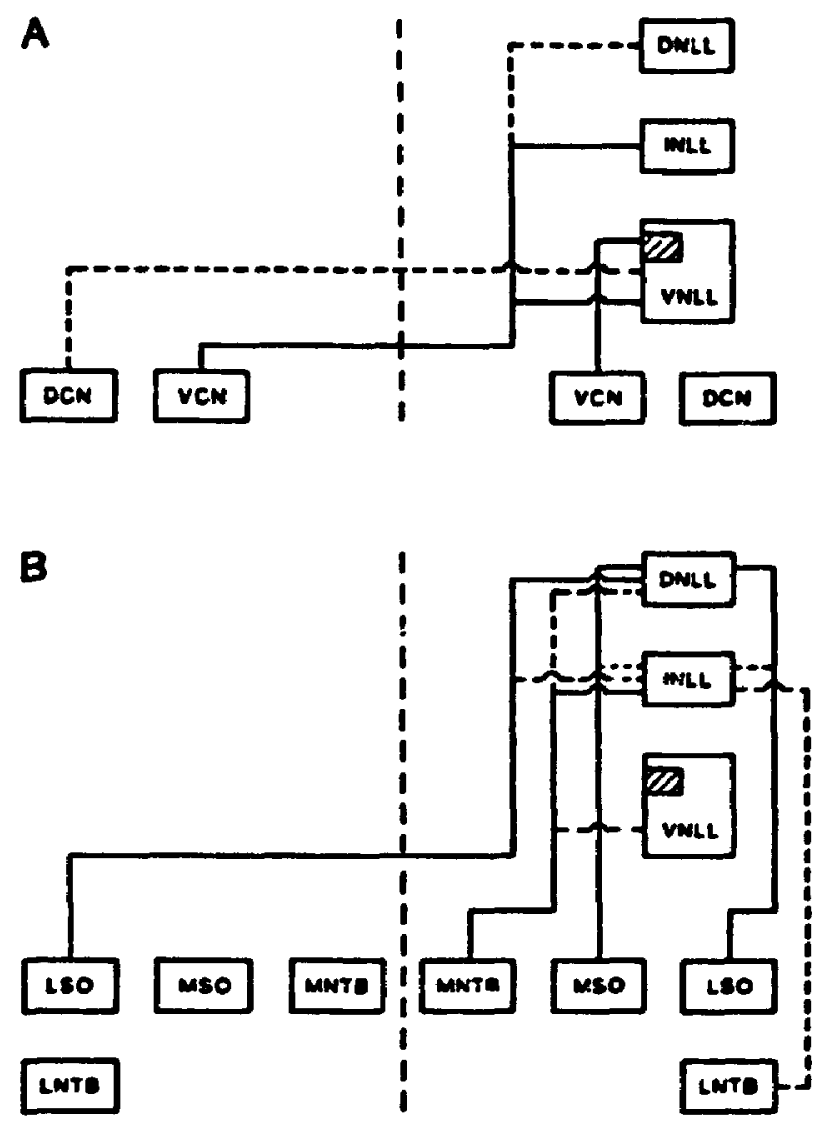
Nuclei of the Lateral Lemniscus

Ascending Projections to Nuclei of Lateral Lemniscus:

The binaural dorsal nucleus of the lateral lemniscus (DNLL) and monaural ventral nucleus of the lateral lemniscus (VNLL) have different patterns of ascending input which may explain their binaural/monaural properties (Figure 3i. The major sources of ascending afferents to DNLL are ipsilateral MSO, and ipsilateral and contralateral LSO (Elverland, 1978; Glendenning and Masterton, 1983; Henkei and Spangler, 1983; Oliver and Shneiderman, 1989; Shneiderman, Oliver and Henkel, 1988; Van Noort, 1969); after HRP injections in DNLL, more than 70 of labeled cells were located in SOC (Glendenning et al., 1981). Smaller projections were derived from contralateral dorsal cochlear nucleus (DCN) (Fernandez and Karpas, 1967; Adams and Warr, 1976; Adams, 1979), contralateral DNLL (Papez, 1929; Goldberg and Moore, 1967; Conlee, 1979; Olıver and Schneiderman, 1989), ipsilateral VNLL (Glendenning et ai., 1531: Harr, 1966, 19821, and from the low frequency part of contralateral anteroventral cochlear nucleus (AVCN) to dorsolateral DNIL (Oliver, Schneiderman and Henkel, 1987). Anterograde tracer studies in the cat showed that the lateral limb of Lso (the lowfrequency partl projects to dorsal DNLL, while the more medial part of LSO (high frequency) projects to ventral DNLL. In ventral DNLL, the projection from LSO showed a mediolateral gradient, with heavier labeling in the medial areas of DNLL on the contralateral side, and heavier labeling in lateral DNLL on the ipsilateral side (Shneiderman, Oliver and Henkel, 1988). Injections in the dorsal (low frequency) part of MSO labeled the dorsal part of DNLL; however, the ventral (high 
frequencyl part of MSO projected only to the middle of DNLL. The projection from AVCN to DNLI was bilateral only for injections in ventrolateral (low frequency) $A V C N$, in which case projections were to dorsal DNLL. Injections in the dorsomedial (high frequency) AVCN labeled only ventral DNLL exclusively on the contralateral side (Shneiderman, Oliver and Henkel, 1988).

Most DNLL neurons contain GABA, a putative inhibitory neurotransmitter (Adams and Mugnaini, 1984; Roberts and Ribak, 1987: Thompson, Cortez and Lam, 1985; Moore and Moore, 1987), and many of the projection neurons of DNLL to IC are GABAergic (Adams and Mugnaini, 1984). Electron microscope studies showed two types of synaptic terminals in roughly equal proportions in DNLL: terminals with round synaptic vesicles and asymmetric junctions (probably excitatory), and terminals with pleiomorphic vesicles and symetric junctions (probably inhibitoryl found on axons from cells in contralateral DNLL. This suggests inhibitory, reciprocal connections between the two DNLL 101iyer and Srneiderman, 1989; Shneiderman and Oliver, 1969).

In contrast to the projections from binaural sources tC DNL:, the major source of ascending afferents to VNLL is contralateral VCN (Glendenning et al., 1981; Van Noort, 1969; Warr, 1966; 1969; 1972a; 1982), with three times as many cells projecting from AVCN as do cells from PVCN (Glendenning et al., 1981). There are also weak projections from contralateral DCN shown by degeneration and tracer studies (Adams and Warr, 1976; Fernandez and Karapas, 1967; Glendenning et al., 1981), and a weak projection from ipsilateral soc (Elverland, 1978; Giendenning et al., 1981; Henkel and Spangler, 1983), but this latter projection appears to be derived from MNB (probably monaural, 
contralaterally sensitive cells) and periolivary cell groups rather than from the major binaural nuclei of the soc (Glendenning and Masterton, 1983; Glendenning et al., 1981). A small dorsomedial region of VNLL receives the only ipsilateral projection, from VCN (cat: Glendenning et al., 1981; Warr, 1969; rhesus monkey: Strominger and Strominger, 1971): except for this projection, we would expect only monaural cells in VNLL, sensitive to only contilateral stimulation. Octopus cells of the PVCN project axons to the VNLL, with heaviest terminal degeneration observed in lateral VNLL (Van Noort, 1969; Warr, 1969; 1982: Adams and Warr, 1976). These axons do not appear to reach the inferior colliculus (IC). In the rat, they send collaterals to the superior olivary complex on both sides before terminating in VNLL (Friauf and Ostwald, 1988). Immunocytochemical and uptake/transport studies have shown that a large number of VNLL neurons are glycinergic and Inhibitory (Adams and Wenthold, 1987; Aoki et al., 1988; Saint Marie and Baker, i990). Terminals immunoreactive for glutamic acid decof uryiase $(G A S+)$ are present in the VNLI (Roberts and Ribak, 1987). The pittern of afferent connections to intermediate nucleus of the lateral lemniscus (INLL) is in some respects intermediate between those to DNLL and VNLL. It receives substantial projections from contralateral VCN (with three times as many cells in AVCN as in PVCN), from ipsilateral MSO and Lso bilaterally, and from ipsilateral VNLL (Glenderning et al., 1981; van Noort, 1969). However, in contrast to DNLL and VNLL, after horseradish peroxidase injections in INIL $208-258$ of labeled cells are located in ipsilateral MNTB (Glendenning et al., 1981). A heavy projection from LNTB to INLL was reported in the bat (200k and Casseday, 1979), but very little (maximum of 18) of the 
projection to INLL is from LNTB in the cat (Glendenning et al.. 1981). Neurons in INLL are immunoreactive for the excitatory neurotransmitter aspartate $(A S P+)$, and are surrounded by terminals immonoreactive for glutamic acid decarboxylase $(G A D+)$ (Roberts and Ribak, 1987).

Cellular Morphology in Leraniscal Nuclei:

Cellular Morphology: DNLL

Only three studies have been devoted to cellular morphology in the NLL, and all of these studies were restricted to the DNLI (cat: Kane and Barone, 1980; rat: Bajo et al., 1993; mouse: Iwahori, 1986), while brief descriptions of neurons in NLL have been given incidentally in a few studies of NLL projections (Adams, 1979; Adams and Mugnaini, 1984; Covey and Casseday, 1986; Glendenning et al., 1981; Taber, 1961; Willard and Ryugo, 1983; Shneiderman et al., 1988; Oliver and Shreiderman, 1989; Hutson et al.. 1991). In a brief account, Adars (1979: describes DNIL as having larg (19-27 micron diameter) cells b:zis aarkiy szaining Nissl substance, and smaller elorigate celis $120-13$ microns): multipolar cells (17-24 microns) which lacked the cap of Nissl substarce around their nuclei were interspersed among the larger cells.

Subsequently, Kane and Barone (1980) differentiated rine types of Nissl-stained DNLL neurons, on the basis of size and shape: (1) large round (LR), (2) large ovoid (LO), (3) large elongate (LE), (4) medium round (MR), (5) medium ovoid (MO), (6) medium elongate (ME), (7) small round (SR), (8) small ovoid (SO), (9) small elongate (SE). The elongate and multipoiar cells of Adams (1979) were demonstrated to be equivalent to LO and LE cells, and LR cells, respectively (Kane and Barone, 1980). 
The majority (84t) of neurons were round or ovoid, and virtually all (99.5\%) were large (cell diameters greater than 22 microns) or medium sized (soma diameters 12-22 microns) (Kane and Barone, 1980). However, the DNLL is surroundzd ventrally, laterally and ventromedially by clustered small cells; the DNLL is nearly continuous dorsally with the ventrolateral division of inferior colliculus, laterally with the nucleus sagulum, and ventrally with smaller neurons in the INLL (Kane and Barone, 19801 .

Progressively more large and round neuron types were found along the caudal-to-rostral axis in DNLL. Caudally, neurons had a more compact appearance and were closely clustered, while rostrally, neurons were more loost $y$ organized, with cell clusters separated by lateral lemniscus fibers; furthermore, ovoid or elongate neurons whose long axis was horizontally oriented were caudally located, while more vertically cells appeared more rostrally. Rostral and caudal divisions may be present in DNLL, which also have differing efferent projectzons (a)scussed below) (Kane and Barone, 1980).

Intermediate-sized cells in Nissl-stained DNII were predominant in the caudal, lateral, and medial parts of the nucleus, surrolinding the more centrally and rostrally located large cells (Kane and Barone, 19801 .

or the basis of dendritic arbor and cytology. oriertation within DNLI, and axonal morphology, Golgi impregnations showed: subdivisions of the LO class into horizontal (LOH, horizontally oriented soma and dendrites) and vertical (LOV, vertically oriented soma and dendrites; less common type) types, and 12 , subdivisions of MR neurons into radiate (MRR, dendrites radiating in all directions) and 
oriented (MRO, dendrites running either medially or laterally in the nucleus) subclasses. No small neurons were found in the Golgi impregnations (Kane and Barone, 1980).

Neurons from all classes of large neurons, especially LE neurons, could have ventrally directed axons, probabiy supplying descending input to cochlear nucleus. All large neuron types, especially Lov, also showed examples of dorsally directed axons which might project to inferior colliculus. LE and LR neurons had dendritic fields positioned so that they could receive inputs from large numbers of lemniscal fibers (both ascending and descending). In contrast to large neurons, medium-sized neurons arborized locally and many may be DNLL interneurons. The MR cells (particularly MRR) had the longest axons, with many axons directed medially, and thus may contribute to the projection to contralateral DNLL (Kane and Barone, 1980).

Nearly all ovoid and elongate somata had a preferential horizontal orientation, with a slight tilt from dorsomedial to ventrolateral, and Goigi impregnations showed that most cell somata and their derdritic trees were preferentially oriented in horizontal planes, roughly perperdicular to the course of the lateral lemniscus (Kane and Barone, $1980)$.

Similar cell types, including large and medium fusiform cells, and cytoarchitecture have been found in the bat DNLL (mustache bat: 200 . and Casseday, 1982; horseshoe bat: Vater and Feng, 19901, and in the mouse (Iwahori, 1986). In the human auditory system, DNLL is similar in size and cytoarchitecture to that of the cat, but the largest cell types are absent (Moore, 1987).

A recent study devoted to cellular morphology of the DNLI in the 
rat (Bajo et al., 1993) found four well-differentiated neuronal types, as seen in Nissl-stained material. The morphological criteria which this classification was based upon included size, shape and contour of cell body, the Nissl pattern of the cytoplasm, the size and position of the nucleus and numleolus, and irregularities in the nuclear envelope. Type I neurons were described as large and multipolar, with abundant cytoplasm and darkly stained Nissl substance. Type II neurons were iarge, bipolar and darkly stained in Nissl material. Type III neurons were medium in size and their soma were round or ovoid. Type IV neurons were small and round with scant cytoplasm, and appeared to be the least common neuronal type in DNLL (Bajo et al., 1993).

It was concluded (Bajo et al., 1993) that type I neurons 12kely correspond to type I neurons found in the mouse with Golgi technique (Iwahori, 1986), to large round and/or ovoid neurons described in Nissl material in the cat (Kane and Barone, 1980), and to the large spherical cells found to predominate in the dorsal part of the nucleus in the cat (Shuedielman $\in t$ al., 1988). Type II neurons found in the rat (Baj= et a... 1993) likely corresponded to types II and/or II neurons in the mouse (Iwahori, 1966), to the large elongate neurons in the cat (Kane and Barone, 1980), or to fusiform cells (cat: Shneiderman et al., 1988). Type III neurons (Bajo et al., 1993) probably corresponded to medium-sized neurons in the cat (Kane and Barone, 1980), or to small rounded cells located mainly in the lateral part of the cat DNLL described by Shneiderman et al. (1988). Type IV neurons resembled small DNLI neurons described by Adams and Mugnaini (1984) in the cat (Bajo et al.. 1993).

The four neuronal types in the rat differed somewhat in their 
spatial distribution within DNLL (Bajo et al., 1993). Type I neurons were preferentially distributed in the most dorsal and caudal parts of DNLL; type II neurons were intermingled with the fibers entering and exiting the DNLL via the commissure of Probst land projecting to the DNLL on the opposite side) (Bajo et al., 1993). Type III neurons were more homogeneously distributed, although highest numbers of these neurons were found in lateral DNLL; type IV neurons were scarce, but found throughout DNLL (Bajo et al., 1993).

\section{Cellular Morphology: INLL}

Very little information is available on the cellular morfhology 15. the INLL, except for incidental observations reported in studies of other structures (Glendenning et al., 1981; Adams, 1979; 20ok and Casseday, 1982b; 1979; Covey and Casseday, 1986; Vater and Feng, 19901. In Nissl material, cells in INLL were sparsely distrubuted and preciominantly muitipolar (12-25 microns) in shape, resembling the rustifuid: weids in DNLL in that they had finely dispersed : substance and frequently lacked a cap of Nissl substance around the. I nuclei (Adams, 1979; Glendenning et al., 1981). Clusters of horzzontal cells were also present (Glendenning et al., 1981). In Golgl preparations in the mouse, INLL neurons had horizontally oriented dendrites like those in DNLL (mouse: Willard and Ryugo, 19831 , but no Golgl study has yet been done in the cat. No separate INLL region is differentiated in the human auditory system (Moore, 1987).

A small amount of information is also available on INLL in the bat, where the structure is hypertrophied (Schweizer, 1981; 2ock and Casseday, 1979; 1982b; Covey and Casseday, 1986), and could have a 
substantially different morphology than that in the cat or mouse. In the mustache bat, most neurons were elongate cells with dendrites oriented mediolaterally and parallel to fibers that course horizontally from the lateral lemiscus. Large globular cells with eccentrically placed nuclei, as well as small and medium-sized ovoid cells were also present (200k and Casseday, 1982b). At the dorsal tip of the INLL, there was an increase in cell packing density, and an increase in the number of large globular cells (Zook and Casseday, 1982b). The cells in INLI showed little resemblance to those in DNLL or VNLL (zook and Casseday, 1979; 1982b). In the hozeshoe bat, INLI was found to contain ovoid cells arranged in vertical columns between the fibers of the lateral lemniscus, and had poorly defined medial and dorsal borders (Vater and Feng, 1990$)$.

In the brown bat, E. fuscus, INIL contained a mixture of cell types, with most ceils elongate or globular, with dendrites oriented orthoonally to the ascending lateral lemiscus fibers (Covey and rasssiay, 1986). A few multipolar cells were alsc found. The cells were tess densely packed in INLL than they were in VNiL, and there was no columnar organization. At the ventral border of INLL, there was a transitional area containing medium-sized globular, multipolar and elongate cells that have a columnar organization anteriorly (Covey and Cr eday. 1986).

\section{Cellular Morphology: VNLL}

In the cat and mouse, VNLL is subdivided into medial and ventral (called "lateral" in the mouse) divisions (Cat: Adams, 1979; Glendenning et al., 1981; mouse: Willard and Ryugo, 1983). Most cells 
found in the medial zone in both species were medium and large multipolar cells (12-20 microns) and horizontal cells (9-11 microns) with dendrites perpendicular to the ascending auditory fibers: small cells (6-12 microns) were also present. Most cells had a distinct cap of Nissl substance around their nuclei (Adams, 1979; Glendenning et al., 1981).

The most common cell type found in the ventral zone of VNLL was an oval-shaped, globular neuron (10-20 microns) resembling the principal celis of MNTB and was seen to receive large axosomatic calyceal endings similar to those in MNTB, in protargol material (Adams, 1979; Glendenring et al.. 1981; van Noort, 1969; Stotler, 1953; mouse: Willard and Ryugo, 1983). Elongate cells, and small cells also were found (Adams, 1979).

In the echolocating bat (E. fuscus), VILL is divided into a dorsal columnar area (VNLLC) with small round or oval cells tightly packed togethe: in columns parallel to the ascending lateral lemniscus fibers, and a ventral multipolar area (VNLLm) of multipolar neurons with izregularly staped somata with multiple large dendrites (Covey and Casseday, 1985; 1991). There were also a few globular and elongate cells present in the WILm division.

Although a columnar area was also seen in the mustache bat, it was found in the most ventral part of VNLL (Zook and Casseday, 1982b). The vertral columnar area consisted exclusively of small, multipolar cells densely packeo in colunns. The dorsal part of VNLL also contained smali multipolar cells, but with a great variety of shapes, which included elongete cells with their long axes oriented at right angles to the lateral lemniscus tract (200k and Casseday, 1982b). 
In the horseshoe bat, a lateral zone of VNLL (corresponding to the dorsal division in the cat) had a heterogeneous cell population, consisting of multipolar, elongate and round cells; the medial zone had a high packing density of round cells (Vater and Feng, 1990).

\section{Physiological Responses of Nuclei of Lateral Lemniscus:}

Dorsal Nucleus of the Lateral Lemniscus:

The handful of studies of the physiological response properties of NLL show a contrast between the binaural sensitivity of DNLL neurons, and the monaural sensitivity of INLL and VNLL neurons (Aitkin et al., 1970; Brugge et al., 1970; Guinan et al., 1972a, 1972b; Covey and Casseday, 1991: Covey, 1993), which could be expected on the basis of afferent connections to NLL. In accordance with the projections to DNLI from binaurally sensitive nuclei of the SOC, most DNLL neurons in the cat had binaural response properties (88z of a sample of 121), which included a majority of suppression (EI) responses (cat: Aitkin eif.. 1970). The remaining 128 of neurons were monairal, sat influenced only by stimulation of the contralateral ear $(11 / 12 i$ urits were EC/O) or ipsilateral ear (3/121 units were OE/O).

The dorsal part of DNLL contained cells responsive to low frequencies and sensitive to variations in interaural time difference (ITD): the delay that produced maximal neuronal discharge was that which brought the monaural discharges into coincidence, as was found previously in MSO neurons (Goldberg and Brown, 1969), which project to dorsal DNLL. Unlike MSO neurons, however, some of these neurors appeared to have EO/I suppression responses for binaural stimulation with no interaural time difference. Some EE cells showed onset 
reponses to monaural stimulation, but only showed a sustained reponse with an optimal delay between ipsilateral and contralateral stimulation (Brugge et al., 1970).

More ventrally in DNLI, the neurons responded to higher frequencies and were usually inhibited by ipsilateral stimulation and excited by contralateral stimulation (EO/I cells) and were influenced by changes in the interaural intensity difference (IID). These neurons were not sensitive to interaural time differences (Aitkin et al.. 1970; Brugge et al.. 1970). These neurons had IID functions similar to those found for EO/I cells in MSO or for OE/I cells in LSO (Goldberg and Brown. 1969).

Similarity of binaural responding in DNLL to that in MSO and LSO suggests that these soc nuclei may be the source of the binaural interactions in DNLI, but does not rule out the possibility that binaural interactions are generated within DNIL. The conspicuous lack of binaural IE cells in DNLL lexcited by ipsilateral stimulation. ishitited with simultaneous contralateral stimulation), which might have been expected from the projection of IE cells from ipsilateral Lso, is paralleled by a lack of such cells in inferior colliculus (IC), which also receives an ipsilateral LSO projection. The lack of IE cells in DNLL or IC may be explained by evidence that most projections from ipsilateral Iso are glycinergic and therefore probably inhibitory (Saint-Marie et al.. 1989; Saint-Marie and Baker, 1990; Hutson et al., 1987). Binaural sensitivity in DNLI allowing detection of ITDs of IIDs suggests a role for DNLL in sound localization (Aitrin et al., 1970; Brugge et al., 1970).

Response patterns for DNLL neurons included onset lone or more 
spikes), onset-pause-sustained, or sustained responses, with irregular firing patterns not reflecting the chopper patterns of presumed inputs from stellate cells in VCN. The response patterns of some neurons changed with frequency and intensity of sound stimulation, suggesting an interaction between excitatory and inhibitory sources (Brugge et al., 1970). Some neurons showed phase-locking at frequencies lower than $1 \mathrm{kHz}$ (Aitkin et al., 1970; Brugge et al., 1970). In some EO/I cells sensitive to IIDs, the onset spikes of the sustained resfonses were never suppressed, even at large IIDs favouring the ipsilateral ear. All neurons had sharp frequency tuning, but some showed inhibition of spontaneous activity when they were stimulated with frequencies other than the characteristic frequency (i.e., outside of theif excitatory response region) (Aitkin et al., 1970; Brugge et al., $1970 \%$

In the bat, E. fuscus (Covey and Casseday, 1991), all neurons in DNLL were found to have EO/I suppression responses. A more recent stucy (Covey, 1593) of DNLL and the lateral tegmental area just mesial to DNLL (parajemniscal zone), and of the rostral part of DNLL (termed dorsal paralemniscal zone in this study) in the same species of bat, found a predominance of EO/I suppression responses in DNLL, but other bدnaural response types were present (518 EO/I; $10 £ \mathrm{EO} / \mathrm{I}$ mixed; 28 EO/F; 12 EE; 78 EO/O; 28 OI/O; sample of 49). In total, 648 of units in DNLL were binaurally sensitive. Binaural facilitation, in which the response of neurons was greatly enhanced over a certain range of interaural intensity differences, occurred in 12 of the units in DNLL (in EO/F, or EE/F neurons). Little evidence of an orderly tonotopic progression of characteristic frequencies in DNLL was found; instead, 
neurons with similar characteristic frequency were clustered together in patches (mosaic arrangement), and lower frequencies tended to be represented more dorsally than higher frequencies (Covey, 1993). Rostral DNLL (dorsal paralemniscal zone, DPL) was distinguished from the main division of DNLL, since it had only 298 binaural sensitivity (298 EO/I; 718 EO/O), which did not include any EE sumnation responses or binaural facilitation The majority of neurons in DPL had onset responses, in contrast to the majority of sustained responses found in DNLI. A proportion of neurons in both DNLL and DPL were choppers (12* in DNLL; 38 in DRL).

Whether or not there is an orderly tonotopic representation within DNLL and what form this representation may take remains controversial. While studies in the bat (Covey and Casseday, 1991; Covey, 1993) and cat (Aitkin et al., 1970) have found some evidence of a progression of frequencies with low frequencies represented dorsally, and higher frequencies nore ventrally, other studies employing uptake of $14 \mathrm{C}-2$. decy:gluesse sollowing low or high frequency stimulation (cat: Nurdo and Maszerion, 1984) failed to find evidence of tonotopy in DNLL.

In a c-fos study after pure-tone acoustic stimulation, an orderly tonotopic organization was found: higher frequencies were found to be represented ventrally and dorsally within DNLL, while lower frequencies were represented centrally within DNLI (Friauf, 1992). These findings were flirther corroborated by results in the same lab (Preuß, 1991); injections of two retrograde fluorescent tracers into electrophysiologically characterized sites in the central nucleus of the IC, shoived the same mirror-symmetric arrangement of labeled nesrors in the DNLI. 
Another study which studied descending projections from the central nucleus of IC to the DNLL using the anterograde axonal tracer Phaseolis vulgaris-leucoagglutinin (PHA-L) found that injections from sites in the IC resulted in mediolaterally oriented bands of terminal labeling. found dorsally and ventrally within DNLL. A shift of injection sites along the dorsoventral axis of ICC (to higher frequencies) resulted in a shift in labeled terminal bands within DNLL towards the dorsal and ventral boundaries of DNLL (Caicedo and Herbert, 1993). Thus the descending inputs from the IC to DNLL afpear to have a tonotopic arrangement which would be compatible with the results of Friauf (1992) and Preuß (1991).

Another study, in the rat and cat (Saldana, 1993) has found using retrograde and anterograde tracers, a ring-like pattern of terminals labeled in DNLL following small injections into the ICC. As higher frequency sites in 30 were injected, the labeled ring in DNLI. shifted outwards, towards the outer borders of DNLL. For lower frequency irjecticris in IC, the central part of DNLL was labeled. Cis this basis, ar. "orion-like" organization of DNLL was proposed, in which low frequencies were represented centrally within DNLL, and higher frequencies were represented in surrounding outer rings (Saldana, 1993). In this model, the position of the labeled rings was found to vary along the rostral-caudal axis, resulting in a very complicated model of frequency representation. In addition, not all injections in IC resulted in complete labeied rings, and in many cases the numbers of labeled neurons on parts of the rings were very smali. At many levels within DNLI, the labeled rings were incomplete laterally and medially, allowing for a possibility that the results of saldana (1993), Friauf 
(1992), and Preuß (1991) might be reconciled.

Ventral and Intermediate Nuclei of the Lateral Lemniscus:

In contrast to the binaural sensitivity of DNLL neurons, most VNLL units (898 of a sample of 57$)$ were monaural and responded only to contralateral stimulation (EO/O), while only 118 were binaurally sensitive (cat: Aitkin et al., 1970), which accords with contralateral monaural cells of VCN supplying the major afferent input to VNLL. The few binaural units were located near the dorsal and ventral margins of VNLL and were not intermingled with the EO/O colls. Like the DNLI, the VNLL is tonotopically organized with highest characteristic frequencies found ventrally, and lowest frequencies dorsally. The response patterns of VNLL neurons were similar to those found for DNLL neurons, and some VNLL neurons also showed phase locking at frequencies be' ow 1 kHz. Two VNLL neurons were found in which the number of spikes discharged during the tone was a non-monotonic function of intensity (A.jik_r et ai., 1970; Brugge et al., 1970).

A second study of 32 VNLL neurons in the cat /Guinan et al., 1972a, $1572 b)$ differed from the first in finding no tonotopic organization in. VNLL, and in reporting 37.5 monaural (EO/O), and 56.7 binaural (EE or EI) neurons, with the two response types intermingled. Most units had sharp frequency tuning curves, but a few units were broadly tuned or had departures from a V-shaped tuning curve (Guinan et al., 1972a, $1972 b)$.

Neurons in INLL had properties intermediate between those of DNLL and WNLL, being partly binaural (62z of a sample of 37 ) and partly monaural and only responsive to contralateral stimulation $(38 \%)$; no 
tonotopic organization was apparent lAitkin et al.. 1969; Brugge et a1.. 1970).

In the echolocating bat. E. fuscus, in which the two subdivisions of VNLL are the dorsal coiumar area (VNLLC), and the ventral multipolar area (VNLLm), injections of tracers in AVCN or IC at sites where the characteristic frequency of cells had been determined electrophysiologically, demonstrated the precise frequency organization of projections to VNLLc (Covey and Casseday, 1986). A less precise tonotopic organization was found in INLL and VNIIm; however, each subdivision had a complete frequency representation (Covey and Casseday, 1986). Earlier studies in the cat may have missed the tonotopic organization in VNLL because of the presence of two subdivisions. An overrepresentation of frequencies $(25-50 \mathrm{kHz})$ corresponding to the range of the FM echolocation call of the bat was found in VNLLC (Covey and Casseday, 1986).

Tonotopic organization in VNLL was suggested by differential aorsal of vertial uptake of 14c-2-deoxyglucose following low or tizgis irequericy stimulation, respectively (gerbil: Ryan et al., 1982), but other studies icat: Nudo and Masterton, 1984) failed to find evidence of tonotopy in VNLL or DNLI.

Virtually all cells in INLL, VNLLm and VNLLc were monaural (EO/O), responding only to contralateral stimulation; a region medial to INLI and VNLLc, not part of the conventional divisions of NLI, had binaural EO/I celis (Covey and Casseday, 1991). However, no testing of sensizivity to ITDs seems to have been made in this study (Covey and Casseday, 1991). Units in INLL, VNLLc and VNLLm all had broad tuning to frequency. little or no spontaneous activity and short integration 
times (responding to stimuli less than 5 msec in duration), making these neurons potentially important in encoding temporal information. Neurons in VNLLC were the most broadly tuned and responded with a single spike precisely locked to stimulus onset over large variations in frequency or intensity; in INLL and VNLLm, response patterns were evenly distributed between tonic (discharge at a constant high rate throughout the duration of the stimulus), choppers and onset (Covey and Casseday, 1991). The presence of choppers and onset responses is in accordance with projections from contralateral VCN loctopus and stellate cells). The response characteristics of these subdivisions lead to the suggestion that VNLLc encodes stimuius onset, while INLL and VNLLm encode ongoing temporal properties of a sound lCovey and Casseday, 19911.

Ascending Projections From Nuclei of Lateral Lemniscus:

Binaural DNLL and the monaural nuclei, VNLL and INLL, may also be distinguished on the basis of different ascending projections to the auditory midbrain. DNLL has strong projections to contralateral DNLL (Kudo, 1981) and the ipsilateral and contralateral central nucleus of the inferior colliculus (ICC) while VNLL projects almost exclusively to ipsilateral ICC and DNLL (Woollard and Harpman, 1940; Shneiderman, O:iver and Henkel, 1988; Moore, 1987; Adams, 1979; Brunso-Bechtold et al., 1981; Kudo, 1981; Roth et al.. 1978; Elverland, 1978; Adams and Wenthold, 1987 Goldberg and Moore, 1967; Bajo et al., 1993). Projettions from lemniscal nuclei to inferior colliculus may be classified on the basis of whether projections are focused (terminating 
in discrete terminal fields or bands relating to tonotopic organization) or diffuse (terminating widely within one or more subdivisions of IC). Focused projections from NLL to IC are from contralateral DNLL, while diffuse projections are from ipsilateral DNLL, and ipsilateral INLL and VNLL, suggesting some basic functional difference between the contralateral DNLI projection and the other projections to IC (Casseday and Covey, 1987; Covey and Casseday, 1986; Oliver and Shneiderman, 1989; Schweizer, 1981: Shneiderman and Oli =r, 1989; Tanaka et al.. 1985; 200k and Casseday, 2987; Bajo et al.. 19931. Cells in caudal DNLL project to IC, with those projecting ipsilaterally located centrally, and those projecting to contralaterally located medially and laterally; some neurons project to both ipsilateral and contralateral IC (Tanaka et al., 1985). Rostral DNLL probably projects to the deep layers of superior colliculus (Tanaka et al., 1985; Kudo, 1981; Bajo et al.., 1993), although a recent study failed to find such a projection in the cat (Hutson et al.. is5i,. In the superzor colliculus deep layers, there is a maf of auditory space, based on neurons that respond to auditory as well as visual stimulation; neurons show broad frequency tuning and respond to many auditory or visual stimuli. However, the response of neurons changes depending on the spatial location of the stimulus: most neurons show EO/I suppression responses for auditory stimuli (Allon and Wollberg, 1978; Chalupa and Rhoades, 1977; Mast and Chung, 1973; Wise and Irvine, 1983). Stimulation of neurons in deep superior colliculus appears to produce ear and neck movements (Henkel and Edwards, 1978; Schaefer, 1970; Thompson and Masterton, 1978). The finding of differing projections of rostral and caudal DNLL has lead to the 
suggestion that DNLL divides the auditory information for the acoustic motor reflex from that intended for further processing in the inferior colliculus and medial geniculate body (Tanaka et al.. 1985).

In the cat, the INLL has been reported to project bilaterally to ICC (Brunso-Bechtold et al., 1981); however, in the bat INLL projects only to ipsilateral ICC, to the same region of ICC as the VNLL Izook and Casseday, 1982; Covey and Casseday, 1986).

The NLL also have ascending projections that bypass the ICC: both the DNLL and VNLL project to the medial and dorsal divisions of the medial geniculate body (Henkel, 1983; Kudo, 1981; Whitley and Henkel, 1984; Bajo et al., 19931. Projections of DNLL to superior colliculus (Kudo, 1981; Tanaka et al.. 1985), and NLL to the midbrain reticular formation (Irvine and Jackson, 1983; Stotlex, 1953; Davis et al., 1982) also bypass the IC.

\section{Eossikie Role of the DNLL: Sound Localization}

Zite jilil is binaurally sensitive, and is the first nucleus ir. ste auditsy system to receive projections from both the major binaural superior olive nuclei, the LSO and MSO; thus the DNLL may be in an icieal position for processing binaural cues in sound localization, based on some weighting of the inputs from LSO and MSO.

If the DNLL is involvet in binaural response patterns, then this must be reconciled with the widely accepted view that binaural responding depends upon the superior olive (SOC), with projections to the inferiof colliculus. Most neurons in the inferior colliculus show binaural sensitivity, including summation (EE/F) and suppreseion (EO/I) responses (Aitkin et al., 1975; Roth et al., 1978; Semple and Aitkin, 
1978), and the SOC is binaurally innervated, with LSO and MSO cells showing binaural responses similar to those in the inferior colliculus (Caird and Klinke, 1983; Guinan et al.. 1972a,b).

Neurons in the lateral superior olive (LSO) are excited by ipsilateral stimulation, eild inhibited by contralateral stimulation (IE cells) (Caird and Klinke, 1983). Thus contralateral excitation and ipsilateral inhibition of neural activity in the inferior colliculus could result from the major excitatory output of the lateral superior olive (LSO) to the contralateral inferior colliculus. Another potential source of binaural responses in the inferior colliculus comes from. the medral superior olive (MSO), which exhibits binaural sumation or suppression responses, and has a large ipsilateral projection (Inbody and Feng, 1981).

However, the binaural response patterns of the inferior colliculus (IC) can also occur independently from the superior olive. Recent experintents (Sally and Kelly, 1992) have shown that binaural sirniession (tro/i; and summation (EE) responses in the If presumed inportar.t in sound localization persist following destruction of the superior olivary complex with kainic acid, a neurotoxin which kills nerve cells tut spares fibers of passage (Masterton et al., 1979). In further studies (Li and Kelly, 1992), some neurons in the inferior solliculus remained sensitive to interaural intensity differences after unilateral or bilateral soc lesions. The remaining binaural inferior colliculus responses could be due to supraolivary binaural interactions.

Binaural interactions independent of the superior olive (SOC) could resuit from decussating trapezoid body fibers which bypass the SOC and 
project to midbrain structures that could mediate further binaural integration. For example, the DNLL receives fibers directly from the contralateral cochlear nucleus and sends fibers to ipsilateral and contralateral inferior colliculus. The inferior colllculus also receives a direct contralateral projection from the cochlear nucleus and sends crossed projections to the opposite inferior colliculus via comnissures (Adams, 1979; Beyerl, 1978; Brunso-Bechtold et al., 1981; Coleman and Clerici, 1987; Oliver, 1984, 1987; Shneiderman et al.. $1988)$.

Some experimental findings suggest that the $D_{1}$ 'LL may play a role in establishing contralateral dominance in neural responses of the auditory system. Most binaural neurons of the DNLL are excited preferentially by contralateral sounds, as are neurons in the inferior colliculus and auditory cortex (Aitkin et al., 1970; Brugge et al.. 1970). Most neurons in the DNLL are GABAergic and inhititory (Adans and Mugnaini, 1984; Thompson et al., 1985; Moore and Moore, 1987;

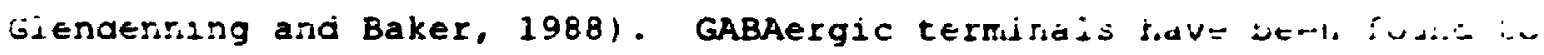
be interdigitated with potentially excitatory terminais fron oite: sources, along isofrequency laminae in the inferior colliculds. Thus: inhibition from the DNLL could enhance the contralateris dominance, or laterality, of binaural responses in midbrain or forebrain structures (Shrieiderman and Oliver, 1989; Oliver and Shneiderman, 1989: Shneiderman et al., 1988).

Further evidence shows that application of a GABA antagonist, bicuculline, can drastically affect binaural response characterietıcs of neurons in the central nucleus of the inferior coiliculus (IC). Thus binaural responses in the IC may have 1 rhibltory contributiens 
within the IC itself. Such contributions likely result from inhibitory projections from the DNLL (Faingold et al., 1989, 1991).

Recent evidence that the DNLL may be an important structure for binaural interactions in the inferior colliculus comes from experimerts in which kynurenic acid was used to suppress DNLI activity while binaural suppression responses in the inferior colliculus were monitored ( $L$ i and Kelly, 1991). These neurons wouid usually be inhibited by ipsilateral stimulation and excited by contralateral stimulation if they were given binaural input (EO/I). However, if activity in the DNLL contralateral to the inferior colliculus (IC) was suppressed, then ipsilateral stimulation would no longer produce inhibition in the IC to the same extent. The IC cells wers no longer as responsive to interaural intensity differences. Suppression of activity in the DNLi ipsilateral to the IC from which recordings were made did sot have this effect ( $\mathrm{Li}$ and Kelly, 1991).

\section{Pifpese of This study:}

Normative data on the binaural and monaural response characterastics of cells in the DNLL, VNLL and INLL of the rat were ottalned, using ilectrophysiological recording techniques. The greatest emphas : was placed on obtaining data from the DNLI, since this is a struct re which recent studies have implicated as important in sound localization. Attention was focused on obtaining a representative sample of cells of different binaural or monaliral types, and an attempt was made to observe any tonotopic organization, or segregation of different response types into different areas within a sucleus. Interaural intensity difference curves, frequency tuning 
clirves, and rate intensity curves were taken.

In contrast to the large amount of data accumulated on response characteristics of neurons in the cochlear nucleus, superior olivary complex, and inferior colliculus, the response characteristics of neurons in the lemniscal nuclei have been previously measured only in a few studies in the cat (Aitkin et al.. 1970; Brugge et al.. 1970; Guinan and Guinan, 1972a; 1972b) and bat (Covey and Casseday, 1991; Covey, 1993) and little is known about the functions of the lemniscal nuclei. Thus, such physiological data supplement knowledge on slie of the least known pathways in the auditory system, and allows a comparison of interspecies differences. 
Methods

\section{Anzmal Preparation:}

Electrophysiological data were obtained from 17 male Wistar albino rats (Rattus norvegicus) (250-350 g) ordered from Charles River Canada, St. Constant, Quebec. Surgical anesthesia was induced with sodium pentobarbital $(11.8 \mathrm{mg} / \mathrm{kg}$ i.p.), supplemented with sufficient Equithesin to induce a state of areflexia. Additional doses of Equithesin were administered approximately every 40 minutes to maintain areflexia (see Appendix B for preparation of Equithesin). Atropine sulphate $(0.05 \mathrm{mg} / \mathrm{kg})$ was administered prior to surgery to minimize respiratory distress.

Each animal was placed in a head holder which clamps the skull in place without use of ear bars so no damage to the tympanic membranes occurred. The skull was positioned so that lambda and bregma were at the same height. Following a midline incision and retraction of the scalp, a saniotomy was performed to expose the undrrlying cortex, and the did:a his retracted.

Three different procedures were used to locate, and insert the recording electrode into the DNLL, WNLL or INLL. In the procedure for the first set of experiments, a glass micropipette $12.0-2.5 \mu \mathrm{m}$ tip diameter) containing potamine sky blue dye $(3 \mathrm{~g} / \mathrm{mL}$ in Locke's solution) was inserted stereotaxically into position in either the left or right DNLL; :sing micromanipulators. The head of the animal was held flat, and the micropipettes were inserted at an angle of 30 degrees from vertical. The left and right DNLL were located using coordinates of the DNLI in three dimensions with reference to lambda IIi and Kelly, 1991). The micropipette was positioned with its tip at lambda, then 
raised $3.3 \mathrm{~mm}$ (in a direction 30 degrees from vertical), moved $0.4 \mathrm{~mm}$ more posterior, then moved $5.77 \mathrm{~mm}$ more lateral. At this point, the micropipette was $6.6 \mathrm{~mm}$ lateral to lambda and at the same vertical level as it. The micropipette was then penetrated $7.8 \mathrm{~mm}$, at a 30 degree angle from vertical, into the DNLL.

In the procedure for the second set of experiments, insulated tungsten $(2.0-2.5 \mathrm{M}$ impedance) electrodes were inserted in a vertical orientation, with the head of the arimal again held flat. The electrode was inserted at a point $0.4 \mathrm{~mm}$ rostral to lambda, and $2.75 \mathrm{~mm}$ lateral to lambda. The electrode was lowered $6.75 \mathrm{~mm}$ to the DNLL, and then inserted further ventral to pass through the INLL and the VNLL. Using this method, some electrode tracks passed through DNLL but not VNLl, or vice versa.

A third procedure was carried out to try to obtain recordings along a meciolateral direction. The electrode (insulated tungsten) was ruserted at an angle 30 degrees from vertical into the DNLL, to make recorjings from within DNLI, as well as structures located medial is. it, aiong the same electrode track. The coordinate system and methods Emfloyed to localize DNLL were the same as those described above for the fizst set of studies.

\section{Stimulus and Recording Technigues:}

Sine waves from a Hewlett Packard function generator (HP 3310 A) staped by a Coulbourn Instruments gate (584-04) formed tone burst: of $110 \mathrm{msec}$ duration including $10 \mathrm{n} / \mathrm{sec}$ rise and decay times. The stimulus repetition rate was $1 / \mathrm{sec}$. The shaped signal was split and passed through two independent passive decade attenuators (Hewlett Packard 
35001.

The signals were amplified using an Aiwa (P30) power amplifier, and transduced by Pioneer SE-5OD stereo headphone drivers mounted in customized housings. Each earphone housing opened into a small acoustic coupler that was connected directly to the rat's external auditory meatus. The sound pressure levels in each ear were measured with a probe tube that was inserted into the couplers to a point within a few millimeters of the tympanum. Measurements of the acoustical crosstalk between the two ears with the drivers still coupled to the rat's external meatus had revealed a value of $65 \mathrm{~dB}$ SPI attenuation up to $1.3 \mathrm{kHz}$, and at least $70 \mathrm{~dB}$ for higher frequencies, in experiments using the same sound delivery system (II and Kelly, 1991 ).

The rat was situated inside an electrically shielded, sound attenuated room. The electrical signal from each microelectrode was amplified 10,000\% using a Bak Electronics pre-amplifier and amplifier (MUA-3i, and monitored using an audio amplifier and a storage cs. $+1+1$ cscove.

fition potential spikes from single neurons were selected using a time amplitude window discriminator (Bak Electronics DIS-1), and an arajog deiay linit (Bak Electronics AD-6). Acceptance pulses were stored using a Tracor Northern signal averager (TN-1550), to produce post-stimulus time histograms, and the action potential waveforms were monitored on a storage oscilloscope.

\section{Experimental Procedures:}

Normative physiological data on NLL neurons were obtained. For each seuron encountered, the characteristic frequency (CF) was 
determined for sound at the contralateral ear, with no sound presented to the ipsilateral ear. The characteristic frequency was operationally defined as that frequency for which the lowest sound pressure level of contralateral stimulation would elicit spikes. The CF was found by determining the range of frequencies at which stimulus-synchronized spike responses occurred, while sweeping the function generator acrcss the cell's excitable frequency range at progressively lower contralateral sound pressure levels, until a response was obtained for only one frequency (characteristic frequency). If the neuron was excited by sound at the ipsilateral ear, then the CF was determined for sound only at the ipsilateral ear. If the neuron was excited by stimulation in either ear, then the characteristic frequency was determined independently for sound at either ear.

Classification of binaural response properties of each neuron was determined by delivering the CF tone (assessed contralatezally) at different sound pressure levels to each ear indeperdently, and $x ;$

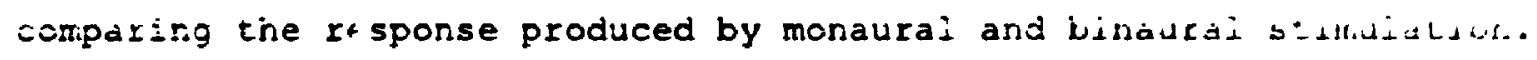
The contralateral sound pressure level was held constant ar:d tone ipsilateral sound pressure increased in 5-10 dB steps to determirc whether ipsilateral stimulation inhibited response to contralaterel sound. If some neurons were found which were inhibited or excited ty sound at jow sound pressure levels, then this was also an indication. that the ear drivers were properly placed in the two ears.

The response sound pressure threshold level, the sound level at which the response occurs approximately 50 of the time, was determired differently depending on the binaural response classification of ite neuron. For monaural (EO/O or OE/O) cells, the threshold was 
determined for excitation with sound at one ear (the contralateral ear for EO/O, ipsilateral ior OE/O). FOr suppression (EO/I) cells, the contralateral threshold was determined with sound only to the contralateral ear; the ipsilateral threshold for inhibition was found with sound at the contralateral ear set at 10-20 dB SPL above the contralateral threshold. For summation (EE/O or EE/F) cells, ipsilateral and contralateral thresholds was measured by delivering the CF tone to each ear independently, and varying sound pressure levels to determine the level at which the response occurred 508 of the time.

Tuning curves were obtained for neurons with contralaterally driven responses (EO/O, EE or EO/I) by measuring the sound pressure threshold level at different frequencies within the cell's responsive area.

Rate intensity curves, showing the number of action potential spikes as a function of sound pressure level of stimulus ir. one ear, were also accumulated for a sample of neurons. To facilitate comparison among units with very different firing rates, the data for each neuron were normalized to its own maximum evoked spike rate. A judgement was made as to whether rate intensity curves were monotonic or non-monotonic. A monotonic function can be operationally defined as one in which an increase in sound level results in an increased or stable firing rate; a non-monotonic function is one in which an increase in sound level at some point results in a decrease ir firing rate of at least 108 (Covey and Casseday, 1991).

Sensitivity to interaural intensity differences (IIDs) in EO/I (OF EI) cells was determined by measuring the response of single neurons to systematic manipulation of the relative sound pressure in the two ears. Contralateral sound pressure level was fixed at 10-20 dB above 
threshold and ipsilateral level was incremented in 5 or $10 \mathrm{~dB}$ steps over the cell's dynamic range. Counts of spikes which occurred during the 110 msec tone burst over 50 stimulus presentations, were accumulated in post-stimulus time histograms, at each relative sound pressure in the two ears. An IID function (percentage of maximum spike count as a function of interaural intensity difference) was calculated for each neuron.

Neuronal responses were also characterized by response latency (the amount of time for the first response in the post-stimulus time histograml, and the pattern of response spikes occurring during each stimulus presentation (identified from the fost-stimulus time histogram). For example, a single spike or a few spikes occurring at stimulus onset with little or no activity afterwards constitutes an onset response, while a train of spikes constitutes a sustained response. A train of spikes, followed by a pause, followed by a second train of spikes, is termed an onset-pause-sustained response IIrvine, ISgE!

Cnset, onset-pause-sustained, or sustained responses may show choppirg; choppers have regularity in intervals between spikes, which is usually evidenced by modes in the post-stimulus histogram. In order to assess whether or not a neuron shows a chopping response, the mean interspike interval, which allows one to study changes in the intervals between spikes during the time course of the stimulus, can be computed fe.g., Bourk, 1976; Smith and Rhode, 1989; Smith and Rhode, 1987; Smith et al., 1951; Rhode, Smith and Oertel, 1983; Rhode, Oertel and Smitr, 1983). In this measure, all spikes in response to tone bursts are piaced in 200 microsecond bins based on their time after tone onset. 
The time to the next spike for each $s_{i j} i k e$ in a given 200 microsecond bin is measured and the mean interval for the spikes in that bin is calculated (Smith and Rhode, 1989). Intervals for bins toward the end of the response, where the spikes are often not followed by another spike until the onssi of the next tone, are not plotted. When plotted versus time after stimulus onset, the mean interspike interval for sustained choppers usually is fairly constavt or gradually increasing for the duration of the stimulus. Rarely, the interval between the first two driven spikes will be noticeably shorter than later ones (Smith and Rhode, 1989). Onset choppers with some sustained response often show an abrupt increase in mean interval corresponding to the transition from well-timed onset peaks to the sustained portion of the post-stimilus histogram, which shows less chopping; this is sometimes called a "chop-T" response (Covey, 1993) (e.g.. onset chopper with sustained, Figure 81 . For these chop-T responses, the regularity of firing is decreasing with time after stimulus onset. The coefficiert cf vaniztion fequal to the standard deviation in the mean interspito interval, divided by the mean interspike intervall is another index of chopping; values below 0.5 are considered to indicate chopping l Covey. $1993 i$.

While lack of a computerized system for on-going analysis of interspike intervals precluded the calculation of these quantitative measures of chopping, post-stimulus histograms accumulated for different contralateral sound pressure levels and frequencies of tone burst stimulation permitted classification of some neurons as choppers in the present study. 
Dye Injection or Current Lesion and Histology:

To mark the locations at which recordings were made from neurons, for those experiments in which glass micropipettes were used, the dye in the glass pipette was injected iontophoretically by applying a current of $\theta$ microamperes for 20 minutes. In experiments in which insulated tungsten electrodes were used, a small lesion was made at the top and bottom of the electrode track, by applying a curzent of 8 microamperes for 75 seconds. The animal was perfused through the heart with saline followed by 10 formalin. The brain was removed, and frontal sections, taken at 40 micron intervals and stained with cresyl violet, were used to verify the recording sites. For the experiments where lesions were used, the position of each neuron along the electrode track was inferred from the settings of the micromanipulator that was used to drive the electrode during the experiment. The distance between the two lesions on the brain sluces was expected to be:

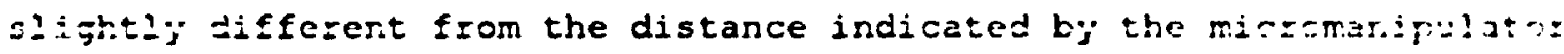
settings, because of tissue shrinkage during histological procedires. Therefore, a scaling factor was used to calculate the position of each neuron between the two lesion sites. 


\section{Results}

Recordings were made from a total of 198 neurons, localized using either iontophoretic dye injection or electrolytic lesions in the DNLL or surrounding lemniscal structures. Nineteen electrode penetrations (cases BU17, BU18, BU20, BU27-R, BU27-L, BU30, BU38, BU41, BU43, BU45, BU46-L, BU46-R, BU50, BU52, BU55, BU56, BU59, BU60, BU61) were made in a total of 17 animals.

Six cases in whith iontophoretic dye injections were used to localize the recording sites (BU17, BU18, BU20, BU27-R, BU27-I, BU30) included 35 single unit recordings from the DNLL. For each of these cases, the neurons which were recorded were only considered to have been localized to the DNLL if the dye injection site was located in the central region of the DNLI. These cases did not yield any information as to the spatial distribution of response types within the DNLL, and single units from these cases would be expected to be located Fredcrirastys in the central regions of DNLL.

In cases where vertical electrode tracks were used and electrolytic lesscns were used to localize neurons, recordings were made from a total of 114 neurons, sampled from seven electrode tracks (cases Bu38, BU43, SU46-I, BU46-R, BU50, BU41, and BU45) in six animals (Figure 4 ). cf these recordings, all were single unit recoraings, except for two multi-unit records of neurons in ventral DNLL in case BU50 (CF=21.4 $k H z, C F=24.5 \mathrm{kHz}$, and three multi-unit recordings from sagulum in case BU46-R (CF=8.00 kHz, $C F=3.76 \mathrm{kHz}, C F=5.82 \mathrm{kHz})$. In all cases in which multz-unit recordings were made, only two neurons were concurrently recorded, and the responses of the two units could easily be 
Figure 4A-M. Frontal sections showing reconstructed vertical and oblique electrode tracks (cases BU3B, BU41, BU43, BU45, BU46-R, BU46-I BU50, BU52, BU55, BU56, BU59, BU60 and BU61), which may proceed through the inferior colliculus (and perhaps through the central nucleus of the inferior colliculus, ICC), then pass more ventrally through the DNLL, sagulum (SAG). INLL or VNLL. Characterietic frequencies (kHz) and binaural response type (EO/I, EO/O or EE) of neurons recorded elong each track are shown. Distance of each track along the rostral-caudal axis (measured from the level at which the combsure of Probst has the largest number of fibers entering and connecting with the DNLL; this level of interaural axis is used as the zero referencel is shown in microns. Negative values designate caudal tracks, while positive values designate more rostral tracks. Cases are presented in a sequence from caudal to rostral. 
A. BU38 $-240 \mu \mathrm{m}$

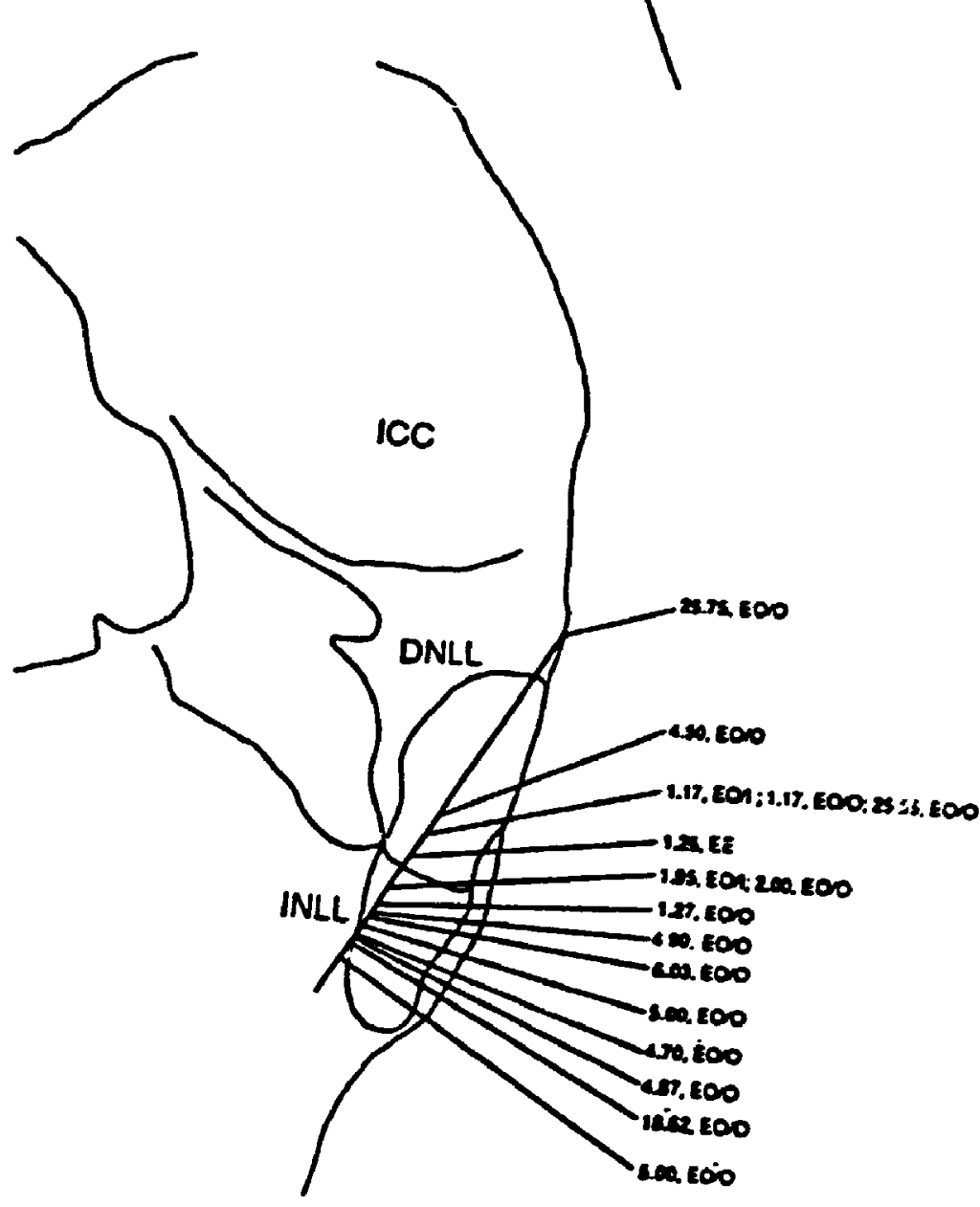

B. BU56

$-160 \mu \mathrm{m}$ CONTINUED OVER 
C. BU46-R $-120 \mu \mathrm{m}$

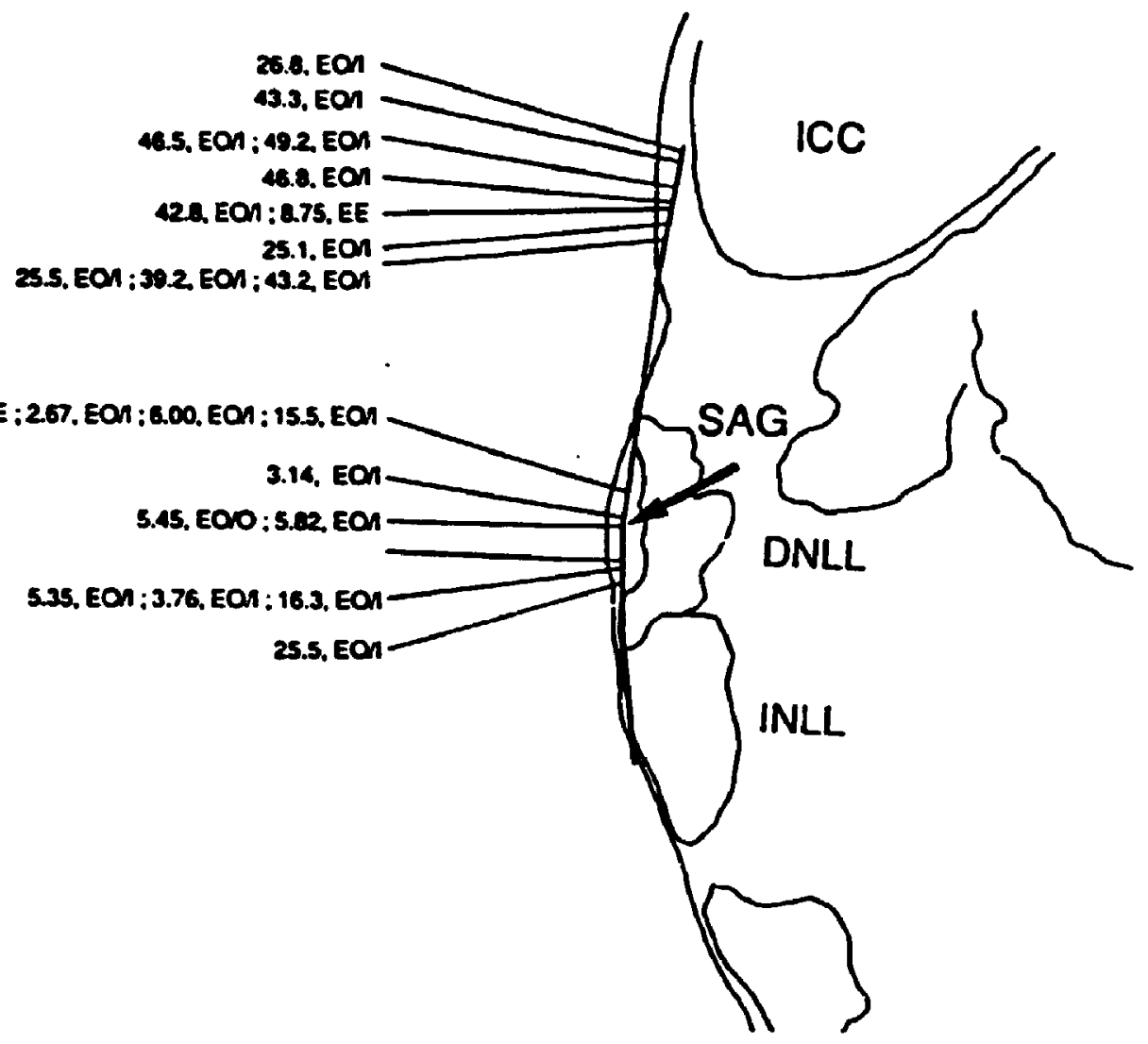

D. BU41

$-80 \mu \mathrm{m}$

8.00, EE ; 2.67. EON ; 6.00, EON : 15.5. EON 5.45, EOOO : 5.82 EO

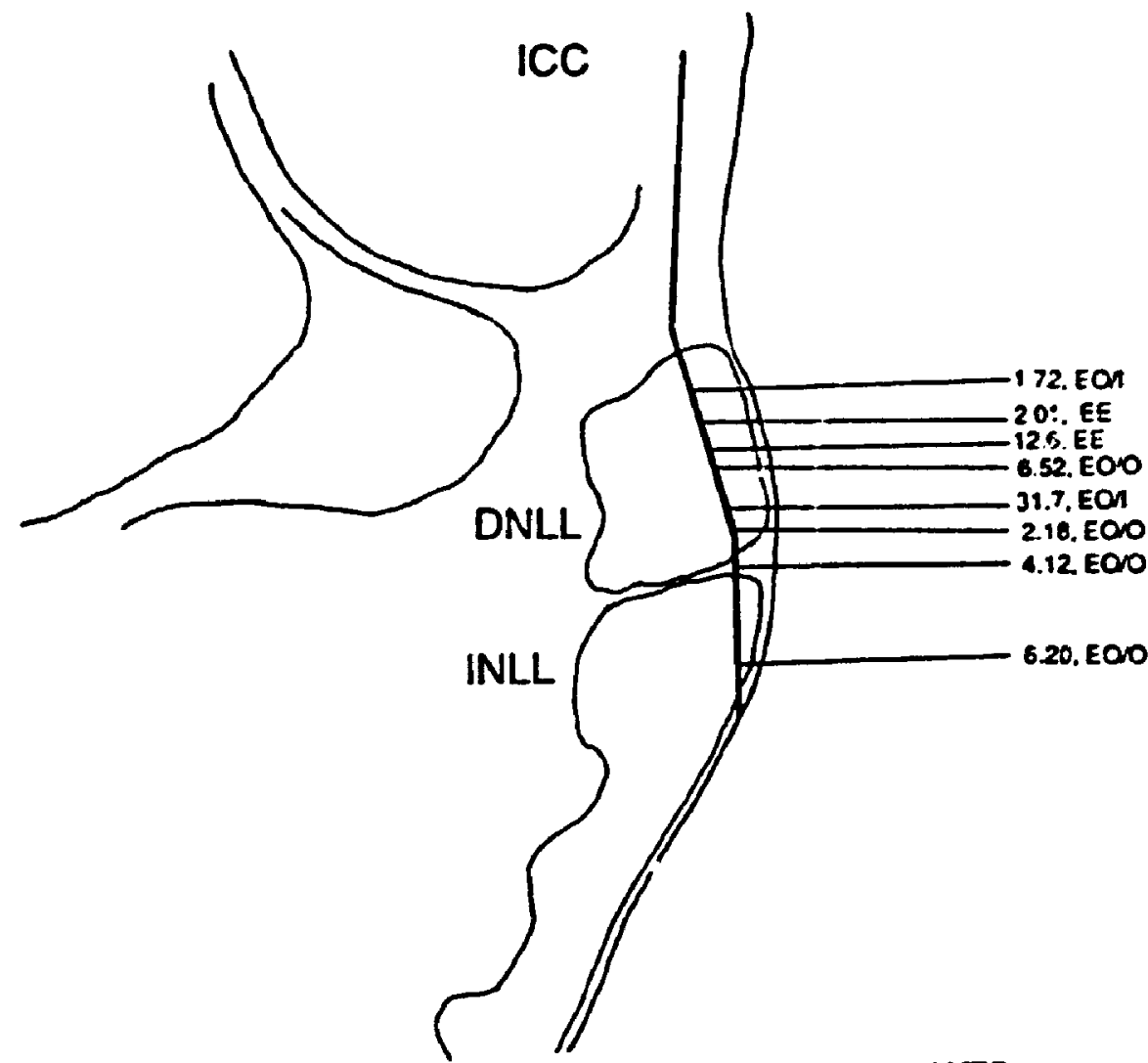

Pigure 4. (continued) 
of EC/I Or EE neurons were located at the medial, dorsal and ventral margins of INLI (Figure $5 b$ ).

Paralemiscal zone:

The regions of reurons medial to INLL and DNLL showed predominantly EO/I resporses. However, electrode track BU46-L, which was a track at the media: ma $n$ of DNui, and has not been included in this map since it is quite far rostral, would show exclusively monaural Eo/o responses in a medial region. Since the paralemiscal zone extends quite a bit more rostrally than DNLL, the more rostral track (BU46-L) passed through the more central part of this region.

Rostral-Caudal Distribution of Binaural Sensitivity: DNLI

While dorsal-vertral or medial-lateral progressions in binaural tjpes of neurcns were not very clear, a segregation of binaural atspo:sf types along the rostral-caudal axis of DNLL was evident.

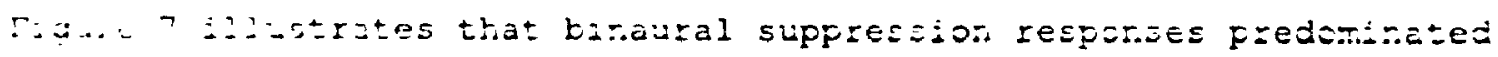
In a central region of ENLL, while further rostraliy or caudally, monaural responses were present in higher proportions. The certral inatural reaicn of LNLL (in which EO/I responses predomaratedi seems to coistspoid to the rostral-aadal extent thrcugh which the commssure of Frobs: projects into the DNLL (Flgure 7). These commissural fibers are Fojecticris from the DNiz on t!e opposite side, and thus this result coild imply that neurons having EO/I suppression responses project to ithe optosite orist.

Cadial thestrode tracks ltaken at levels 60 microns to 240 microns sudai to the zelo cefezerce level at which the comissure of probst 


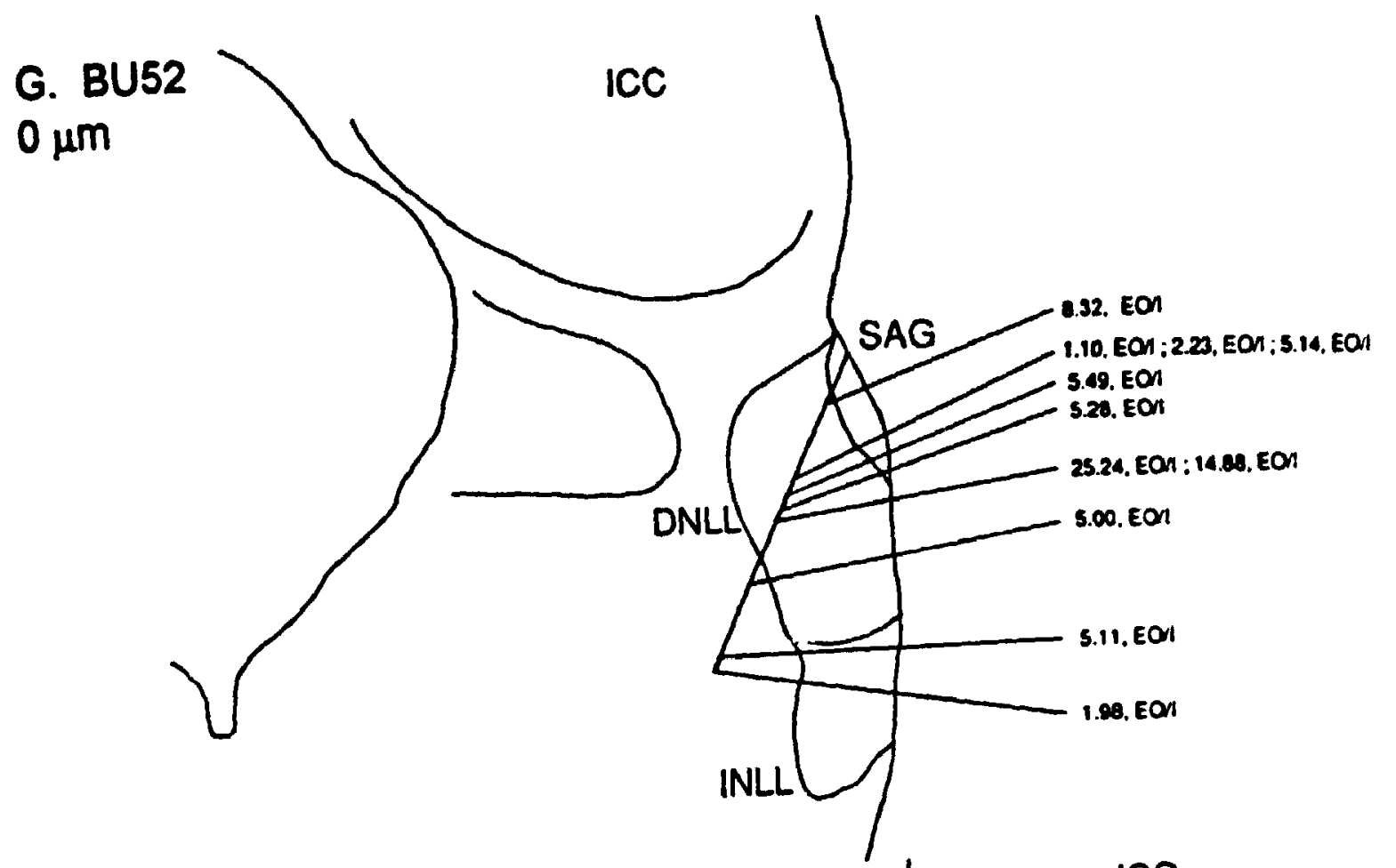

H. BU45

$+120 \mu \mathrm{m}$

133, EOn : 0.54, EOn ; 4.18, EOn ; 11.43, EE : 128, MUXED 1.11. MIXED
1.26. EON
1.26. EON : 6.59. EE 0.70 , EE : 12.1. EE ;5.55, EE 20.5. EOn : 16.8. EOn ; 13.0. EOn ; 8.98, EOH

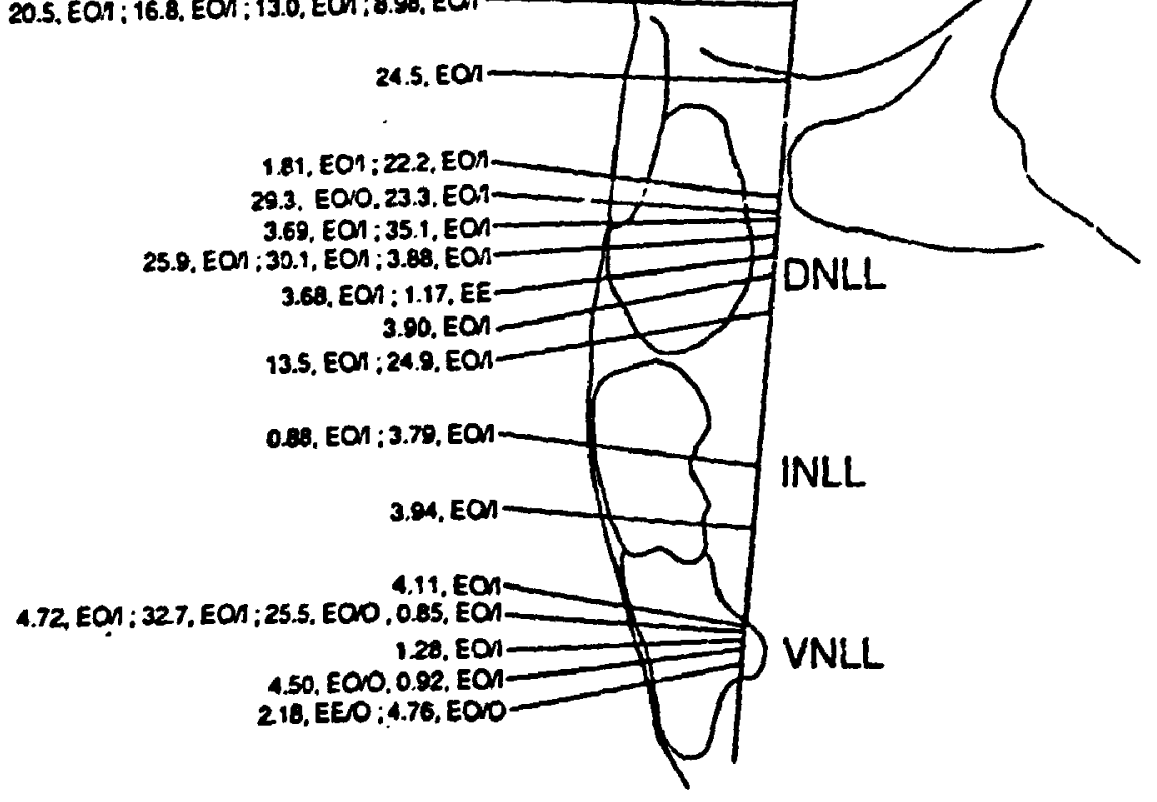

Eigure 4. (continued) 


\section{BU59}

$+160 \mu \mathrm{m}$

J. Bบ4்3

$+140 \mu \mathrm{m}$

$1000 \mu \mathrm{m}$
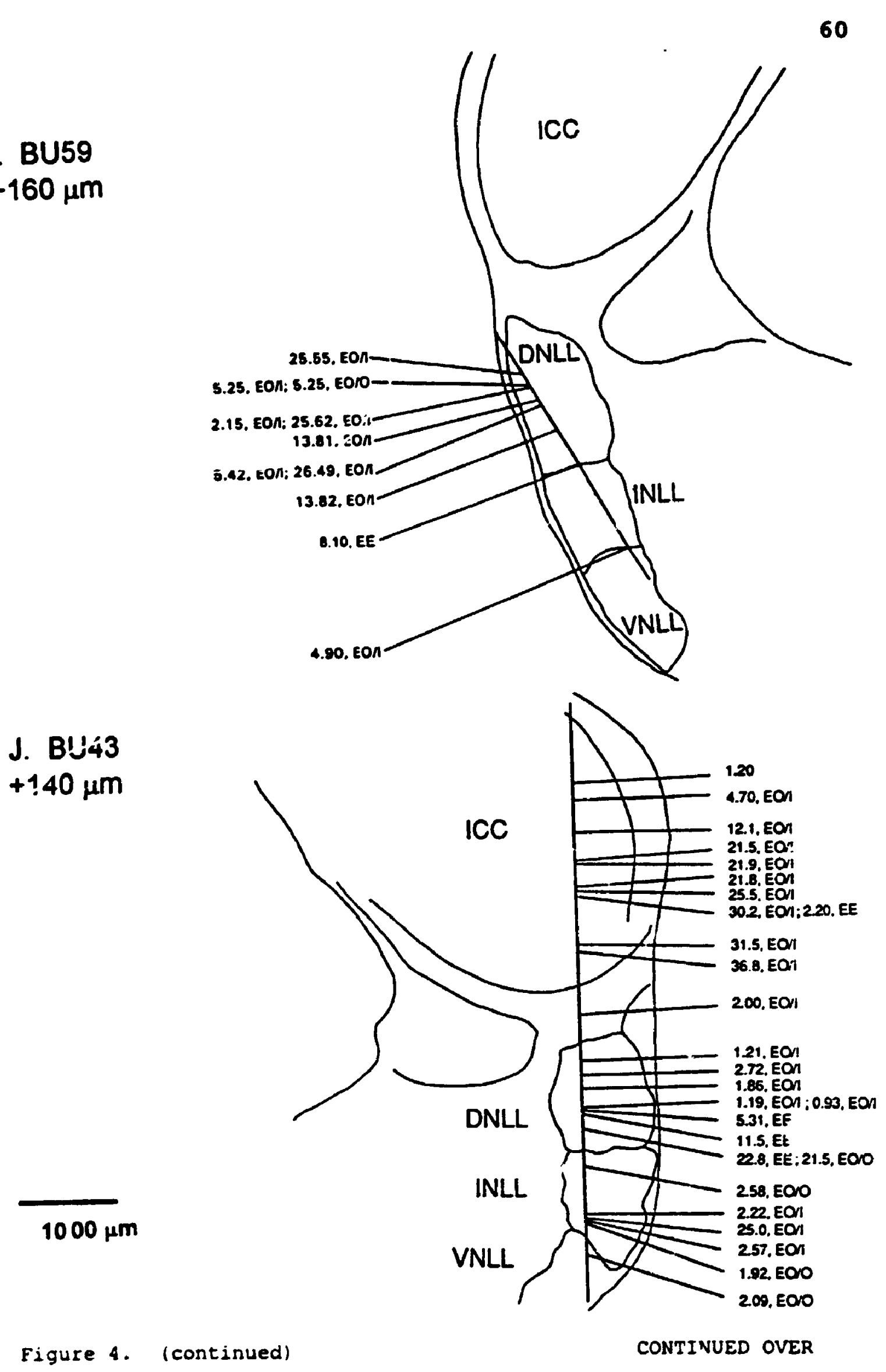


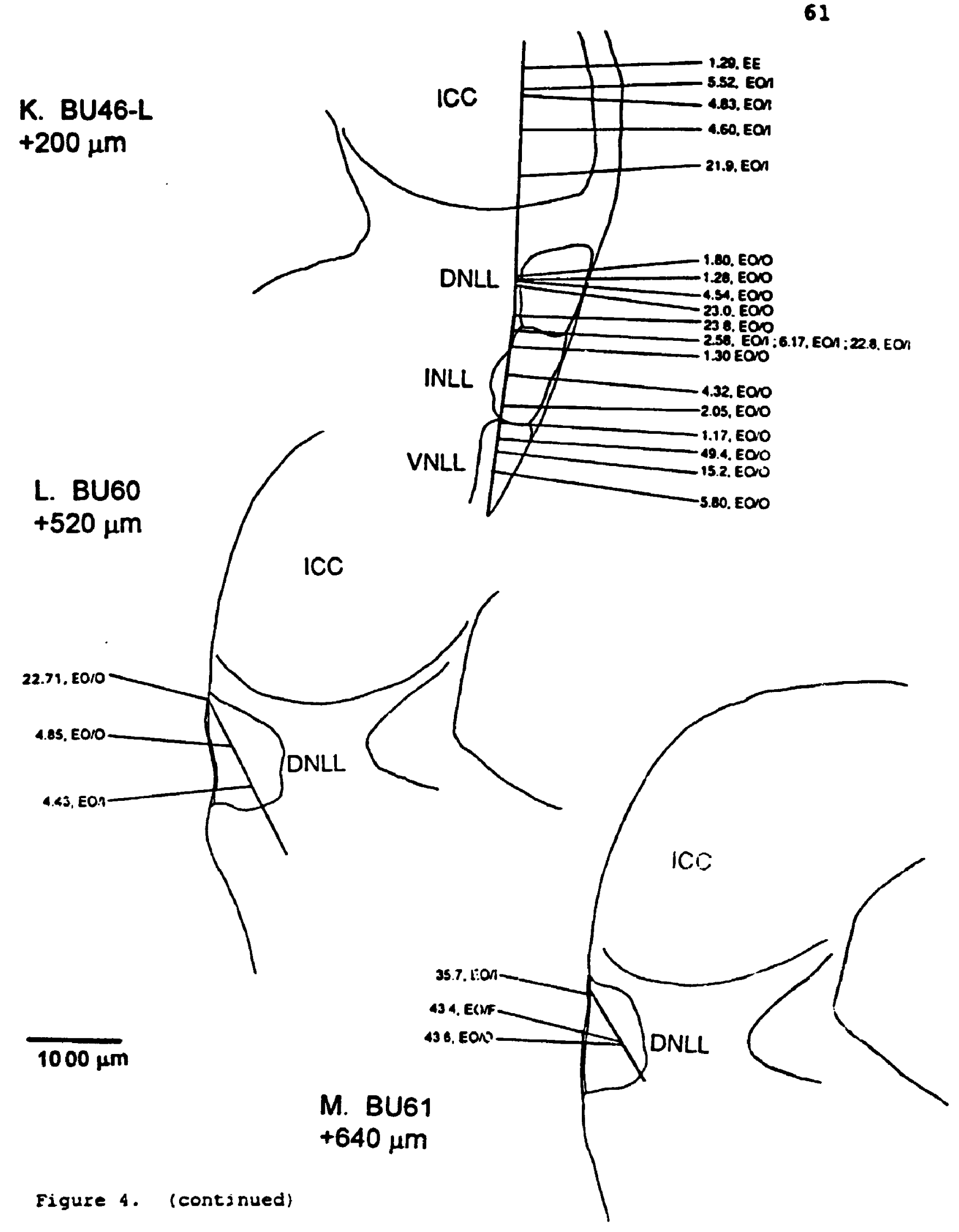


distinguished and separated using the window discriminator.

In the remaining six cases (BU52, BU55, BU56, BU59, BU60, BU61), 49

single units were recorded using tungsten electrodes along oblique

electrode tracks (at a 30 degree angle from vertical), and neurons were localized using electrolytic lesions (Figure 4).

\section{Tonotopic Organization:}

DNLL:

In contrast to the reliable characteristic frequency progression within the inferior colliculus, no linear frequency progression was evident within DNLL. This becomes evident from individual electrode tracks, shown in Figure 4. Frequency progressions in the inferior colliculus showed increasing characteristic frequencies from dorsal to ventral (Figure 4, BU45, BU43, BU46-L, BU50). While a few individual electrode tracks showed a dorsal to ventral progression of increasing characteristac frequency within DNLL (Figure 4, BU50, BU43), some t:acr.s showed high and low characteristic frequencies close together (e.g. Figure 4, BU59, BU41), or had no apparent frequency progression. A composite diagram in which data from all electrode tracks lexcluding far rostral of caudal tracks) were projected onto a single reprosentative section, collapsing across the rostral-caudal axis, also shows no obvious frequency progression (Figure 5 ).

\section{Binaural Response Properties:}

DNLL:

The 100 nedrons localized in the DNII in a? "electrode penetrations, but excluding extreme rostral electrode tracks (BU60, 
Table 1A-B. A. The numbers of units which were recorded in different regions in the lemniscal pathway which showed different binaural response types $(E O / I, E O / O$ or EE) are shown. B. The numbers of units recorded from different lemniscal structures which had different temporal response patterns (Onset, response at onset; Sust, sustained; On/Sus, Onset followed by a pause and sustanned spike train) are shown. Numbers of neurons are shown for DNLL, rostral DNLL lincludes only extreme rostral tracks BU60, BU61), regions dorsal to DNLL, regions medial to DNLL, INLL, regions medial to INLI, VNLI, and for sagulum (SAG).

A.

FREQUENCY OF BDAURAL RESPONSE TYPES DN LEMNISCAL PATHWAY

\begin{tabular}{|c|c|c|c|c|c|c|c|c|}
\hline & DivL & $\begin{array}{l}\text { Romer } \\
\text { DNLL }\end{array}$ & $\begin{array}{l}\text { Doned is } \\
\text { DNLL }\end{array}$ & $\begin{array}{l}\text { Niniwlo } \\
\text { DNLL }\end{array}$ & NLL & $\begin{array}{l}\text { Modw co } \\
\text { WNLL }\end{array}$ & VNLL & SAC \\
\hline EON & 74 & 2 & 3 & 17 & 12 & 5 & 7 & 9 \\
\hline $20 / 0$ & 17 & 3 & 0 & 1 & 22 & 0 & 8 & 1 \\
\hline $\mathbf{E E}$ & 9 & 0 & 1 & 1 & 1 & 0 & 1 & 1 \\
\hline EO/F & 0 & 1 & 1 & 0 & 0 & 0 & 0 & 0 \\
\hline عسח EOR & 0 & 0 & 1 & 0 & 0 & 0 & 0 & 0 \\
\hline
\end{tabular}

B.

FREQUENCY OF TEMPORAL RESPONSE TYPES DN LEMNISCAL PATEWAY

\begin{tabular}{|c|c|c|c|c|c|c|c|c|}
\hline & DNL & $\begin{array}{l}\text { Podred } \\
\text { DNLL }\end{array}$ & $\begin{array}{l}\text { Dornd a } \\
\text { DNLL }\end{array}$ & $\begin{array}{l}\text { Motwe 1 } \\
\text { DNLL }\end{array}$ & NLL & $\begin{array}{l}\text { Mealw wo } \\
\text { DLL }\end{array}$ & VNLL & SAG \\
\hline Ooset & 52 & 1 & 3 & 17 & 2 & 2 & 2 & 11 \\
\hline DrSust & 20 & 1 & 3 & 2 & 4 & 2 & 5 & \\
\hline inst & 19 & 4 & 0 & 0 & 27 & 1 & 9 & \\
\hline
\end{tabular}


BU61), showed a majority of binaural suppression responses 174.08 EO/I), with smaller proportions of EO/O monaural responses $(17.0 \varepsilon)$, or EE summation responses $(9.05)$ (Figure $6 a$, Table 1$).$

The two rostral DNLL tracks (cases BU60 and BU61) have been treated separately from the central DNLL in the analysis of binaural properties, since they showed lower proportion of EO/I neurons compared with EO/O neurons, and also included one neuron with a facilitatory binaural response type (EO/F) not seen elsewhere in DNLL in this study (EO/I cells, 33.38 of a sample of 6 neurons; EO/O cells, 50.08; EO/F cells, 16.74). The EO/F neuron found in rostral DNLL did not respond to ipsilateral sound stimulation: however, cortralateral stimulation produced excilation, an excitation which increased if ipsilateral sound was also present. Rostral DNLL thus showed binaural sensitivity different from that fcund in central DNLI; however, the small sample size ( 6 neurons) of rostrally localized neurons may limit the con:iusions that can be drawn.

Requors Da:sal to the Mann Subdivision of DNLL:

The regien dorsal to the main subdivision of DNLL but ventral to the inferior colliculus, an area with few cells, was found to show binalra: sensitivity (Figure 4, cases BUt5; BU50; BU43). The largest proportion of neurons showed EO/I suppression responses $(40 \%$ of a sample of S), although other binaural response types were present 120 \& EO'F: 20* EO/I mixed; $206 \mathrm{EE}$ ). 
Figure 5A-C. A. Composite reconstruction to illustirate the locations of single and multi-units with given characteristic frequencies, shown in units of $\mathrm{kHz}$, in the nuclei of the lateral lemniscus. Dita from all experimental animals lexcept for extreme rostral and caudal tracks BU60, BU61. BU46-I and BU38) were pooled and projected to a single representative frontal section. B. Composite reconstruction done as in (A), but illustrating the distribution of binaural response characteristics of neurons in the lemniscal nuclei. C. Composite reconstruction as in (A), but illustrating the distribution of temporal response patterns, and only including single units for which poststimulus histograms accumulated at different frequencies of stimulation allowed definite characterization. Neurons are shown as non-choppers if any uncertainty existed as to whether the neuron exhibited chopping.

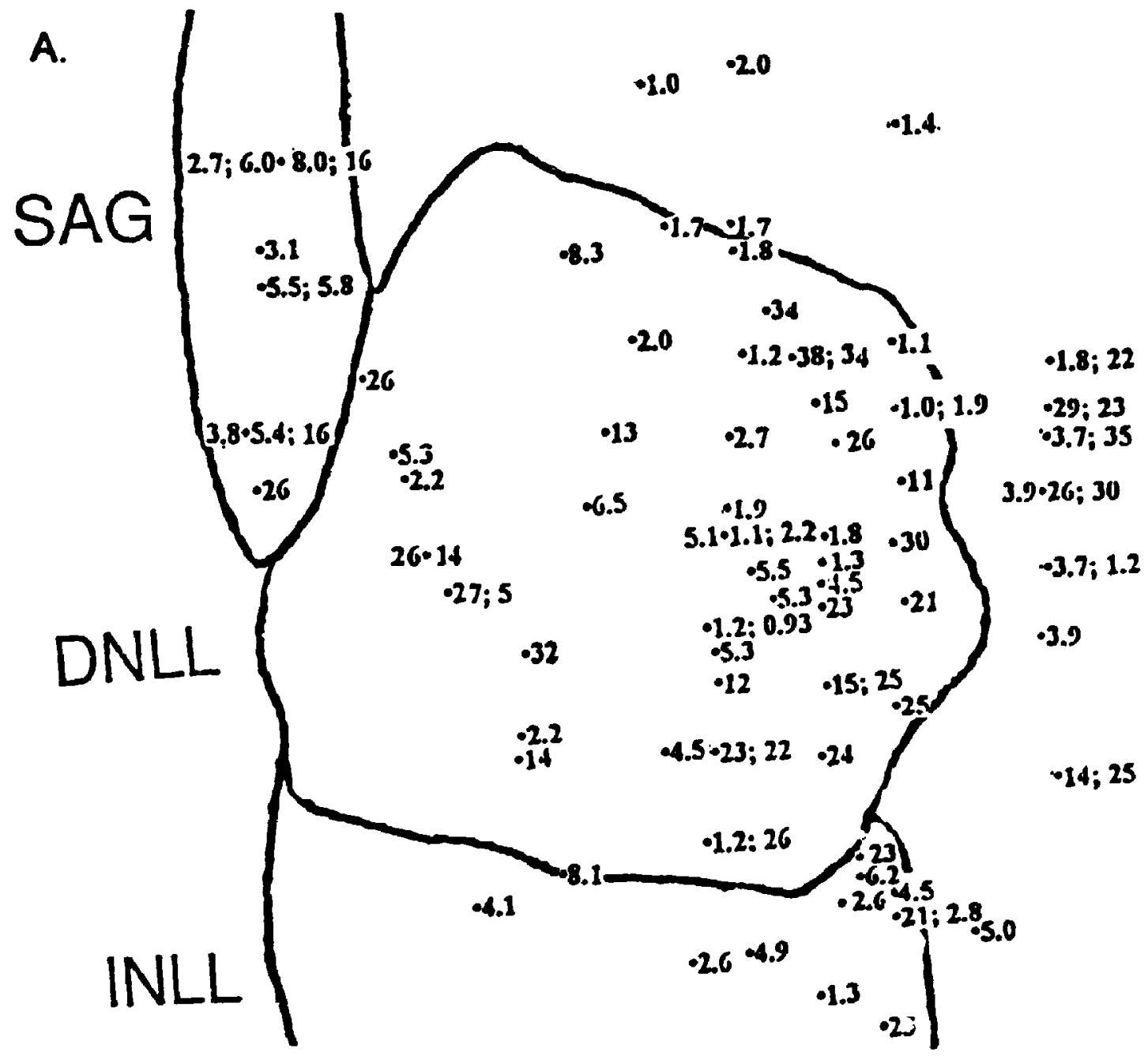


Figure 5A-C. A. Composite reconstruction to illustrate the locations of single and multi-units with given characteristic frequencies, shown in units of $\mathrm{kHz}$, in the nuclei of the lateral lemiscus. Data from all experimental animals lexcept for extreme rostral and caudal tracks BU60, BJ61, BU46-L and BU38I were pooled and projected to a single representative frontal section. B. Composite reconstruction done as in (A), but 11lustrating the distribution of binaural response characteristics of neurons in the lemniscal nuclei. C. Composite reconstruction as in (A), but illustrating the distribution of temporal response patterns, and only including single units for which poststimulus histograms accumulated at different frequencies of stimulation allowed a definite characterization. Neurons are shown as non-choppers if any uncertainty existed as to whether the neuron exhibited chopping.

B.

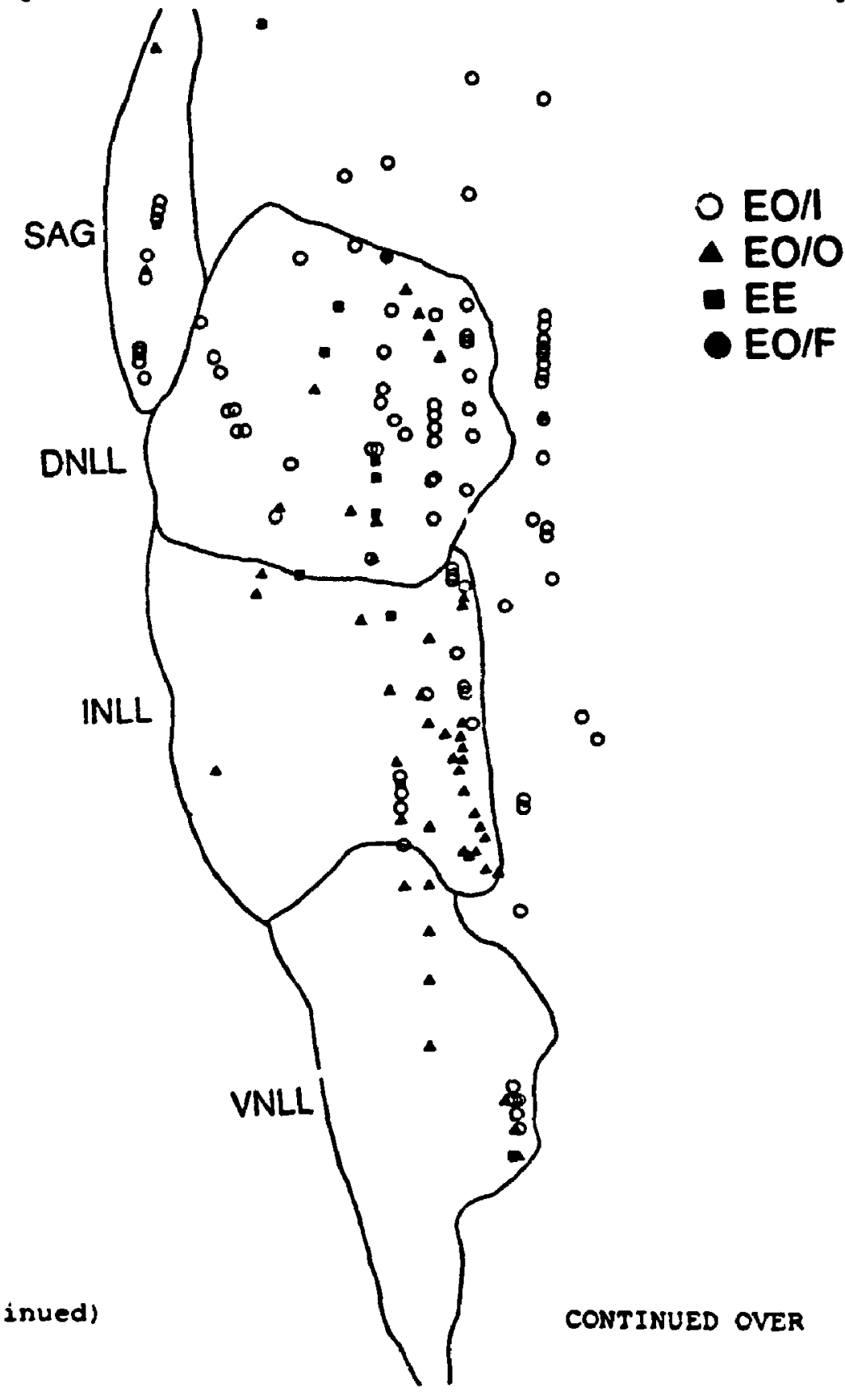

Figure 5. (continued) 
Fiqure A-C. A. Composite reconstruction to illustrate the locations of $5^{\circ}$.gle and multi-units with given characteristi: frequencies, shown in units of $\mathrm{kHz}$, in the nuclei of the lateral lemiscus. Data from all experimental animals (except for extreme rostzal and caudal tracks BU60, BU61, BU46-I and BU38) were pooled and projected to a single representative frontal section.

B. Composite reconstruction done as in (A), but lllustrating the distribution of binaural response characteristics of neurons in the lemiscal nuclei. C. Composite reconstruction as in (A), but illustrating the distribution of temporal response patterns, and only including single units for which post-stimulis histograms accumulated at different frequencies of stimulation allowed a definite characterization. Neurons are shown as non-choppers if any uncertainty existed as to whether the neuron exhibited chopping.

C.

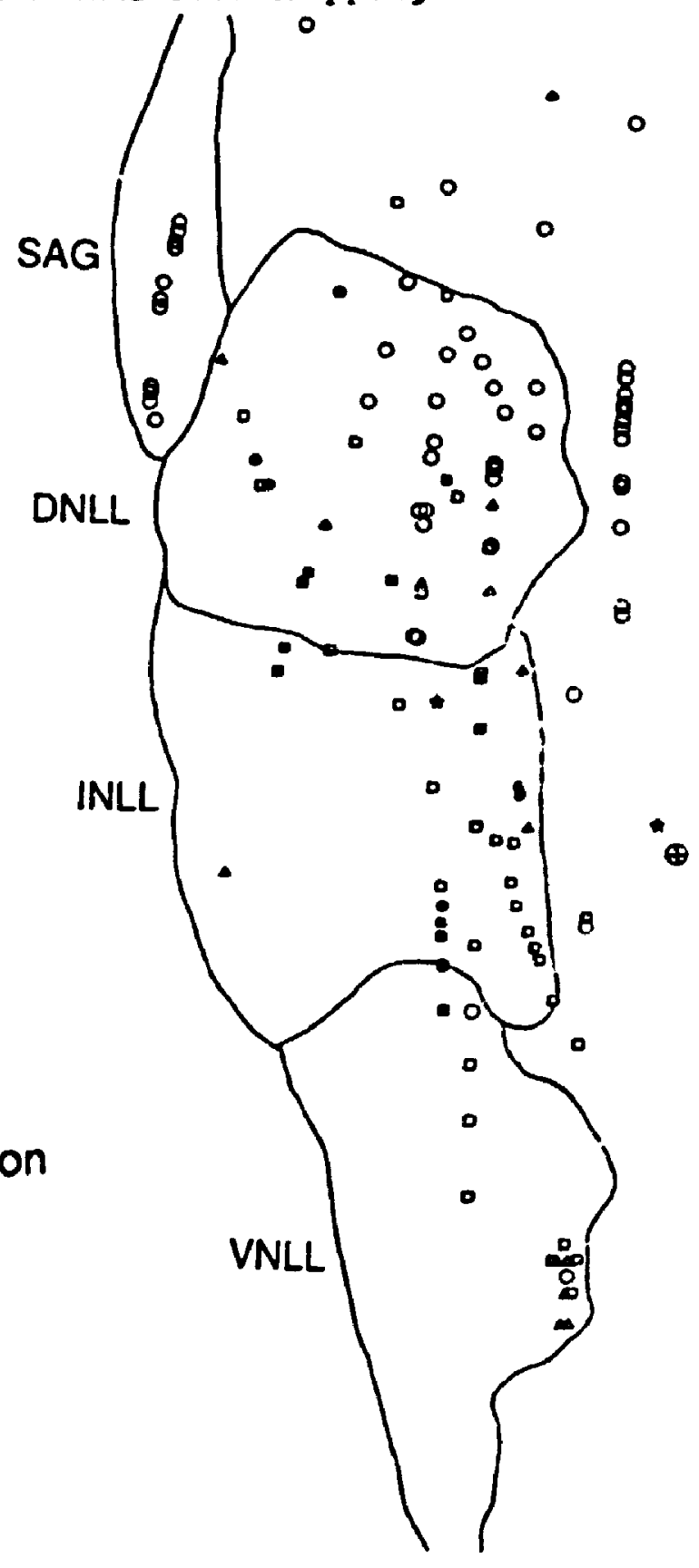

C Onset

- Onset chopper

$\triangle$ On-pause-sust

- On-pause-sust chopper

- Sust

Sust chopper

$\oplus$ Other: sust after offset

* Other: onset with habituation

Eigure 5. (continued) 
Figure 6A-B. A. Percentages of binaural response types (EO/I, EO/O, EE) In DNLL, INLL and sagulum, obtained with vertical and oblique - lectrode tracks (cases BU38, BU41, BU43, BU45, BU46-R, BU46-L, BUSO, BU52, BU55, BU56, BU59), but excluding the two extreme rostral tracks, BU60 and BU61. B. Percentages of temporal response types obtained with vertical and oblique electrode tracks (same electrode tracks as in A.) (Onset, response at onset; Sust, sustained; On/siss, onset followed by a pase and a sustained spike train) obtained $i: i$ DNLL, INII and sagulum. Only neurons definitively localized within conventional cytoarchitectonic boundaries of DNLI, INLL and sagulum were included.

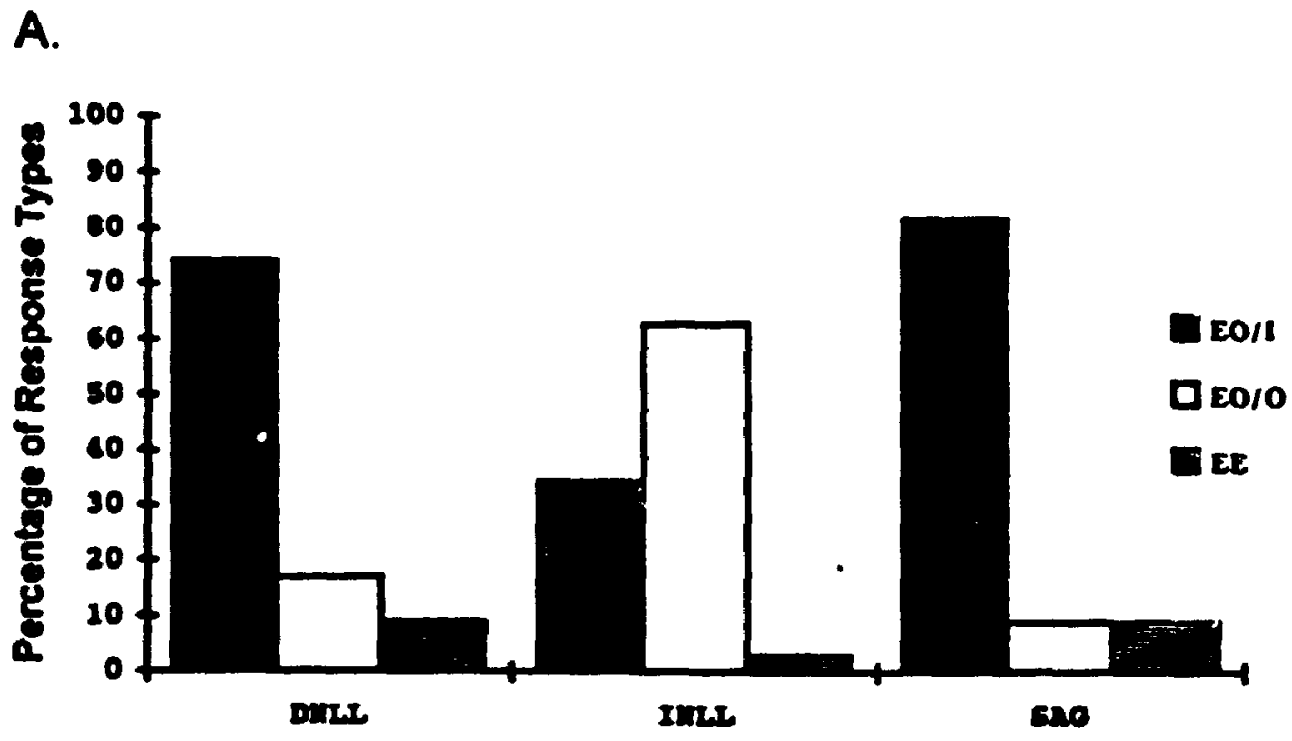

B.

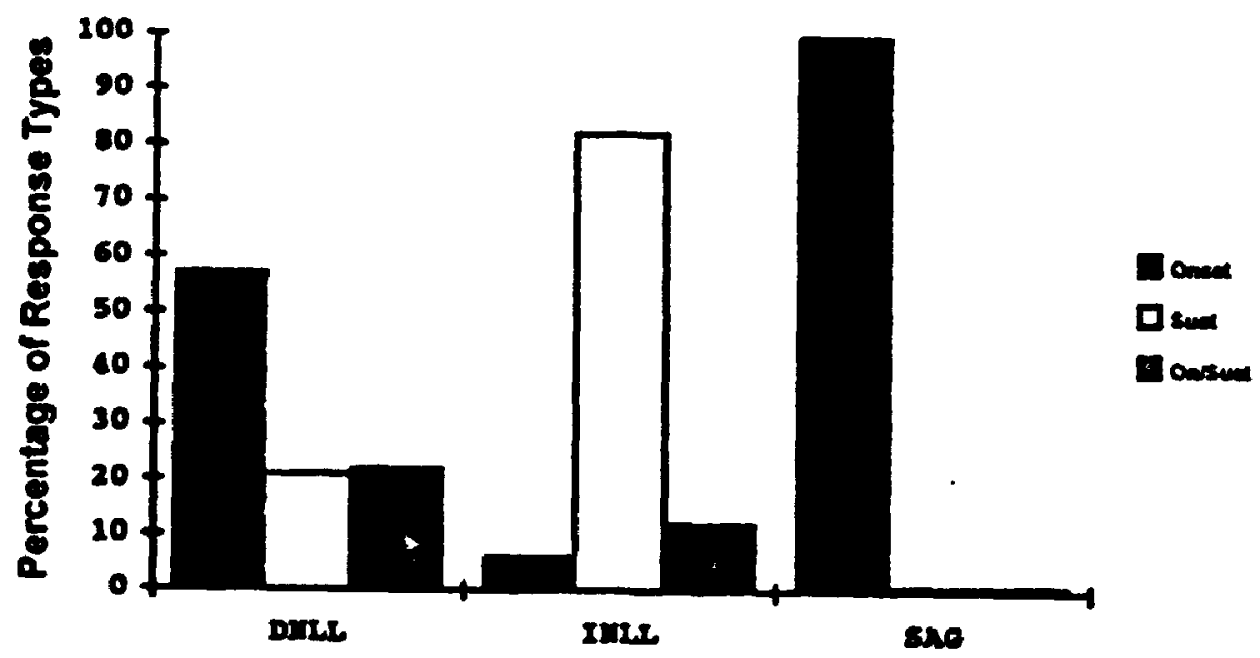


Saguium:

Binaural sensitivity simslar to that in DNLL was found in sagulum neurons in case BU46-R; a majority of EO/I suppression responses was found $(81.88 \mathrm{EO} / \mathrm{I}$ in a sample of 11$)$.

INLL:

In contrast to the predominance of binaural suppression responses in DNLL and sagulum, the majority of INLL neurons were monaural EO/O neurons (62.9f of a sample of 35), while a significant fraction were binatral EO/I (34.3: of 35) and 1 neuron in dorsal INLI was a binaural sumration EE cell in case BU59 (2.86) of 35) (Table 1. Figure fial).

\section{VNLL:}

The sample of VNLL neurons was not large enough or well enough distributed over the whoie nucleus to obtain proportions of binaural respoise iypes. The data from VNLL inciuded exclusiveiy morausai : 0 'O: $^{\prime}$ resporses obrained from central or dorsal reglons of vill lcase Eu43, I netrcn; case BU46-1, 4 neurons) but also included reurons from a medial regica which stower binaural sensitivity (mostly EO/1 responses) (case BU45, 10 neurons). In case BU59, a sirgle EO/I neuror. was localized to VNII, and this neuron was located dorsomedially (Table 1).

\section{Paralenriscal Zone:}

Regions medial to DriL and IliLL showed bunaural sersitiolty and a predominance of suppression responses, with 89.5. EO/I cells 117 of a 
E2gure 7. Percentage of different binaural response typiss (ro/I, EO/O, EE) found along the rostrocaudal axis through DNLL. The ceritral part of the comissural connections to DNLL lor zero point on $x$-axis in the Figurel was taken as the point at which the density of fibers entering in the DNLL was the greatest. However, the comissural filier connections with DNLL extend further rostrally than caudally from this point. The extension in the rostral-caudal dimension over which the commssure of Probst fibers enter the DNLI is shown by the bar undernearh the figure.

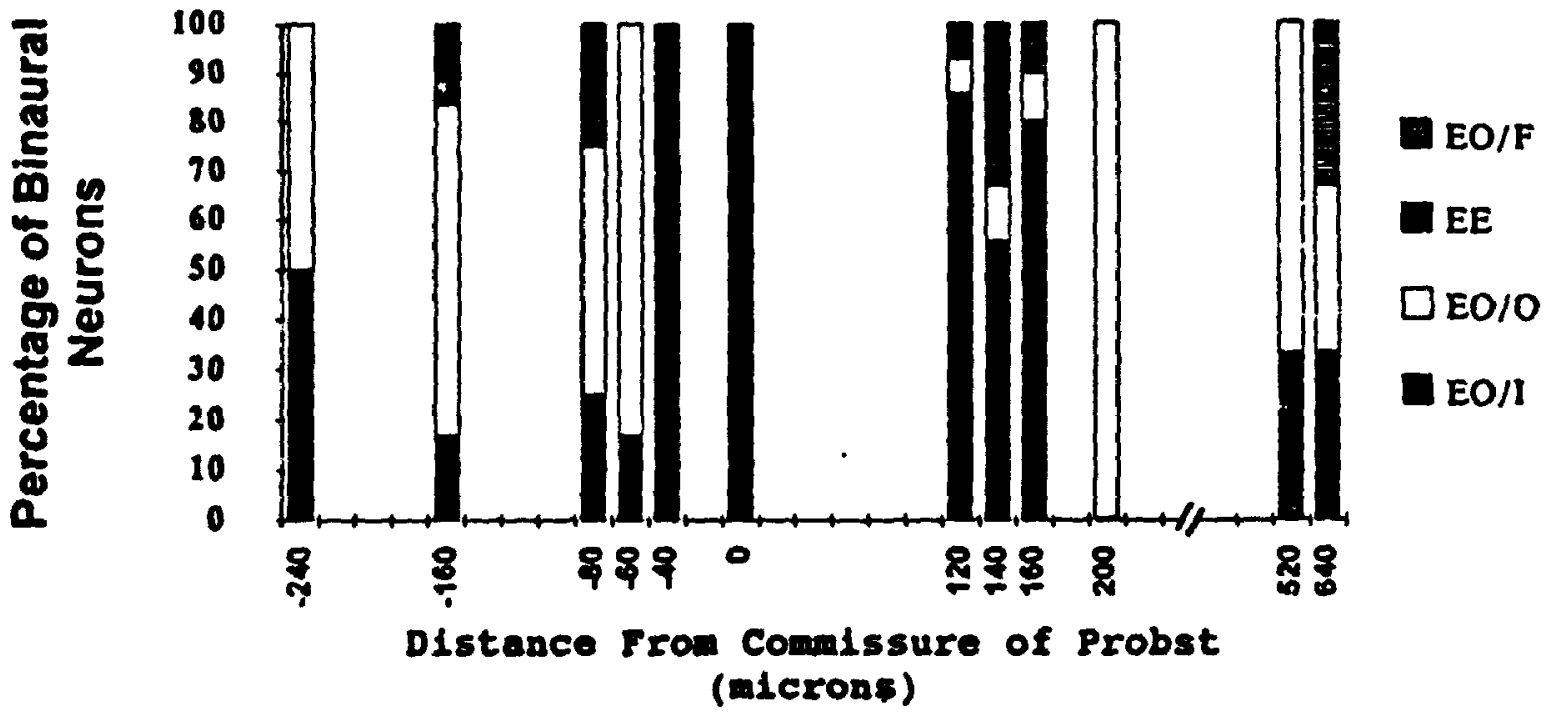

Exteat of Commissure of Probst at DNLL 
has the densest connections with DNLL) showed predominanti' monaural EO/O responses (range of 50 to $16.7 \%$ EO/I responses). Electrode tracks passing through the central division of DNLL (from 40 microns caudal to the interaural axis, to 160 rostral to this point) showed a predominance of EO/I suppression responses 1100 t of a sample of $9 ; 100$, of a sample of $7 ; 85.7 \varepsilon$ of a sample of $14 ; 80 \%, N=10 ;$ and $55.6 \%, N=9$ ). The three rostral electrode tracks showed a predominance of EO/O monaural responses, although sample sizes were smaller $1100 \%$ Eo/O with $\mathrm{N}=6 ; 66.7 \mathrm{EO} / 0$ with $\mathrm{N}=3 ; 66.78 \mathrm{EO} / \mathrm{O}$ and $\mathrm{EO} / \mathrm{E}$, with $\mathrm{N}=3$ ).

In summary, binaural suppression EO/I responses were found in the central part of DNLL (when considering the rostral-caudal dimension), while higher proportions of EO/O monaural responses were found elther rostrally or caudally. The central region in which electrode tracks showed highest proportions of EO'I neurons extended over approximately 200 microns, and the caudal and rostral borders of this region matched with the caudal and rostral extent of commissural projections to ihe arpate mit.

Rostral-Caudal Distribution of Binaural Sensitivity: Faralemriscai Zorit The rostrai-caudal distribution of hinaural sensitivity $2 r_{i}$ the region medial to DNLI aid INLL was found to parallel buraural sensitivity in the DNLL i.tself. The rostral track (BU45-L) included criy monaural resporses clcse to the medial margin of UNLL, while mor*. caudal tracks (BU45, BU52) located at the same rostral-caudal level te the central binaurally responsive region of LliLi, sncwed exclusively EO/I suppzession responses. There therefore siems to haye beth a rostral-caudal segregation of biratial response types in the region. 
of EO/I or EE neurons were located at the medial, dorsal and ventral margins of INLL (Figure Sb).

\section{Faralemniscal zone:}

The regions of reurons medial to INLI and DNLL showed predominantly EO/I resporses. However, electrode track BU46-I, which was a track at the medial ma $n$ of $\mathrm{DNL}$, and has not been included in this map since it is quite far rostral, would show exclusively monaural Eo/O responses in a medial region. Since the paralemniscal zone extends quite a bit more rostrally than DNLL, the more rostral track (BU46-I) passed through the nore central part of this region.

Rostral-Caudal Distribution of Binaural Sensitivity: DNLI

While dorsal-ventral or medial-lateral progressions in binaural types oi neurons were not very clear, a segregation of binaural respo:sst types along the rostral-caudal axis of DNLL was evident.

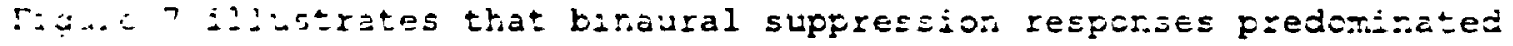
Ir. a central region of ENLL, while further restrally or caudally, monaural responses were present in higher proportions. The certral bunaural reaion of DNLL (in which EO/I responses predominated) seems to colzespoid to the rostral-caidal extent through which the commissure of Frobst projects into the DNLL (Figure 7). These commissural fibers are projections from the DNLL on the opposite side, and thus this result co:id $2 \mathrm{mply}$ that neurons having EO/I suppression responses project to the opposite DNiI.

Caucial electrode tracks ltaken at leveis 60 microns to 240 microns caudal tc the zero reference level at whach the comissure of Frobst 
Figure $\theta$. Post-stimulus histograms illustrating different temporal response patterns from single units in DNLL or INLL. A. Onset inonchopper, spikes at onset of tone burst). B. Onset chopper $(2-3$ spikes at onset of stimulus). C. Onset-pause-sustained response, no evidence of chopping (spontaneous activity present). D. Onset-pause-sustained response with chopping (spontaneous activity present). E. Sustained response, with chopping in the onset component of the response. Also could be viewed as an onset chopper with sustained component. F. Sustained response, no evidence of chopping (spontaneous activity present). G. Sustained, slow-chopping response. The duration of the stimulus tone burst was $110 \mathrm{msec}$ in every case.

A.

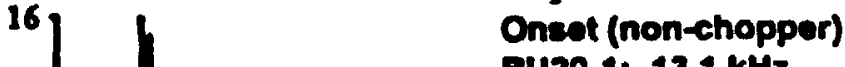

B.

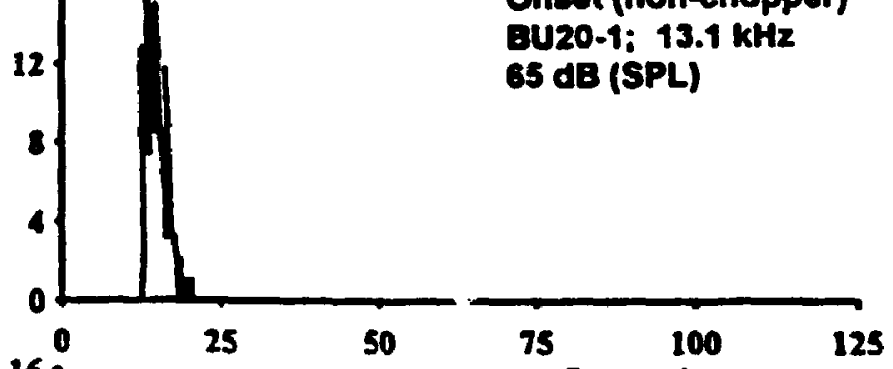

B.

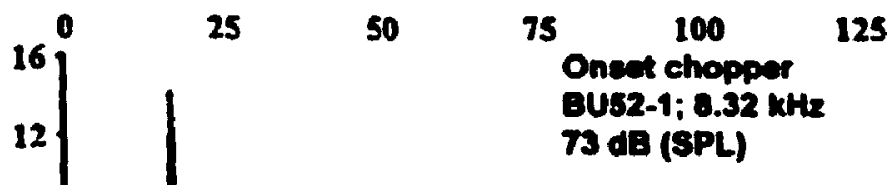

C.
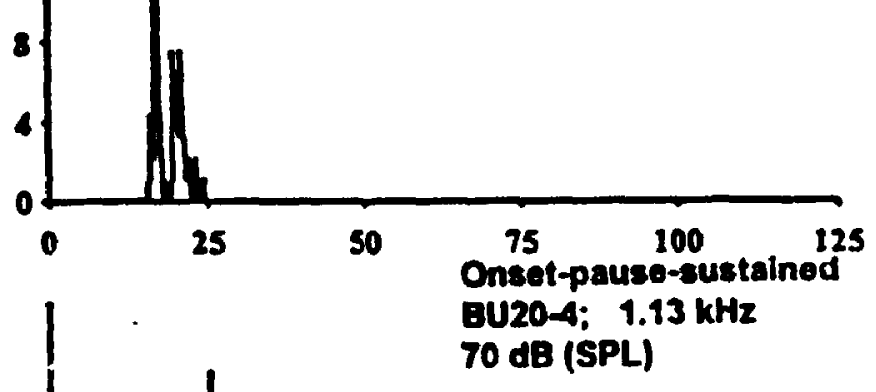

D.

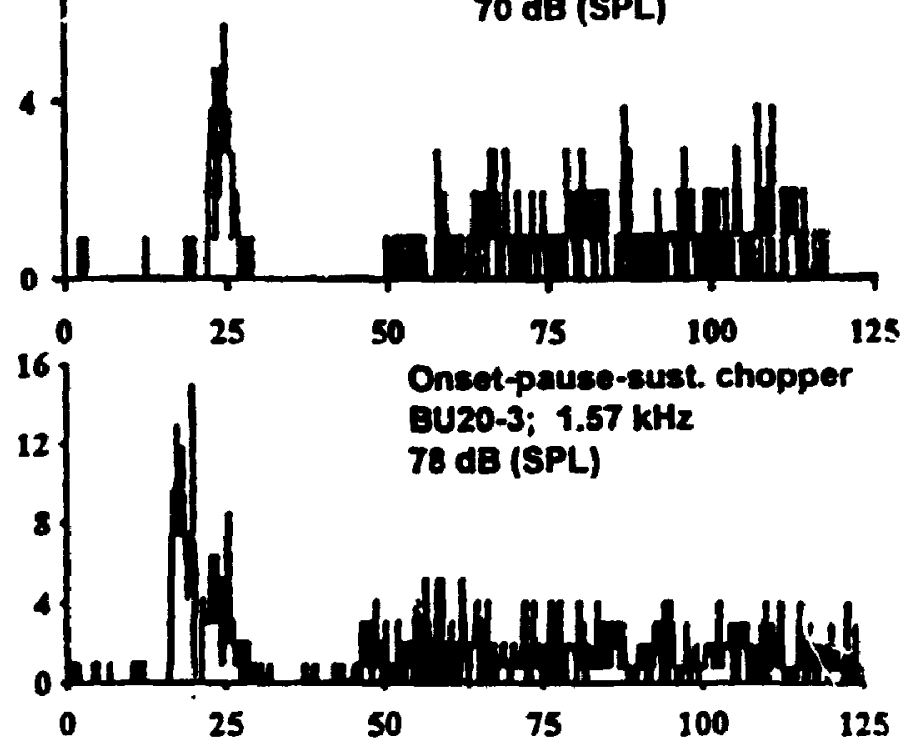


E.

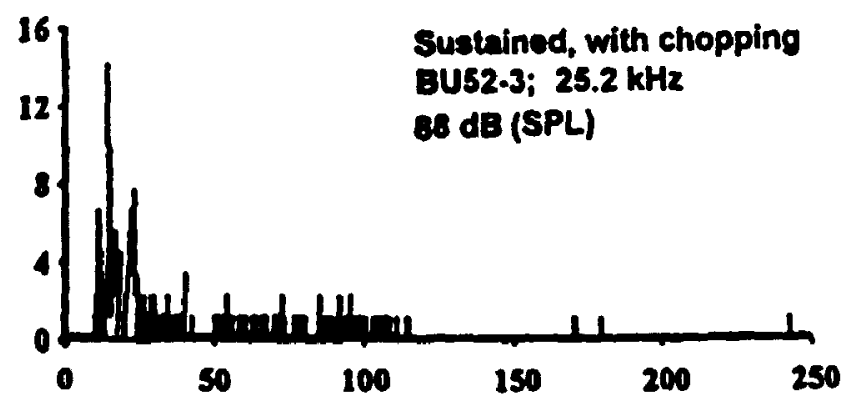

F.

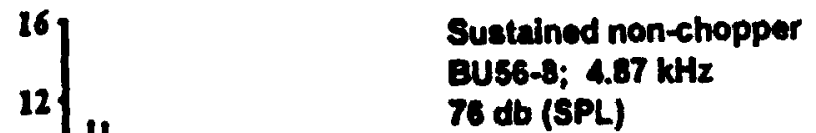

G.

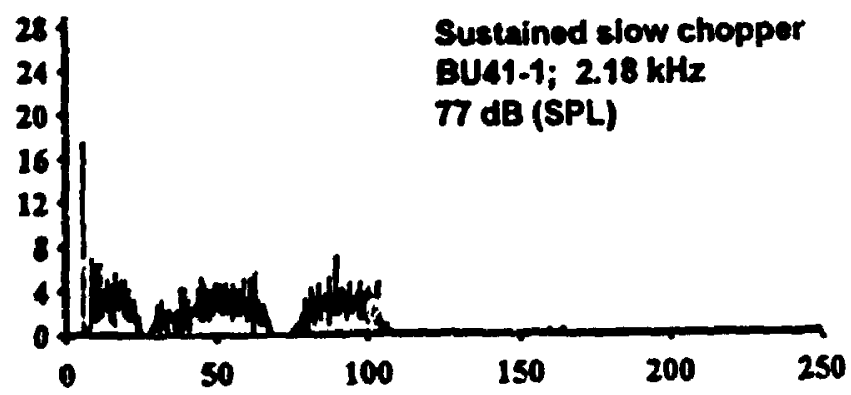

Figure 8. (continued) 
has the densest connections with DNLL) showed predominantiy monaural EO/O responses (range of 508 to $16.78 \mathrm{EO} / \mathrm{I}$ responses). Electrode tracks passing through the central division of DNLL (from 40 microns caudal to the interaural axis, to 160 rostral to this point) showed a predominance of EO/I suppression responses (100t of a sample of 9; 1004 of a sample of $7 ; 85.7 \varepsilon$ of a sample of $14 ; 80 \%, N=10$; and $55.6 \%, N=9)$. The three rostral electrode tracks showed a predominance of EO/O monaural responses, although sample sizes were smaller $1100 \%$ EO/O with $N=6 ; 66.78$ EO/O with $N=3 ; 66.78$ EO/O and EO/E, with $N=3$ ).

In summary, binaural suppression EO/I responses were found in the central part of DNLL (when considering the rostral-caudal dimension), while higher proportions of EO/O monaural responses were found elther rostrally or caudally. The central region in which electrode tracks showed highest proportions of EO'I neurons extended over approximately 200 microns, and the caudal and rostral borders of this region matched with the caudal and rostral extent of commissural projections to the arpacits Dnit.

Rostral-Caudal Distribution of Binaural Sensitivity: Faralemniscal Zone

The rostrai-caudal distribution of hinaural sensitivity in the region medial to DNLI aid INLL was found to parallel binaural sensitivity in the DNLI itself. The rostral track (BU46-L) included cniy monaural resporses clcse to the medial margin of DNLL, while more caudal tracks (BU45, BU52) located at the same rostral-caudal level to the central binaurally responsive region of LllLI, sncwed exclusively EO/I suppzession responses. There therefore stems to have been a rostral-caudal segregation of binaural response types in the region 
medial to DNLL as well as in DNLL.

\section{Temporal Response Patterns:}

DNLL:

Figure $6 b$ illustrates that of the 91 units in DNLL llocalized with dye injections, or from reconstructed vertical or oblique electrode tracks) for which temporal response patterns could be verified, a majority (57.18) showed onset responses, and significant proportions had onset-pause-sustained responses $(22.09)$ or sustained responses $(20.94)$.

Typical post-stimulus histograms for different temporal resporse patterns found in DNLL or INLL are shown in Figure 8 . While a certain proportion of either onset, onset-pause-sustained, or sustained responses in DNLL showed chopping, whether or not a neuron was a chopper neuron could not always be ascertained, since the experimentai apparitus did not allow computer frocessing of intervals between spikes as the: w-re accumulated in post-stimulus histograms.

Whether or not a neuron showed chopping could sometimes be ascertained if post-stimulus histograms had been accumulated at different irequencies of stimulation, and for differr. $t$ sound pressuze levels; for a sample of 5 onset neurons in central DNLL which responded with several spikes at onset, the majority $(808)$ were found to show onset chopping. A significant proportion of onset respnnses only responded with 1 spike at onset 110 neurons mainly located in dorsal or medial DNLL), and therefore could not have shown any chopping, but the possiblity that for other stimulation conditions, the neuron would have shown a chopping response, can never be totally eliminated. For a 
Fiqure 9A-B. A. Distribution of latencies (msec) to first spike response, in response to pure tone bursts at $C F, 10 \mathrm{~dB}$ above threshold, for 49 single unit neurons located in DNLL, including all rostralcaudal levels. B. Distribution of latencies (msec) to first spike response (measured as in (A)) for different binaural response types (EO/I, EO/O, EE, EO/F) found in DNLI at all rostral-caudal levels.

A.

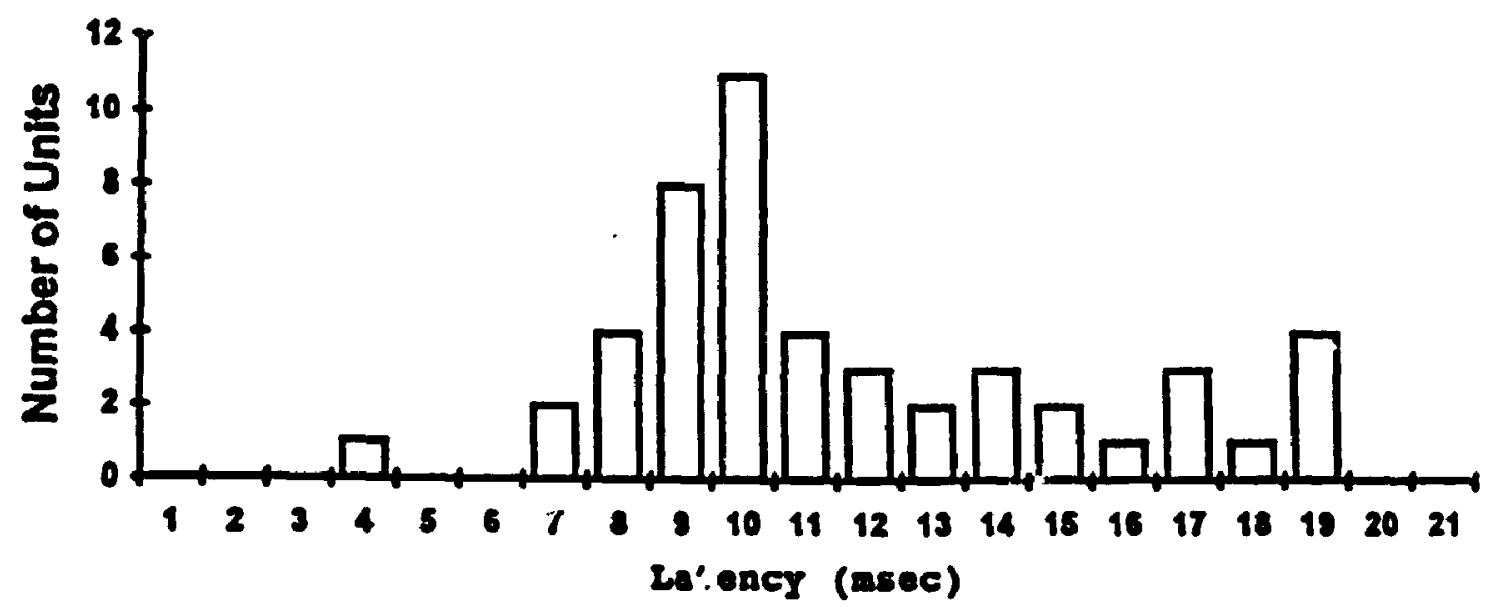

B.

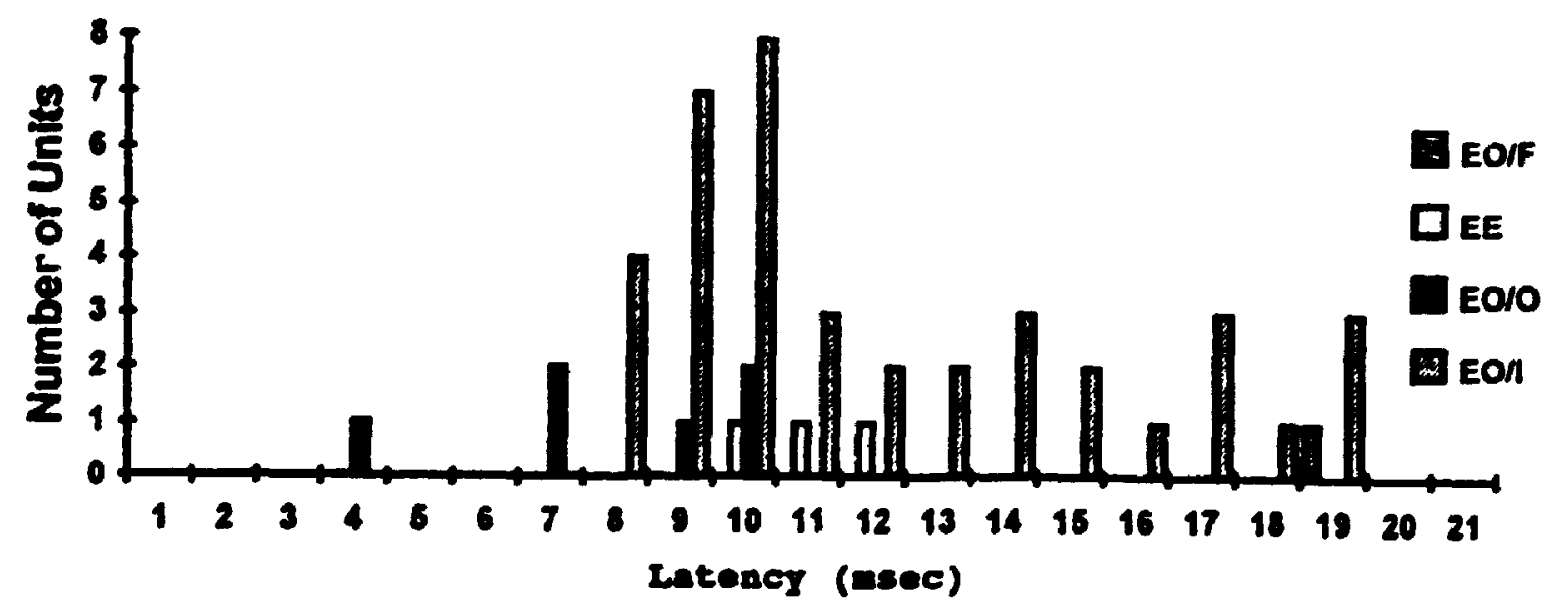


sample of sustained responses in DNLL (sample of 12), $33 \%$ were found to show some chopping, and for a sample of onset-pause-sustained responses, 16.76 were found to show chopping ( 1 in a sample of 6 ). Figure 12 (Appendix D), which gives post-stimulus histograms for different sound pressure levels and frequencies of tone burst stimulation for two well-isolated single units with onset chopper responses, illustrates the further point that a neuron did not necessarily show chopping for all sound pressure levels or frequencies of tone burst stimulation. As can be seen from Figure 12 (Appendix D), neuron BU56-2 showed an onset chopping response for highest sound pressure jevels at stimulating frequencies of $1.38 \mathrm{kHz}$ and $4.50 \mathrm{kHz}$, but with other stimulating frequencies $(2.27 \mathrm{kHz}, 3.32 \mathrm{kHz}, 1.77 \mathrm{kHz})$ chopping was not evident. Additionally, this neuron showed a sustained response, but only for highest sound pressure levels with stimulating frequencies of $1.38 \mathrm{kHz}$ and $4.50 \mathrm{kHz}$. Neuron BU52-1 also showed an onset chopper response (most evident with stimulating frequency 8.32 b.l?) wh: sil oriy occurred at high sound pressure levels for certain frequencies. Furthermore, with binaural stimulation the chopping response couid be eliminated since increasing ipsilateral sound pressure levei reduced the response to a single spike onset response for some interaural intensity differences. These cases illustrate that a failure to observe shopping does not preclude the possibility that chofping would rave been observed under different stimulus conditions.

\section{Distribution of Temporal Response Types in the Lemniscal Nuclei:}

Figures showing percentages of numbers of temporal response patterns recorded in lemniscal nuclei (Figure 6b, Table 1) show a 
predominance of onset responses in DNLL, in comparison with INLL and VNLI, which showed higher proportions of sustained responses. A composite reconstruction showing the distribution of temporal response patterns within the lemniscal nuclei shows some partial segregation of temporal response patterns within each lemniscal nucleus (Figure $5 \mathrm{c}$ ). in DNLL, more onset responses were found dorsally and medially, while sustained and onset-pause-sustained responses were usually found further ventrally. In INLL, although most neurons showed sustained responses, other types of responses (onset, onset-pause-sustained). when they occurred, were usually located near the borders of INLL in the same regions that binaurally sensitive neurons were found. The neurons in the binaurally sensitive medial region of VNLL also gave a mixture of temporal response patterns, which included some onset and onset-pause-sustained responses. Thus, there is a possibility that, across the lemniscal nuclei, onset or onset-pause-sustained responses are correlated with binaural sensitivity: this is a possibllity vindeh

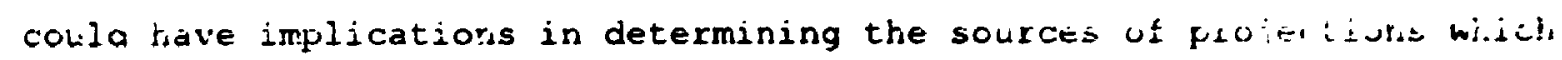
result in binaural sensitivity in the lemniscal nuclei.

\section{Dersomedial INLI:}

A dorsomedial region of INLL, close to the korder of DNLL and INLI, was fcund in several electrode track penetrations to show unusualiy high levels of spontaneous activity, and unusually high sensitivity (low th.resholds) to noise burst stimuli. One neuron which was localized in this region, exhibited an EE summation response, and showed an onset response which resembled typical DNiL orset resperises. but the response showed habituation (suppression of the response, with 
repeated presentations of the same tone burst stimulus).

\section{Paralemniscal zone and Regions Further Medial:}

The region medial to the lemniscal nuclei (paralemniscal zone) showed a predominance of onset responses. A significant fraction of paralemniscal neurons were found to show habituation to repeated presentations of pure-tone burst stimuli (40f of a sample of 10$)$ : in contrast, only one neuron showing habituation effects was found in any other lemniscal region in this study.

Temporal response patterns different from those found in DNII were found in regions extending as medial, or further medial than the paralemniscal zone, and included sustained spike trains which continued long after stimulus offset (many with poor stimulus-locking), onsetpause-sustained responses with variable length of pause lor gap in post-stimulus histogram where no responses occurred, and response after offset (some far medial responses are not shown in Figure 5c). Some of these newions were probably recorded from reticular formation nuclei.

\section{Latencies to First Response:}

DNLL:

Figure 10 shows a comparison of latencies to first spike response measured at $10 \mathrm{~dB}$ sound pressure level above inreshold for contralateral sound stimulation at characteristic frequency, for neurons in lemniscal regions. For these measurements, the threshold was operationally defined as the lowest sound pressure level at which a stamulus-locked response could be obtained. Responses from DNLL had characteristicaliy short latencies; mean latencies were 11.86 msec for 
Eiqure 10A-B. A. Distribution of latencies to first spike response. in response to pure tone bursts at characteristic frequency (CE), $10 \mathrm{~dB}$ above threshold, plotted as a function of CF, for neurons localized to DNLL, INLL, sagulura, or areas medial to DNLL and INLL (paralemniscal zone). B. Medial-lateral distribution of latencies in DNLL and surrounding structures. Latencies to first spike response, in response to pure tone bursts at CF, $10 \mathrm{~dB}$ above threshold, are plotted as a function of $\mathrm{CF}(\mathrm{kHz})$, for neurons localized to lateral DNLL, medial DNLL, central DNLL, sagulum (lateral to DNLL), or to areas immediately medial to DNLL (paralemiscal zone).

A.

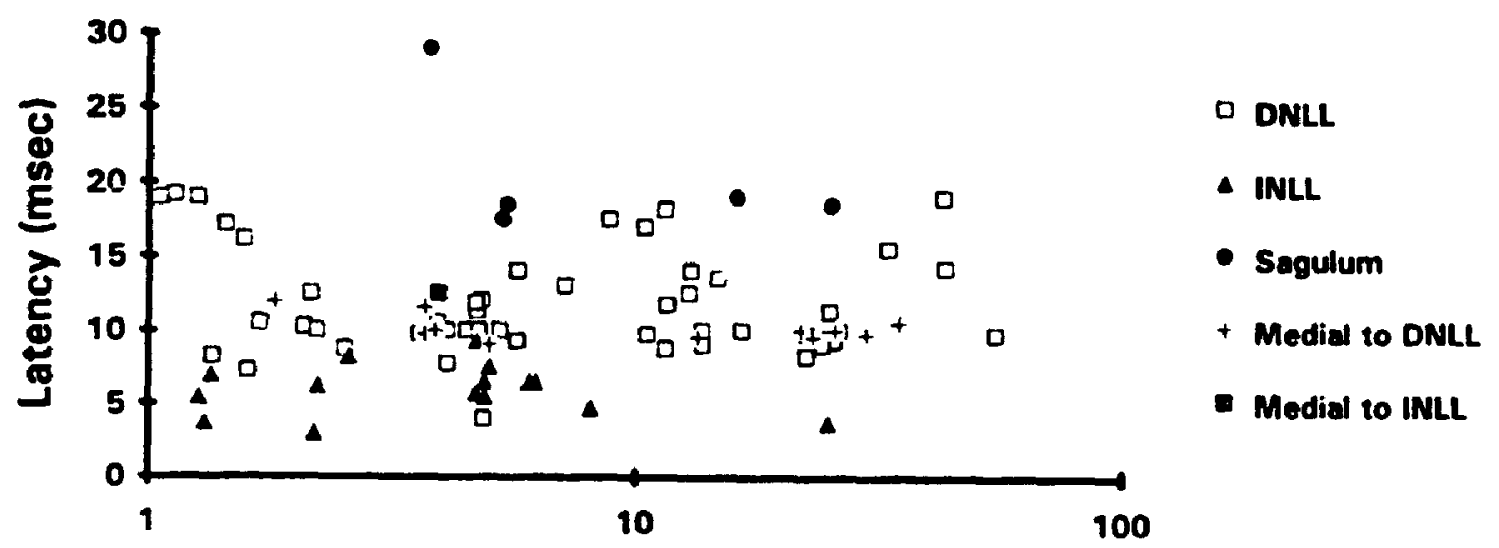

Characteristic Frequency (kHz)

B.

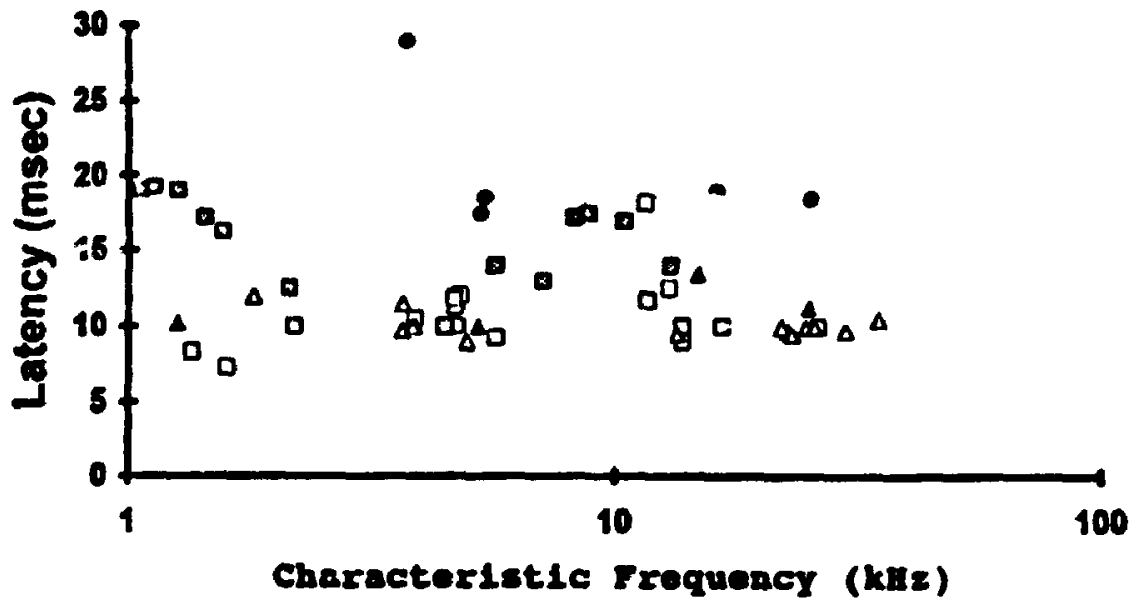

- Leteral DNLL

- Medial DNLL

- Central DNLL

- saguitum

- Medial to DNLL 
Figure 11. Paradoxical latency shift as illustrated by the responses of neuron BU27-2 ICF $=10.62 \mathrm{kHz}$ EO/I onset responsel t.o contralaterally presented pure tone bursts at characteristic frequency, for two different sound pressure levels. A. With the tone at $78 \mathrm{~dB}$, the first spike in the response occurs at a latency of approximately 9 msec, and is separated from subsequent spikes by gap or notch of several milliseconds. B. With sound pressure level increased to 88 $d b$, the unit no longer fires the first spike in the temporal response pattern of $(A)$. The latency is now that of the response following the notch, and is approximately 11 milliseconds. Stimulus duration was 110 milliseconds.

A.

8
4
0

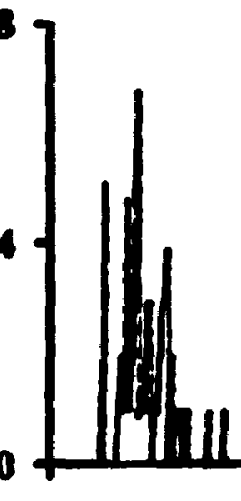

BU27-2 CF=10.62 kH2 $78 \mathrm{~dB}$ $N=67$

B.

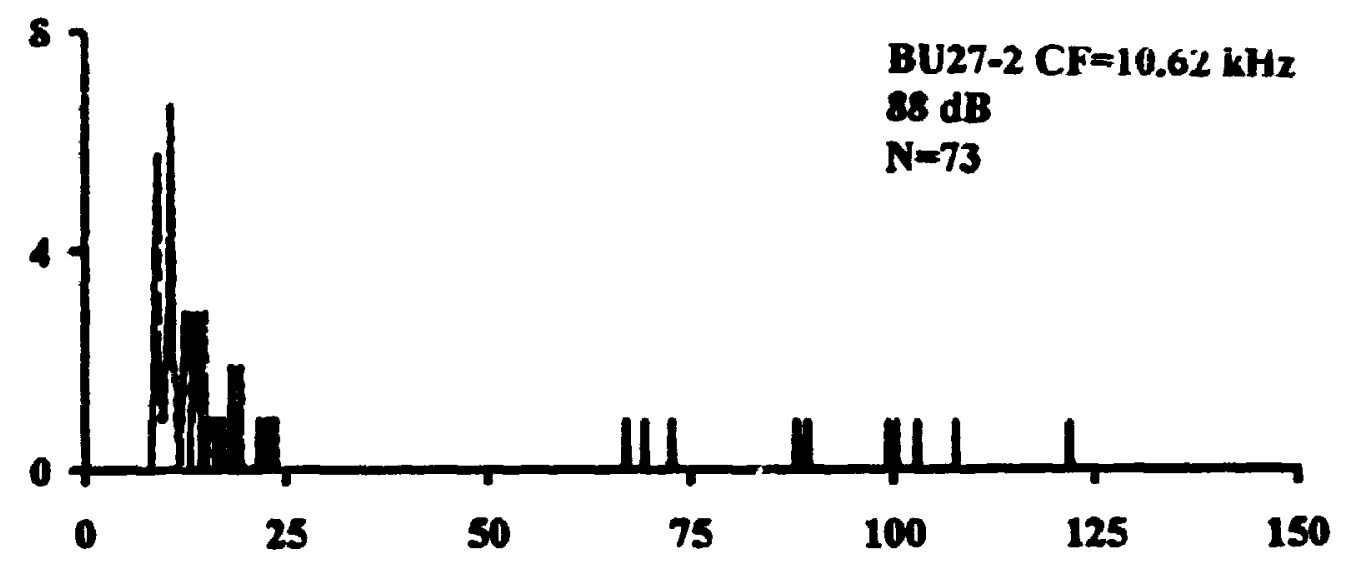


DNLL neurons (in a sample of 48 which included ail rostral and caudal levels), and 12.6 msec for central DNLL neurons (sample of 35. excluding rostral or caudal neurons) (Figure 10). Had the sound pressure level at which 508 of the maximum spike count occurred been used as the operational definition of threshold, the latencies to first. spike response for DNLI neurons would have been lower (mean latency 8.93 msec, sample of 35 ). The majority of DNLL neurons showed a monotonic decrease in latency as the contralateral sound pressure level was increased above threshold.

Neurons in the lateral part of DNLL had: newhat long latencies (mean latency of $16.32 \mathrm{msec}$ in a sample of 12 neurons). If the lateral DNLL neurons are not included in the sample, the mean latency for DNLL becomes somewhat shorter (mean of 10.37 msec, $N=36$ instead of 11.86 . $N=481$.

The neurons in DNLL showed a large spread in their distribution of latencles (Fıgure 9), with values of latencies ranging from 4.0 to 19 misec. juch a wide distribution of latercies might be expecteci wi the basis if the diversity of sources of projections to DNLL. Ar analysis of distribution of latencies for different tinaural response types of neurons suggests that EO/O neurons might have shorter latenczes than Eo/I neurons, but conclusions that can be drawn are $12 \mathrm{mited}$ by the small sample size of EO/O neurons.

\section{Sagulum:}

Sagulum neurons had a mean latency of $20.5 \mathrm{msec}$ (sample of $\mathrm{s}$ neurons, from case BU4t-R). The mean latency for sagulum relirons was longer than mean latencies for DNLL or lateral UNiL. Thus long 
latencies in sagulum distinguish it from it's bordering regions.

\section{INLL and VNLL:}

INLL and VNLL neurons showed latencies which were, on the average, shorter than those for DNLL neurons (mean latency of 5.98 msec for INLL neurons, sample of 15 , mean latency of 6.88 msec for VNLL, sample of 21 (Figure 10$)$.

\section{Paralemniscal zone:}

Areas medial to DNLL and INLL had mean latencies which were similar to those for DNLL, but showed longer latencies than those found in INLL Imedial to INLL, mean latency 12.0 msec with sample size of 2 ; medial to DNLL, mean latency $10.12 \mathrm{msec}$ with sample size of 13 ).

\section{Paradoxicai iatency Shift:}

For most neurons in the central auditory system, the first-spike latency becomes shorter as sound pressure level is increased. Constant latency neurons were an exception to this generalization found in the bat WNI (Covey and Casseday, 1991), and neurons that exhibit "paradoxical latency shift" show an even more extreme deviation from this rule (Covey, 1993). For these neurons, the latency to a monaural stimulus increases as sound pressure level increases. Neurons with paradoxical lateney shift were found to make up 14 : of 35 units tested in DNLL (Covey, 1993), although no such units were found in rostral 
DNLL (dorsal paralemniscal zone) (Covey, 1993), or in INLL or VNLL (Covey and Casseday, i991).

Although the present study did not systematically search for responses showing paradoxical latency shift. a few examples of increases in latency with increasing sound pressure level were found in DNLL (typical example in Figure 11). In all cases that were found, the latency increase at high sound pressure levels was due to suppression of an initial spike that was temporally separate from the later response. Thus these neurons had temporal response patterns which showed a short gap or notch lof a few milliseconds) following initial onset spikes; as sound pressure levels were increased, the jnitial onset spikes no longer occurred so that the gap was no longer apparent (Figure 12).

\section{Rate Intensity Functions:}

EXI:E:

Most rate intensity functions measured at charactels $s^{*}$ ic freg.ten: for relrons in DNLL showed a monotonic increase in firing rate with increases in contralateral sound pressure level. A minority $123.0 \%$, sample of 22 ) of rate intensity functions were non-monotunc, and showed a decrease in firing rate of at least 10 per cent. Figure $1 \%$ A censtrasts typicel monotonic and non-monotonie funcelcris.

For some monotonic functions, the firing rate showed no further increases as sound prcsizure level was increased bejond a celtan jevel. while $2 n$ other cases, increases in sound pressure ieve. resulied in increases in firing rate even for sound pressure levels 49 dB above 

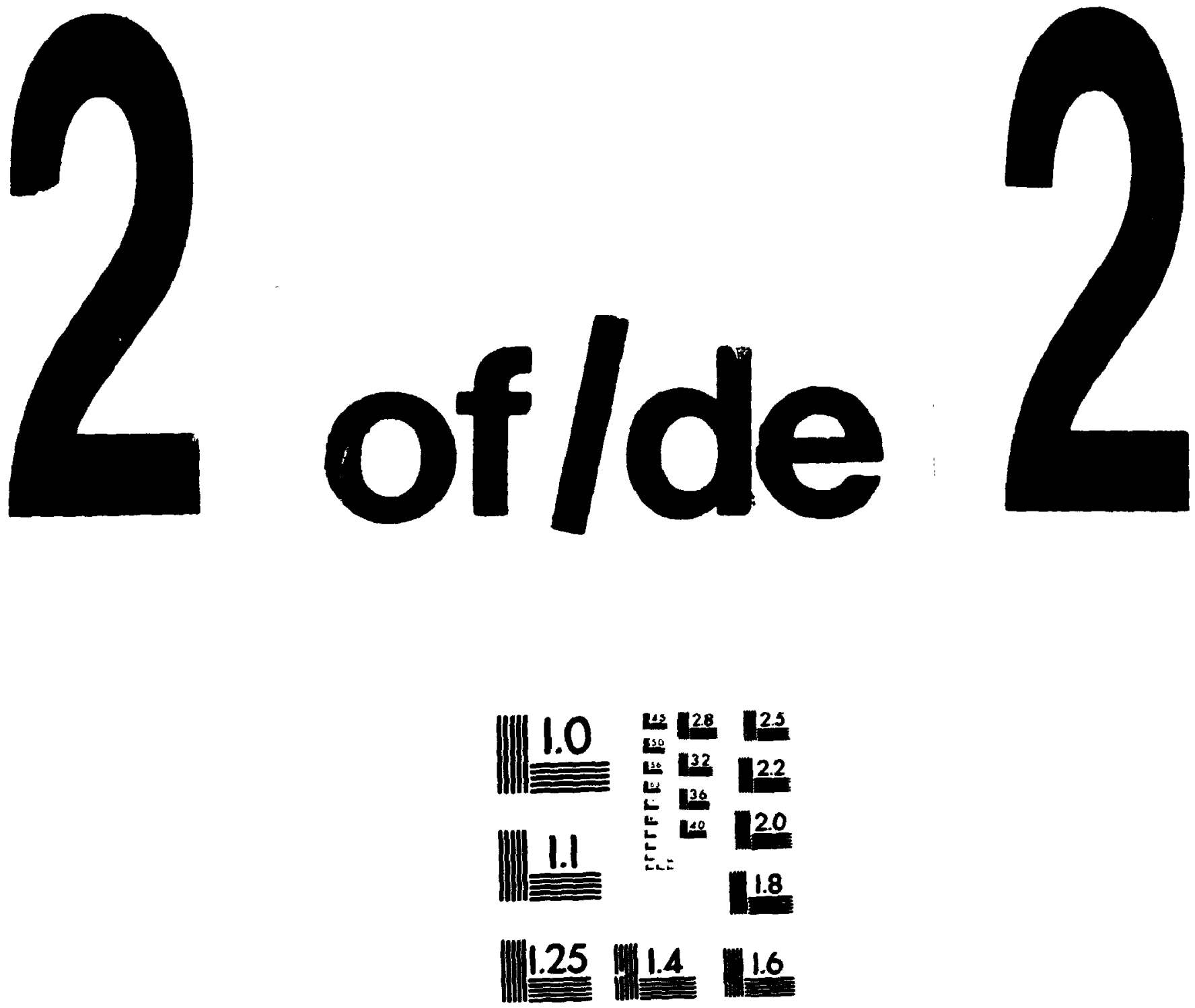

MICROCOPY RESOLUTION TEST CHART

NATIONAL BUREAU OF STANDARDS

STANOARD REF ERENCE MATERIAL 1010a

(ANSI and ISO TEST CHART NO 2 ) 
Figure 12. Rate intensity functions for single units in DNLL (A) ahow that both monotonic and non-monotonic relationships between sound level and firing rate were found. Rate intensity functions in (B) were monotoric functions for neurons dorsal to the main division of DNLL. To allow a comparison among units with different firing rates. responses are normalized to the maximal evoked firing rate, and plotted as percentage of maximum response. In (C), the rate intensity functions measured at different frequencies of tone burst stimulation are shown for BU56-2, CF $=4.5 \mathrm{kHz}$, an onset chopper (with some sustained respondingl in central DNLL. This plot illustrates that lower thresholds for responding occur sur frequencies close to the characteristic frequency.

A.
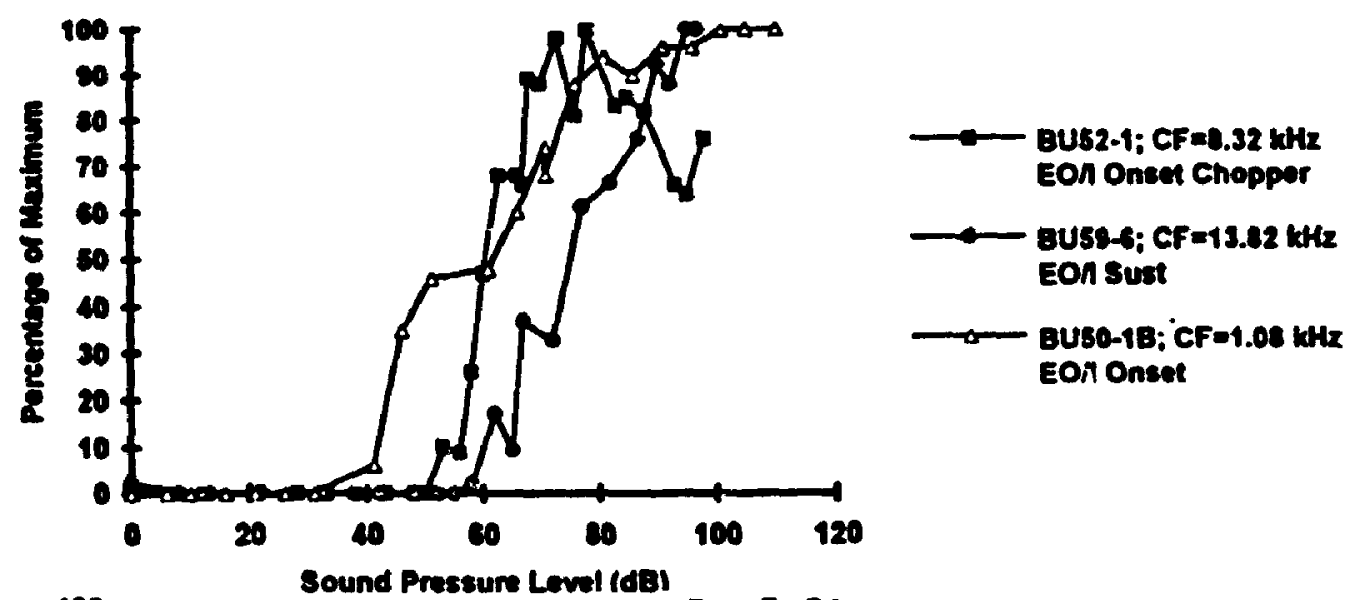

B.

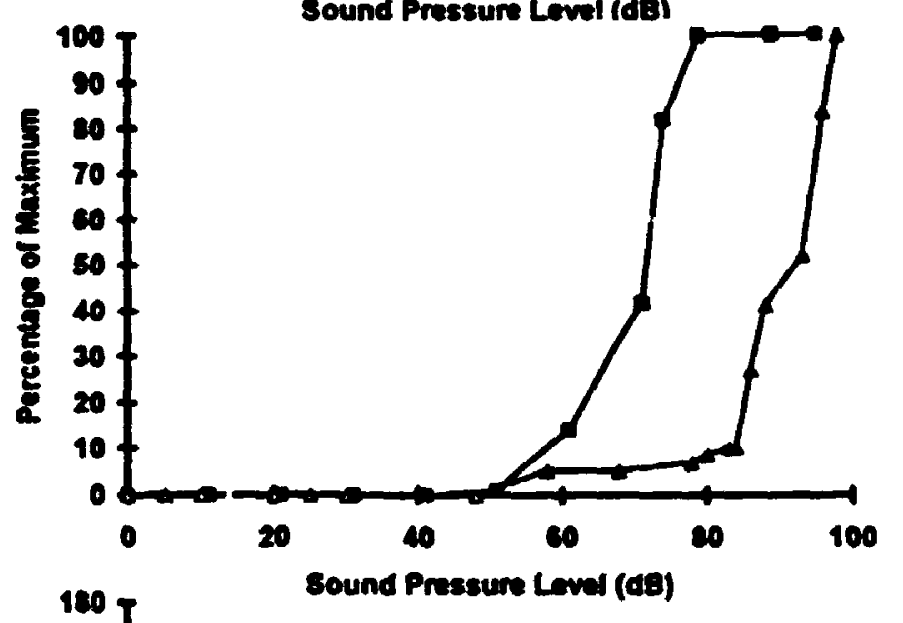

US5-1; CFe1.04 kHz EOn mixad sust

C.

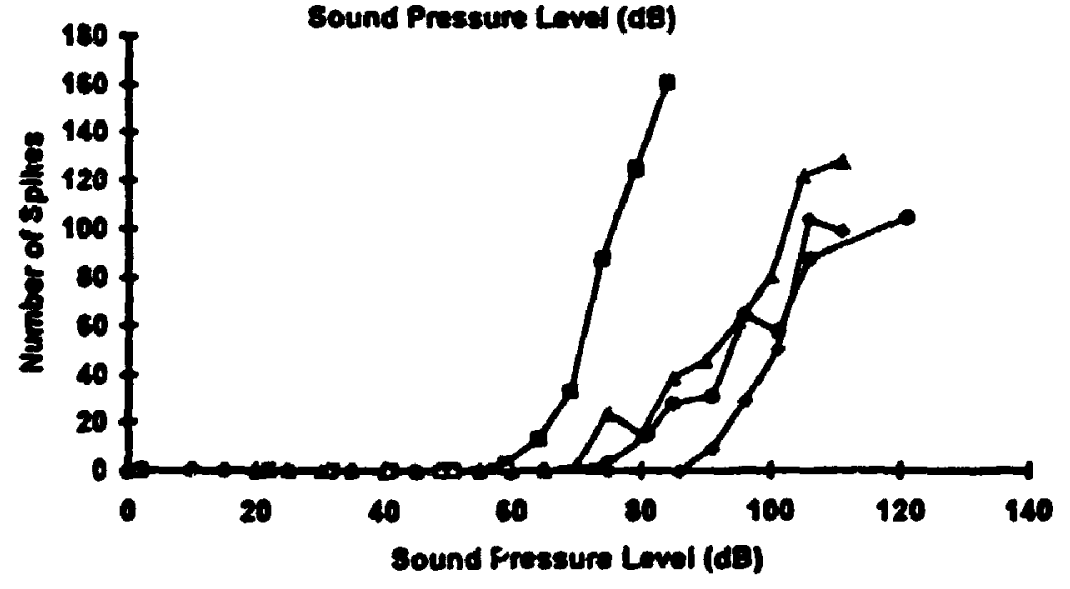

1.31 kits $-1.77 \mathrm{mHz}$ $2.27 \mathrm{wh}$ $\rightarrow-4$ sink 
threshold. DNLL neurons with onset responses, on average, had rate intensity functions with lower dynamic ranges, and would respond with maximal fixing rate at 10-20 dB above threshold (e.g.. BU50-1B CF $=$ 1.0B kHz, Figure 12a). The proportion of onset neurons for which increases of sound level of more than $20 \mathrm{~dB}$ above threshold did not cause any further increases in firing rate (66.78 of sample of 12 ) was greater than the $r$ dortion for sustained (25.08 of a sample of 4$)$ or onset-pause-sustain, neurons (37.58 of a sample of 8 ). Figure 12-A contrasts the difference in dynamic range of rate intensity functions for typica? onset and sustained neurons.

\section{Other Lemniscal Regions:}

While samples of rate intensity functions obtained from regions other than DNLL were small, both non-monotonic and monotonic functions were obtained in INLL (sample of 1 non-monotonic, 2 monotonic functions, and 2 monotonic rate intensity functions were obtained

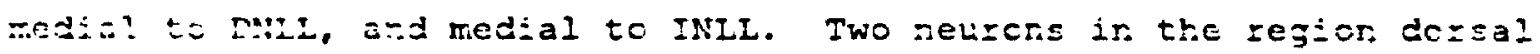
to the maja division of DNLI (BU55-1 and BU55-2, Figure 12) had rate intensity functions similar to most found in DNII, with no evidence of non-monotonic behaviour.

\section{Interaural Intensity Difference (IID) Functions:}

DNLI:

Typical interaural intensity difference (IID) functions measured from EO/I neurons in central DNLL with different temporal response patlerns (Figure 13), showed a decrease in neuronal firing rate as ipsilateral sound pressure level was increased. With highest levels of 
Figure 13A-I. Interaural intensity difference (IID) curves from EO/I units with different temporal response patterns, and varying rates of spontaneous activity, in central DNLL (no rostral or caudal neurons included), INLL, and dorsal to DNLL. Percentage of maximum spikt rate plotted against IID, with contralateral subtracted from ipsilateral sound pressure level (dB SPL). Rate of spontaneous activity, spike rate for contralateral sound only (disconnected from curve, it high positive or negative IID values, respectively), and characteristic frequency (CF) are shown. A. DNLL onset choppers; no spontaneous. B. DNLI onset non-choppers (1 spike at onset); no spontaneous. C. DNLL onset non-choppers (1 spike at onset); spontaneous activity. D. DNLL onset-pause-sustained with chopping; varying rates of spontaneous activity (including a rate of zero, BU18-3). E. DNLL onset-pausesustained, no evidence of chopping; spontaneous activity. F. DNLL sustained, no evidence of chopping: low ratex of spontaneous. G. INLL onset chopppers: no spontaneous. H. INLL onset chopper with sustained component; no spontarieous. I. Dorsal to DNLL, sustained EO/I mixed (BU55-1) and EO/F (BYS $5-2)$.

A.
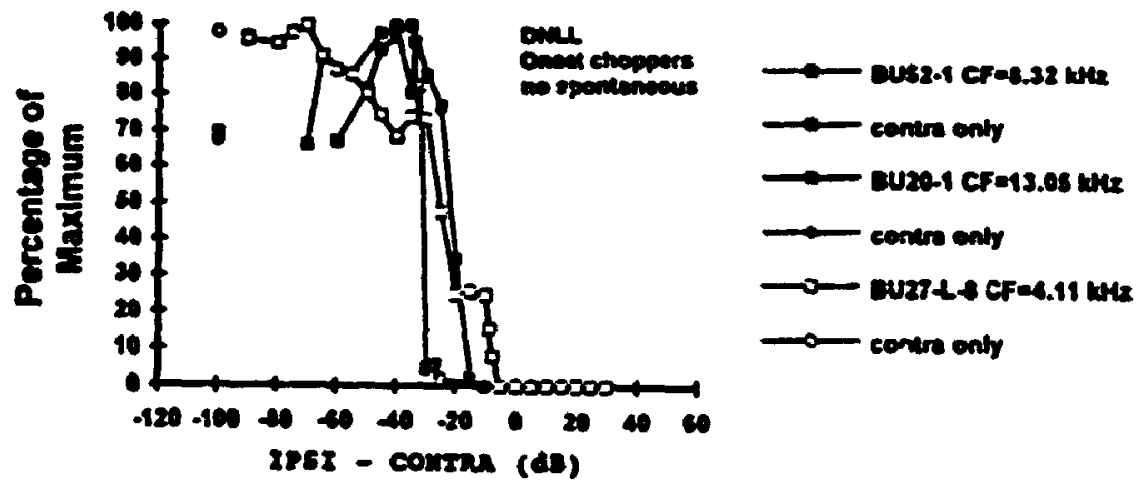

B.
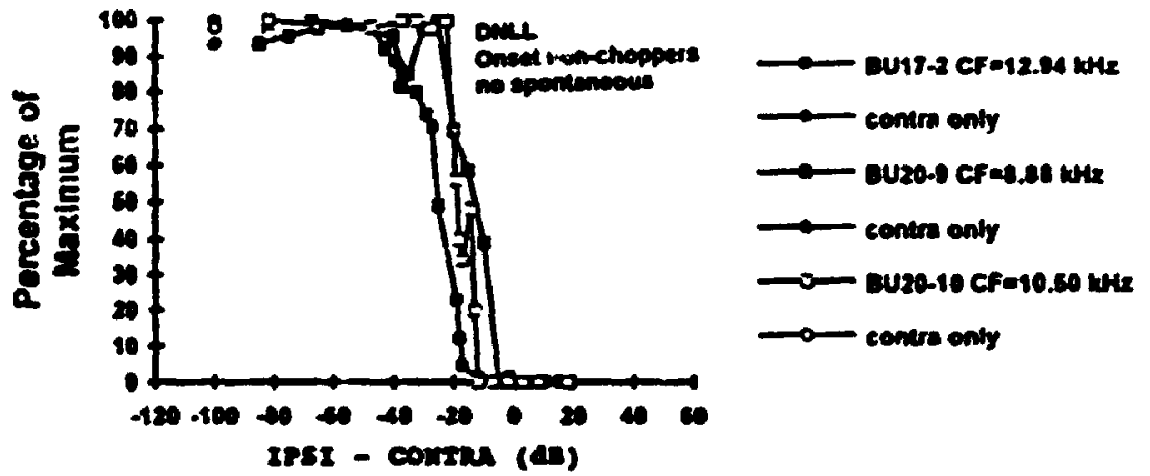

c.
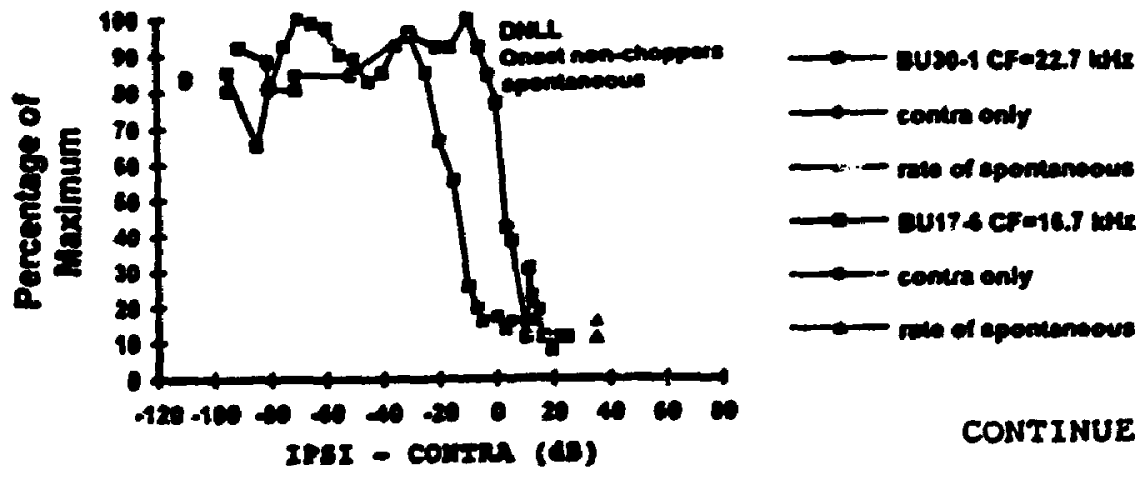

CONTINUED OVER 
D.
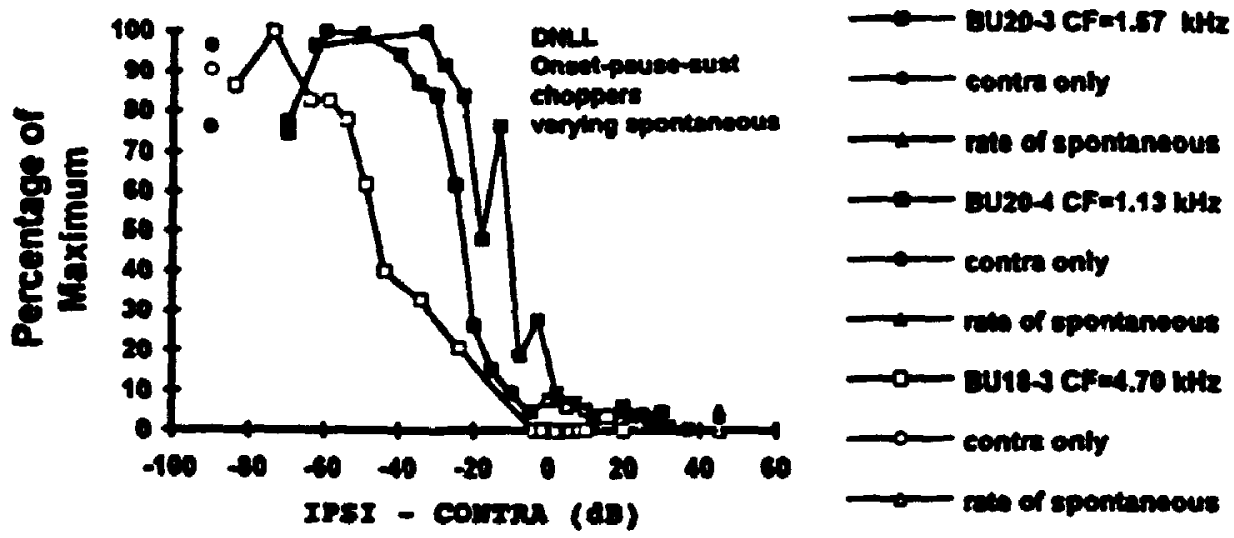

$E$
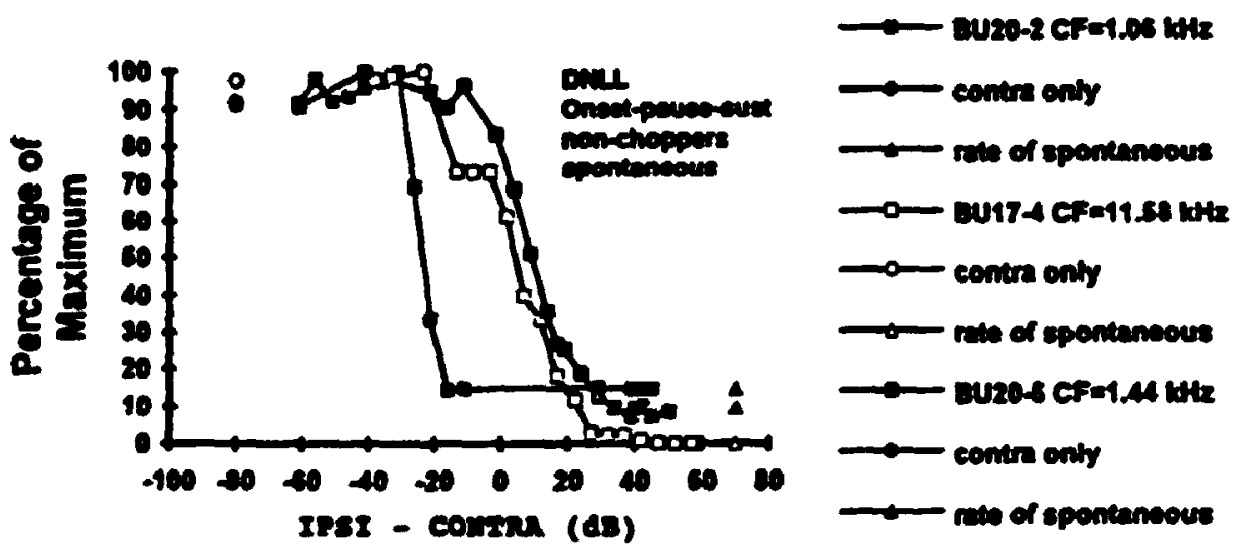

$F$
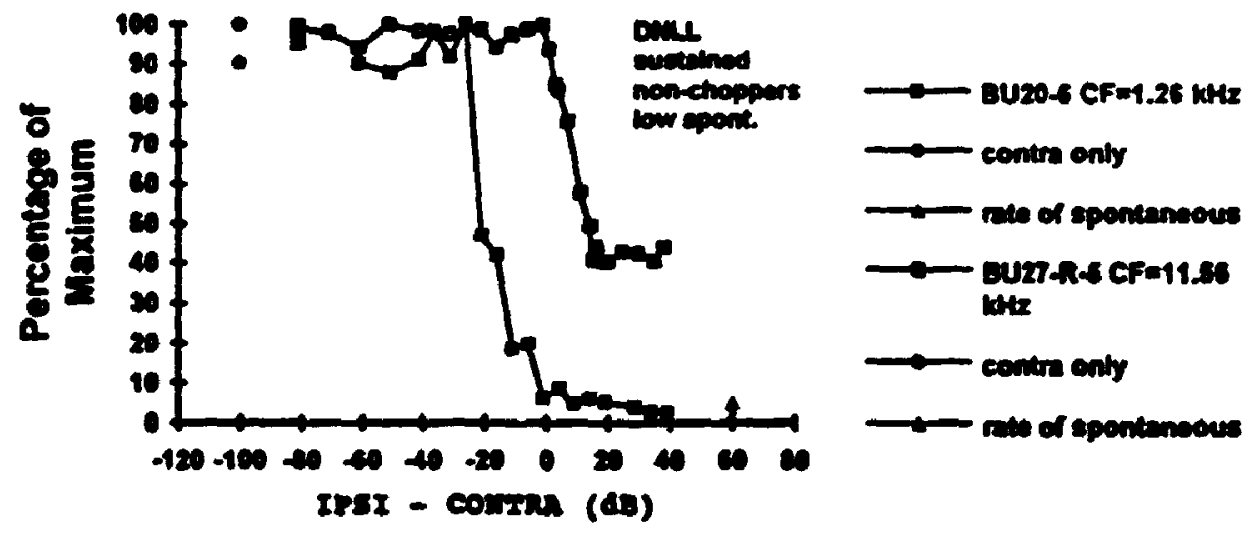

Figure 13. (continued) 
G.

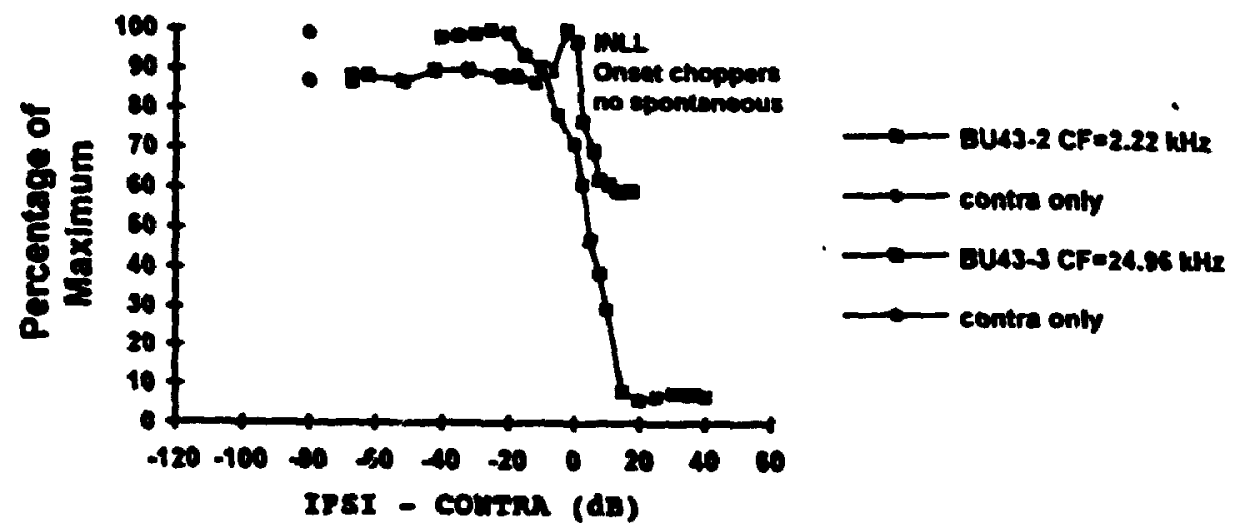

H.

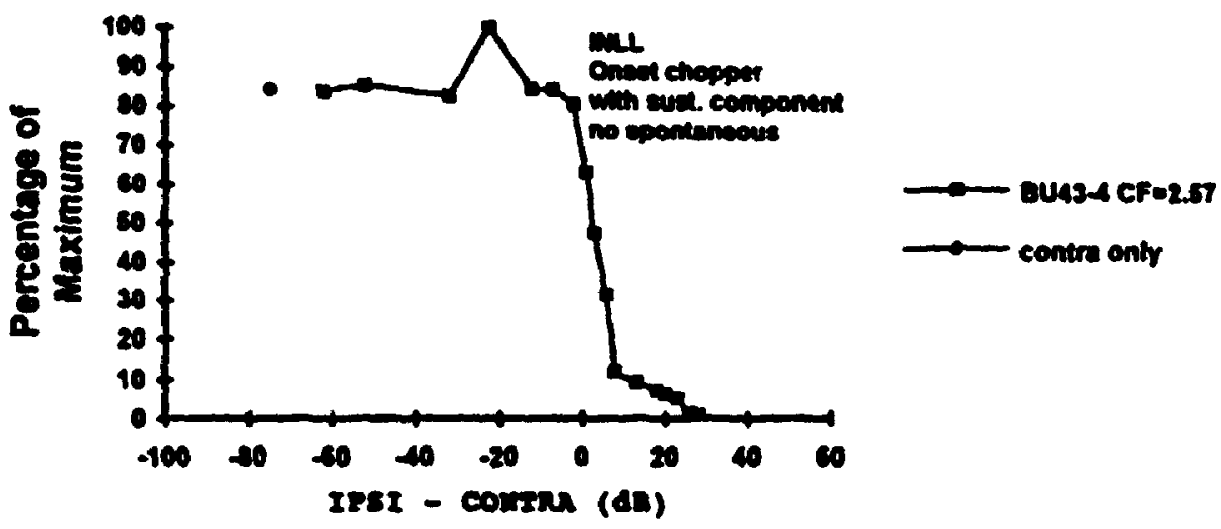

I.

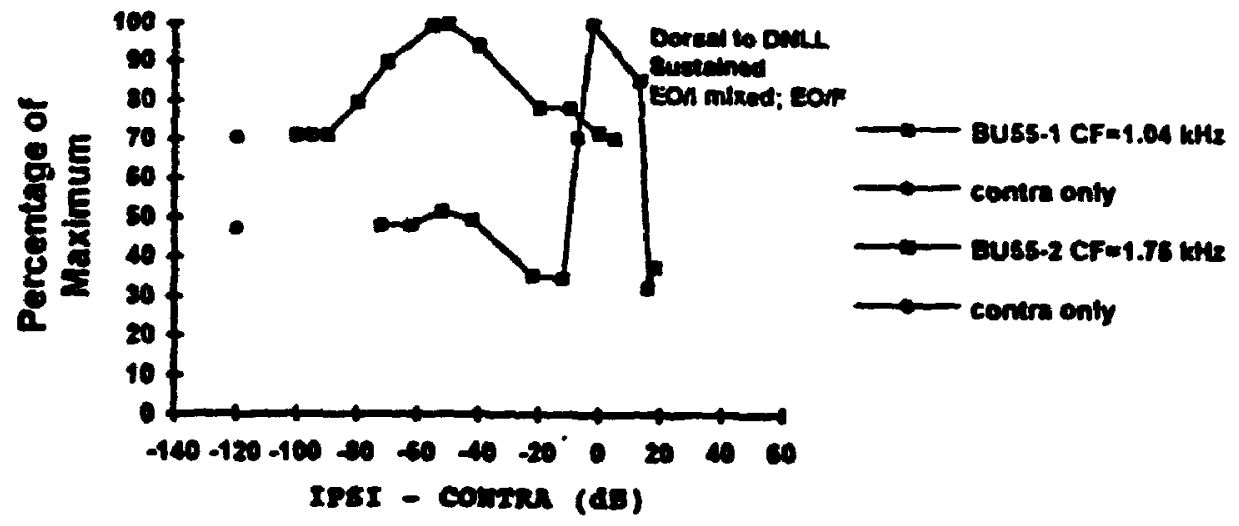

Figure 13. (continued) 
ipsilateral sound pressure level, the neuronal firing rate was reduced to zero; for neurons with spontaneous activity, the neuronal firing rate was reduced to the rate of spontaneous activity. These IID functions measured from DNLL reurons resemble IID functions previously measured from the inferior colliculus in the rat.

The majority of IID functions measured from neurons in central DNLL were monotonic, and no obvious differences were seen between curves obtained from neurons with different temporal response types. However, for some neurons, a comparison of the response to contralateral sound only, with the rest of the IID function, showed some facilitation as sound pressure level in the ipsilateral ear was increased, although as ipsilateral sound pressure level was increased further, the firing rate of the neuron decreased to zero (Figure 13: units BU52-1, BU20-1, BU20-4). These neurons could be termed EO/I mixed neurons, since a mixture of facllitation and suppression was shown.

A sample of single units in the central binaural region of DNLI, fil wistl obsestations on tenporal resporse patterns at different frequencies of stimulation permitted an unequivocal classiflation of temporal response type (onset, onset-chopper, sustained, sustainedslow-chopping, onset-pause-sustained, or onset-pause-sustained with some choppingl, were sibjected to further analysis of IID functions. IID functions measured at characteristic frequercy were analyzed for possible non-monotonic behaviour. It was found that while any given temporal response pattern colild be associated with IID functions with either monotonic or non-monotonic behaviour, all non-monotonic IID functions were measured from neurons exhibiting chopping patterns (either onset-chopper or onset-pause-sustained with chopping). of the 
22 DNLL neurons subjected to this analysis, 3 (13.6) showed nonmonotonic IID functions, while the remaining 19 neurons $(86.4 n)$ showed no evidence of non-monotonic behaviour. The 3 neurons evidencing nonmonotonic behaviour included 2 onset-choppers and 1 neuron with an onset-pause-sustained (with chopping) response. All of the neurons which showed non-chopping oriet (single spike at onset) responses (sample of 7), sustained (non-chopping) responses (sample of 4), or onset-pause-sustained (non-chopping) responses (sample of 4 ) evidenced only monotonic IID functions.

While most EO/I neurons recorded in DNLL showed complete suppression of the response for high enough sound pressure levels at the ipsilateral ear, for a few neurons exhibiting sustained responses, the onset component of the response was not suppressed at any sound pressure level. The typical shape of the IID function for such a neuron is shown in Figure 13-F (neuron BU27-R-5): such neurons were senistive to a limited range of interaural intensity differences. All

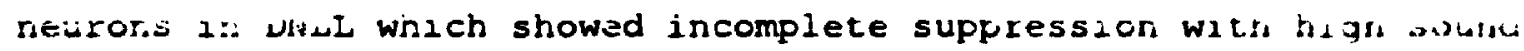
pressure Levels at the ipsilateral ear were iocated in vertral IML.

Regions Dorsal to the Main Subdivision of DNLL:

Figure 13-I illustrates that IID functions measured from neurons in the region dersal to the main subdivision of DNLi demonstrated very different binaural response properties from neurons in central DNLL. The EO/E neuron (BU55-2) exhibited facilitation, since it showed an increase in response rate above that zesulting from contralateral (monaural; stimulation, once ipsilateral stimulation was appliea. However, the facilitation resulting from binaural stimulation only 
occurred for a certain range of IID values (approximately IID $=-90$ to -30). No suppression of the response rate below the response rate for contralateral (monaural) stimulation occurred for any ipsilateral sound pressure levels, and for this reason, the neuron was classified as an EO/F neuron.

In contrast, neuron BU55-1 (EO/I mixed) exhibited both a reduction and an increase in response rate, in comparison to the response rate for contralateral stimulation, for different interaural intensity differences. Maximal firing rates occurred for IID values ranging approximately from -15 to +15 (IID values for a sound source close to the midline). The reduction of firing rate below that for contralateral stimulation occurred for IID values immediately on either side of this certral facilitatory region li.e., IID $=-15$ to -25 and IID $=+151$; it seems to likely that such suppression would increase the neuron's sensitivity in distinguishing between IID values inside the facilitalsy response region for the neuron, and those outside the fat:ilabury response region.

In summary, responses measured from the region dorsal to the main sutdivision of DNLL showed IID functions markedly different from the IID functions found in central DNLL.

\section{INLIs and VNLL:}

The majority of neurons with EO/I suppression responses recorded from binaural areas of INLL and VNLL had IID functions similar to those found in central DNLL, with both monotonic and non-monotonic IID functions present.

However, a further analysis of interaural intensity difference 
functions revealed that a large proportion of neurons exhibited incomplete suppression of neuronal responses with increased ipsilateral sound pressure levels. Figure 13-G illustrates two examples of IID functions from INLL neurons for which the spike counts did not decrease to zero with increased ipsilateral sound pressure levels (BU43-2, BU433 in Figure 131.

Neurons in medial and ventral INLL from two electrode tracks (BU43. BU50), included a high proportion of neurons exhibiting incomplete suppression ( 408 of a sample of 5$)$. Both neurons exhibiting incomplete suppression were onset choppers.

In VNiL, a medial region (case BU45) or dorsal region (case BU59) showed a similar proportion $(42.98$ of a sample of 7$)$ of neurons exhibiting incomplete suppression of responses with ipsilateral stimulation. Two different mechanisms for incomplete suppression in ViLL neurons were found: sustained responses were transformed into onset respunses with increased ipsilateral solnd pressure level, while enset-pause-sustained responses showed increases in latency and decreased spike counts with increases in ipsilateral sound pressure level (but the temporal pattern of the response still included a sustained component .

\section{Erequency Turing:}

DNLL, Rostral LNLL, and Areas Dorsal to DNLL:

Representative frequensy tuning curves for neurons in central DNLL (a sample of 18 neurons, which excludes rostral or caudal neurons), rostral Divil (cases BU60, BU61), and in areas dorsal to DHLL are shown in Figures 14A, 14B, and 14D. Most tuning curves from central DiflL 
Fiqure 14. Representative tuning curves from single units showing threshold (dB SPL) plotted as a function of frequency of pure tone burst stimulation. A. Central DNLL. B. Rostral DNLI. C. INLL. D. Dorsal to DNLL.

A.
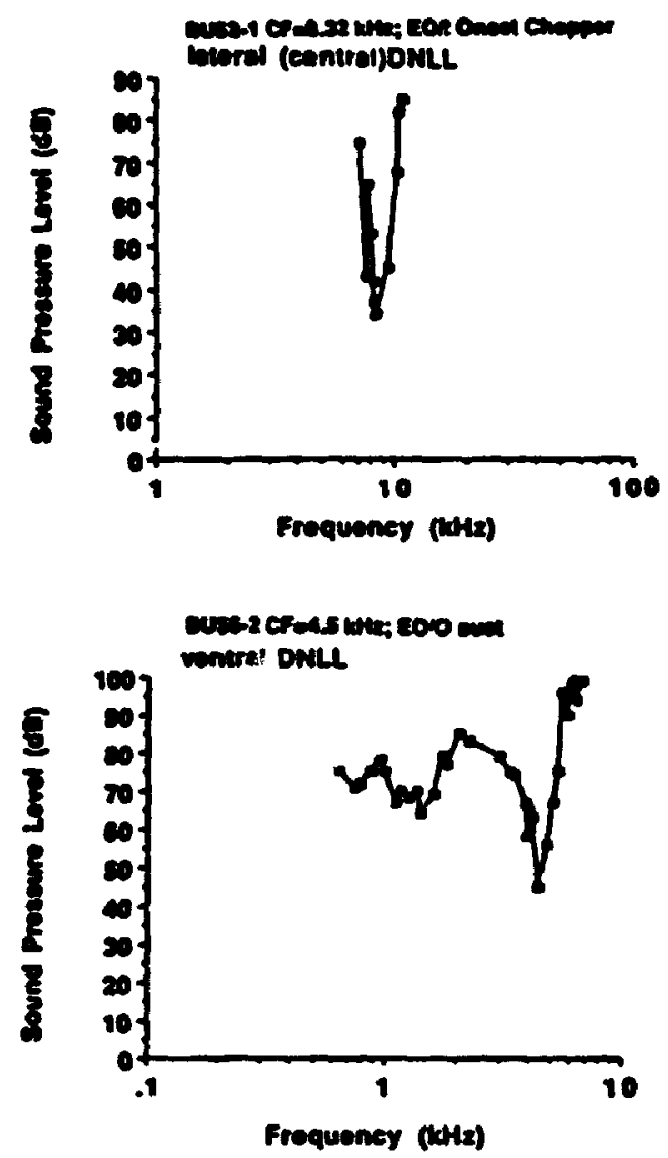

B.

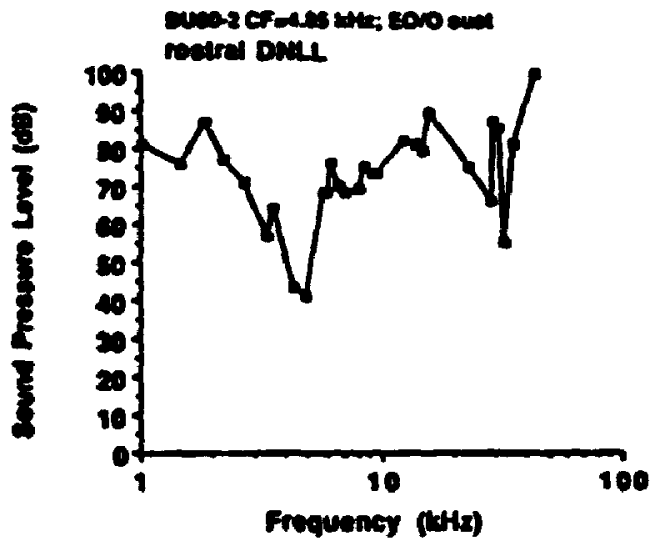

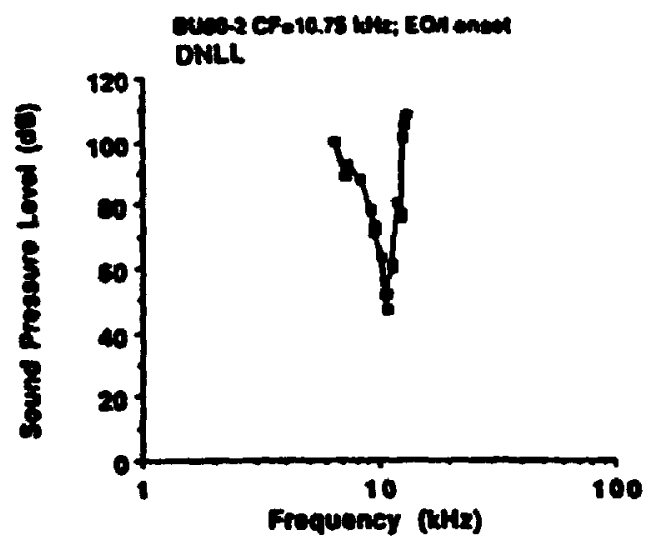

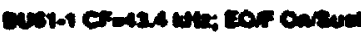
reatres Dans

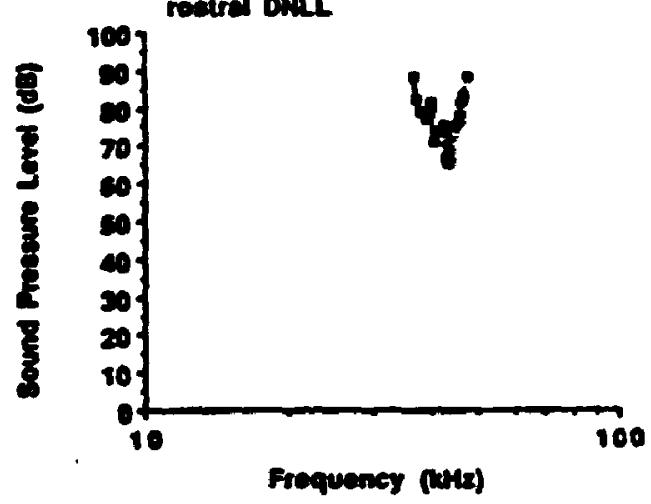

CONTINUED 
Figure 14. Representative tuning curves from single units showing threshold (dB SPL) plotted as a function of freguency of pure tone burst stimulation. A. Central DNLL. B. Rostral DNLL. C. INLL. Dorsa? to DNLL.

B.

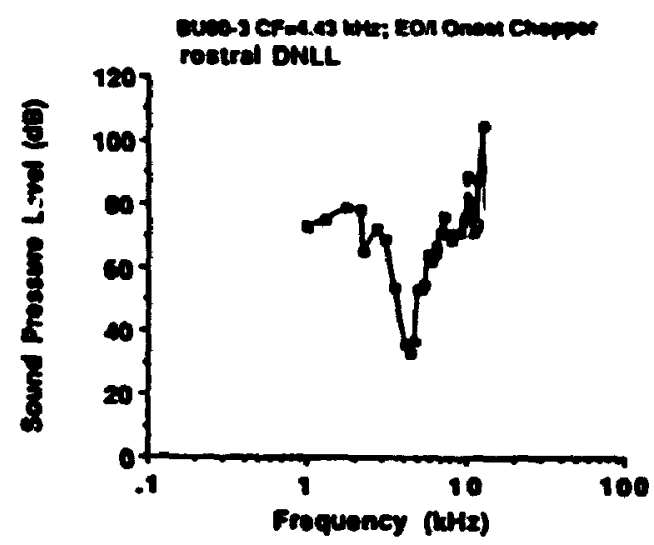

C.
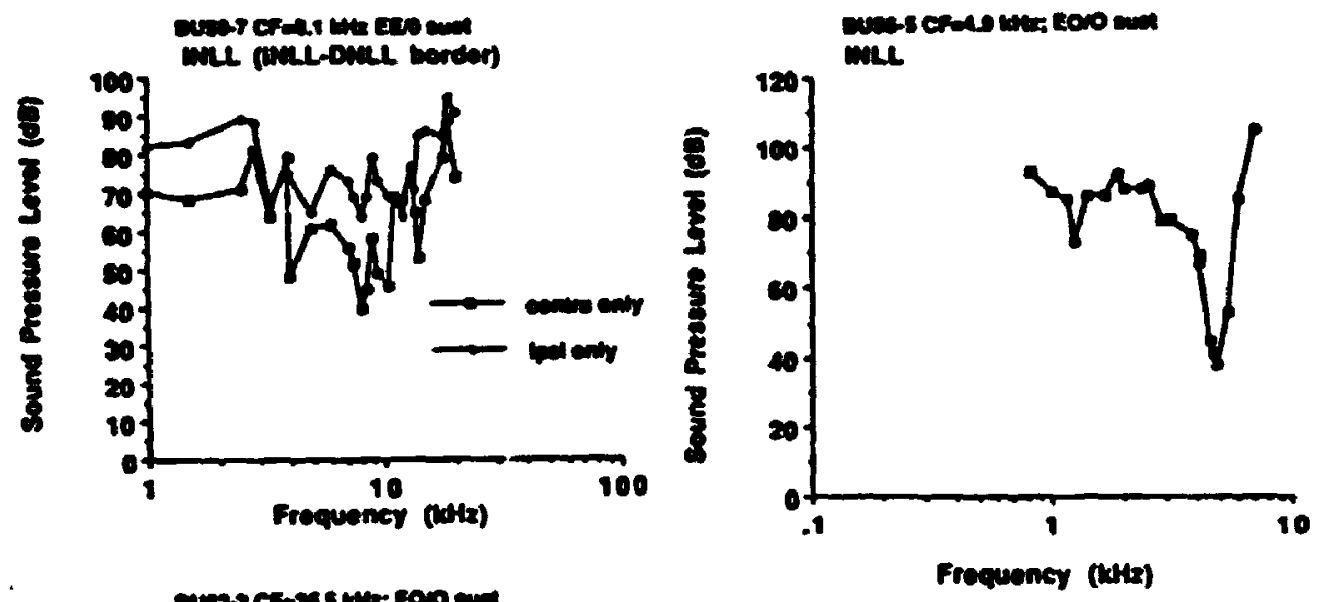

D.

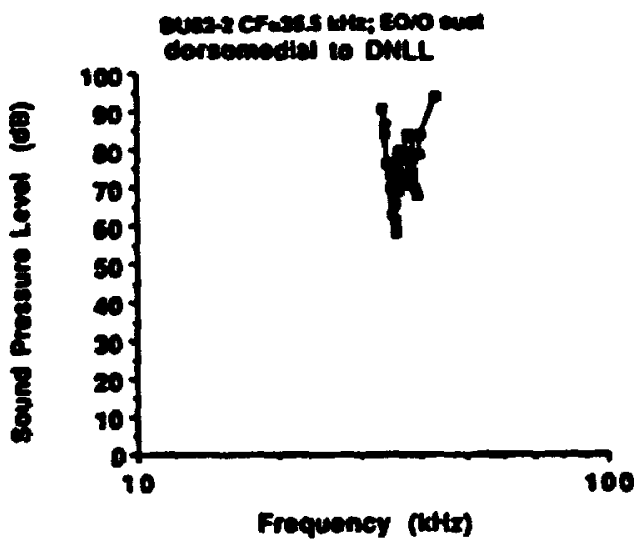

Figure 14. (continued) 
were $V$-shaped, and symmetrical or with low-frequency tails (showing that neurons would respond to a range of frequencies lower than the characteristic frequency, for high enough sound pressure levels). Although frequency tuning was sharp, neurons were not, on average, as sharply tuned as those found in the central nucleus of the IC, for which mean $Q(10 \mathrm{~dB})$ values (characteristic frequency, divided by the bandwidth of the tuning curve $10 \mathrm{~dB}$ above threshold) are typically greater than 10 (e.g. Aitkin et al.. 1975; Merzenich and Reid, 1974; Rose et al., 1963). The sample of 12 neurons in central DNLL had a mean $Q(10 \mathrm{~dB})$ value of 7.02 .

The small sample of 3 neurons from rostral DNLL showed somewhat broad tuning for low sound pressure levels (mean $Q(10 \mathrm{~dB})=5.29$ ), but for highest sound pressure levels, tuning was considerably broader than that of central DNLL neurons. For sound pressure levels $40 \mathrm{~dB}$ above threshold, the mean $Q(40 \mathrm{da})$ value of 0.61 for rostral DNLL neurons was consicieaily smallex than that for central DNiL neurons $: Q(40 \mathrm{~dB})=$

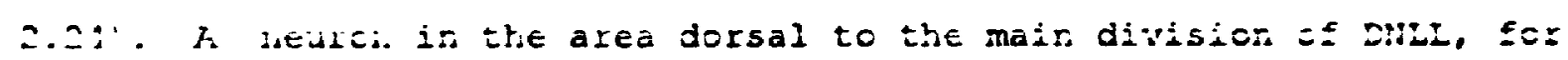
which frequency tuning was characterized, showed very sharp tuning at low sound pressure levels $(Q(10 \mathrm{~dB})=27.2$, but much broader tuning for higher sound pressure levels $(Q(40 \mathrm{~dB})=3.0)$.

Neurons in DNLL showing EE sumnation responses typically had a lower threshold for contralateral sound stimulation, than for ipsilatezal stimulation ( 898 of sample of 9 neurons in DNLL); this was also shown by tuning curves assessed contralaterully and ipsilaterally. Figuze 14-C gives an example of contralateral and ipsilateral tuning curves, for a neuron with an EE response. Like all tuning curves measured in DNLL for EE neurons (sample of 2), characteristic frequency 
and shape of tuning function was the same for stimulation of either ear.

INLL:

Figure 14-C illustrates that tuning curves measured from INLL (sample of 5) had similar shapes to those measured in DNLL, and could be V-shaped and symmetrical, or have low-frequency tails. The mean $Q(10 \mathrm{~dB})$ value of 5.8 was also similar to that for central DNLL neurons. 


\section{Discussion}

DNLL:

Responses from the DNLL of the rat were characterized by a large proportion of hinaural neurons (mainly EO/I responses), short latencies, somewhat narrow frequency tuning, and a relatively high percentage of onset responses. The high proportion of EO/I responses is in accord with previous studies in the bat (Covey and Casseday, 1986; 1991; Covey, 1993), and cat (Aitkin and Brugge, 1970).

Proportions of binaural response types found in the present study were similar to those found in the bat (Covey, 1993). Aitkin and Brugge (1970) did not clarify how many of theix binaurally sensitive neurons were sensitive to interaural timing differences (ITDs), or whether any of their sample of neurons would be excited by either ipsilateral or contralateral monaural stimulation (termed EE neurons in the present study). This makes it difficult to make an exact comparison of present resuits to this study.

S-nanial suppression (EO/I) responses were found to be present in the righest proportions within the central region of DNLL, where comissural fibers project to DNLL from the opposite DNLL. This suggests that it may be the neurons having EO/I suppression responses which project to the opposite DNLL or have projections from the opposite DNLL. It seems likely from this result that at least some of the commissural projections between the two DNLLs involve EO/I neurons. No clear linear frequency progression was found in DNLL, but this does not rule out the possibility of some other tonotopic organization. In some electrode penetrations, higher frequencies were located more ventrally; this is a similar result to those found in previous 
physiological studies (Covey, 1993; Brugge et al., 1970). Some rapid changes or reversals in characteristic frequency sometimes occurred along a given electrode track; some rapid transitions from lower (<6 $\mathrm{kHz})$ to higher $(>8 \mathrm{kHz})$ characteristic frequencies (e.g. Figure 4 , cases BU46-I, BU41, BU43) within DNLL were found. iuch rapid transitions in $C F$, as well as occasional reversals, were also found in previous studies (Covey and Casseday, 1986; 1991; Aitkin and Brugge, 19701. In some electrode tracks, high frequencies were found dorsally and laterally within DNLL. It might be argued that these results would be compatible with an "onion-like" tonotopic organization (Saldana, 19931, in which high frequencies are located around the outs ide borders of DNLi, or that results were compatible with the results of Friauf (1992) or Preuß (1991). However, no orderly progressions of high frequencies to lower frequencies, with a reversal to high frequencies were found, so no positive support for this model was obtained.

The physiological results of the present study and previous studies

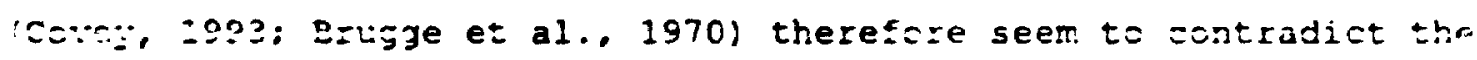
anatomical evidence suggesting a tonotopic organization within DNLL with higher frequencies represented in the border regions of DNLL (Saldana, 1993: Preuß, 1991; Friauf, 1992, Caicedo and Herbert, 1993). It is possible that the small size of the nucleus, small number of cells, and scattered distribution of cells presents difficulties in determining the tonotopic organization of the DNLL electrophysiologically. In addition, the laminae which may form the neural substrate for the frequency organization in Dill may be only one neu: on thick (Caicedo and Herbert, 1993), and may curve a:sund to form a complicated three-dimensional organization, which would not be 
evident from dorsoventral electrode penetrations (Sa)dana, 1993). It has also been claimed that the frequency organization in DNLL changes along the rostral-caudal axis (Saldana, 1993).

Interaural intensity difference functions from EO/I neurons in DNLL resembled those found previously in the inferior colliculus, raising questions as to why such binaural sensitivity seems to be duplicated at different levels within the avtitory system. A small proportion of IID functions in DNLL were found to be non-monotonic, and these nonmonotonic functions seemed to be associated with chopping responses (onset or onset-pause-sustained responses with chopping). However, a failure $t$ u observe non-monotonic behaviour in a response does not demonstrate that the neuron would not exhibit non-monotonic behaviour under other circumstances, and thus this result does not necessarily lead us to conclude that non-choppers do not show non-monotonic behaviour. A small proportion of rate intensity functions in DNLI were aisc found to exhibit non-monotonic behaviour. In a previous stuidy of

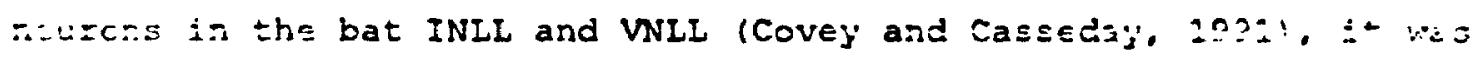
tound that all onset units had monotonic rate level functzors, while the majority (79t of 45 units) of sustained responses showed morotonic behaviour, and over half $(548)$ of choppers had non-monotonic rate level functions; it was found that differences related more to respcrse type than to the division of INLL, or VNLL in which the neuron was located (Covey and Casseday, 1991). Since these results are very similar to those found in the present study of DNLL neurons, this could offer the possibility that neurons with similar temporal patterns locateo in the different lenniscal nuclei may share some binaural response characteristics, and possibly have some ascending projections in 
common.

The non-monotonic IID functions in DNLL did not show as great a facilitation (when operationally defined as percentage increase in spike rate above the rate for contralateral sound only) as that seen for many mixed responses in the inferior colliculus. However, the level of the lemniscal nuclei (DNLL, INLL or VNLL) seems to be the first level in the ascending auditory system at which non-monotonic behaviour has been seen.

Several neurons in DNLL exhibited paraoiuxical latency shift, in which the usual relationship between latency and sound pressure level is reversed. Since this effect was also found in neurons in the DNLI in the bat (Covey, 1993), this result is useful in establishing that this mechanism is not unique to the echolocating bat. Future research could determine the proportion and distribution of such neurons in DNLL, or study whether such an effect correlates with temporal response pattern, type of tuning curve or other response properties (such as nor-monotonic rate level functions). Inhibitory effects at high stirulus levels may be one possible source of paradoxical latency shift, and it is of interest to determine if paradoxical latency shift correlates with the presence of other manifestations of inhibition (such as closed tuning curves) (Covey, 1993).

\section{Areas Dorsal to DNLL:}

The area dorsal to the main subdivision of DNLL, but ventral to the inferior colliculus, showed a mixture of binaural response properties, including EO/I, EO/F and EO/I mixed responses. While this area may or may not be part of the main division of DNLL, it has been suggested 
(Covey, 1993) that the dorsal part of DNLL in the cat (Brugge et al.. 1970 ) may be analogous to the rostral (monaurally sensitive) division in the bat, since both showed onset response patterns. However, since in the present study, the rostral part of DNLL was found to have high proportions of monaural cells, it could also be possible that the rostral divisions in the rat and bat are analogous. Whether the dorsal region in the DNLL of the rat corresponds to dorsal regions in other species remains unknown.

\section{Rostral DNLL:}

Although sample sizes were small, the binaurai response types (EO/I, EO/O, EO/F) found in rostral DNLL are also comon response types found in the deep layers of the superior colliculus (SCD). The SCD are a presumed projection site for rostral DNLI neurons, and have a topographica? organization with EO/F neurons found in rostral-caudal levels intermediate between between levels for $0 O / F$ and EO/I neurons. Iidi ijoyidpiny iefiects a topography in inaural interaction, fro: sasilitation, through mixed facilitation/inhibition, to increasingly stronger inhibition (Wise and Irvine, 1985; 1983). The SCD have been shown to receive topographically organized input, so it has been suggested that there might also be a similar organization in its inputs (wise and Irvine, 1985). The finding of similar cell types in rostral LNLL, and the finding of EO/I neurons more caudally, followed by EO/O and EO/F responses more rostrally, are results which are compatible with this idea. Furthermore, the neuronal responses measured from the area dorsal to the main subdivision of DNLL showed EO/F and EO/I mixed responses with IID functions similar to those found in SCD; the 
possibility that this dorsal region projects to SCD should be considered.

The frequency tuning found in rostral DNLL neurons in the present study may be similar to the tuning found in some SCD neurons. All SCD neurons respond to broad-band noise, and some are unresponsive to pure tones; however, the units responsive to pure tones can have either Vshaped or broad tuning functions. The sharply tuned neurons are predominantly high-frequency neurons (wise and Irvine, 1983). In the present study, the sample of rostral neurons included high frequency neurons, which had sharp frequency tuning, at least for low sound Fressure levels.

\section{INLL:}

Responses located within the INLL of the rat showed a predominance of monaural (EO/O) sensitivity, but with a significant proportion of binaural (EO/I) sensitivity, which is in accord with earlier stucies showng a partially binaural character of this nucleus (A) $[x$, h ares Brugge, is70; Covey and Casseday, 1986; 1991i. Profortions of. binaural response types found in the present study $\left(63 \% \mathrm{EO} / \mathrm{C} ; 34^{\circ}\right.$ EO/I) are very similar to those found by Aitkin and Brugge (1970) (62\% E0,0: 36 EO'I!. The distribution of binaural responses within INLL found in the present study, with monaural neurons found centrally within INLL, while binaural neurons were found medial to INLL, or close to the dorsal, medial or ventral borders of INLL, has a striking resemblance to the distribution found in the echolocating bat, Eptesicus fuscus (Covey and Casseday, 1991). This suggests that the INLL in the echolocating bat might have a similar organization to that in the rat, 
despite other striking differences in the lemiscal nuclei in the rat and the bat. Unfortunately, it is not possible to draw such comparisons with the data from the cat, since Aitkin and Brugge (1970) did not report any description of the distribution of binaural sensitivity in INLI.

The finding of significant proportions of EO/I neurons in INLI which showed incomplete suppression of firing with ipsilateral stimulation, shows that there are differences in the binaural sensitivity of DNLL and INLL neurons.

The sample of neurons measured from INLL were mainly located in the medial parts of this nucleus, making it difficult to make any observations on the frequency organization in INLL, although the responses which were obtained are at least roughly consistent with the distribution of characteristic frequencies in INLL found in the bat (Covey and Casseday, 1986: 1991). In the bat, highest frequencies were iocated in a small dorsomedial region, high frequencies were found in a LEFgt: zuirolinding medial regjon, and lowest frequencies vere lsated mofe latexally. The data from the present study, in combination with those from earlier studies in the bat (Covey and Casseday, 1991), suggest that the frequency progressions in. INLI may not lie in the dorsal-vertral direction.

\section{Areas Medial to the Lemniscal Nuclei (Paralemniscal Zone):}

Responses were also obtained from regions adjacent to the lemniscal tract which do not form part of the conventionally designated nuclei of the lateral lemniscus (cases BU45, BU46-L), and are part of the lateral tegmentum. These regions medial to DNLL and INLL were found to show 
binaural sensitivity and latencies comparable to those for DNLL, as well as some temporal response patterns similar to those found in DNLL, INLL and VNLL. No obvious frequency organization was found in these regions. Regions medial to DNLL and INLL, and dorsal to DNLL, might correspond to binaurally sensitive regions (paralemniscal zone) found in the bat (Covey and Casseday, 1991; Covey, 1993), although the lack of anatomical information on this region makes this characterization uncertain. In the bat (Covey, 1993), the region medial to the lemniscal nuclei (paralemniscal zone) gave a predominance of binaurally sensitive neurons $(B B E)$, with the largest proportion of these exhibiting EO/I suppression responses, and with a significant proportion of EE sumation responses (248). Binaural facilitation occurred in 128 of the binaural units; the response of these units would be greatly enhanced when sounds were presented binaurally over a certain range of interaural intensity differences.

Anocher recent study, in the horseshoe bat (Metzner, 1993), has cirfodec tha" a function of the paralemniscal area is to control the rlequency of the bat's echolocation calls, by monitoring the characteristiss of the returning echo, and compensating for the flightinduced Dopplex shifts in the echo by lowering the frequency of subsequent vocalization calls. Single unit activity in the paralemiscal zone was monitored during spontaneous vocalizations of bats, and compared with responses to artificially generated auditory stimuli such as playback vocalizations. It was found that when echolocation occurred in a natural situation, responses of paralemiscal neurons depended on the time delay between the orset of vocalization, and simulated echo. The response patterns associated 
with vocalization differed from those triggered by auditory stimuli. even when the auditory stimuli were acoustically identical (Metzner, 1993).

Latencies measured from the paralemniscal zone were comparable to those measured in DNLL, and longer than those found in INLI and VNLL in the bat (Covey, 1993). Thus both the present study, and the studies in the bat (Covey, 1993: Metzner, 1993) found that the lateral tegmentum showed binaural sensitivity and short latencies, which suggest that this area might be part of the ascending binaural auditory system.

The longer latencies in DNLL and the paralemniscal zone, compared with those in the monaural lemniscal nuclei, are consistent with the possibility of there being one more synapse in the pathway by which they receive input from the cochlear nucleus (Covey, 1993). The wide range of latencies exhibited by the DNLI and paralemniscal zone in both the present and previous (Covey, 1993) studies is consistent with the possibility of a diverse set of inputs to these strictures, whicti has a: ready becs confirmed in the case of UNLL; direct foojnotsors from the cochlear nucieus to the DNLL may account for very short latencies. Investigators using a variety of anatomical techniques have given some support for the idea that some ascending auditory afferents terminate in the lateral tegmentum just medial to the lemriscal nuclei (von Monakow, 1890; Held, 1893; Cajal, 1909; Rasmussen, 1960; van Noort, 1969; Elverland, 1978; Glendenning et al., 1981). Morest (1965; proposed that a secondary ascending auditory pathway passes through the tegmental region fom hindbrain to forebrain, based on his finding that lesions in the lateral tegmentum result in degeneration is auditcry nuclei of the thalamus. A more recent study (Glendenring et al., 1981) 
found ascending auditory projections to the lateral tegmentum from the sOC and CN using both anterograde (tritiated leucine transport, and as well as anterograde degeneration techniques) and retrograde tracing (horseradish peroxidase, HRP) techniques. Both anterograde tracing techniques revealed the presence of pericellular terminations on cells in the lateral tegmental region which originated from ips ilateral superior olivary complex, contralateral LSO, and contralateral cochlear nucleus; the largest source of projections appeared to be from the SOC. However, HRP injections which included the lateral tegmentim resulted in almost equal numbers of labeled cells in the contralateral VCN and ipsilateral soc, and it was suggested that limitations in the techniques may have resulted in misleading results for either the anterograde or retrograde tracing daca. In the same study (Glendenning et al., 1981), 2-deoxyyglucose uptake into the lateral tegmentum was found not to increase following pulsating white noise stimulation (continuous for 45 minutes); this was a surprising result since such auditory stimulation results in marked 2 -deoxyglucose uptake ir. other brainstem auditory nuclei. However, the finding of Metzner (1993) that the timing of successive stimuli had an effect on responses, and the finding of habituation effects in the paralemniscal zone in the present study, suggests the possibility that pulsating white noise stimulation may not result in an increased response in the paralemniscal area. One study (Goldberg and Moore, 1967) was unique in finding that the lateral tegmentum medial to the DNLL had commissural projections to contral: cral lateral tegmentum. Knife cut lesions sectioning the paralemniscal zone resulted in fiber degeneration extending across the midline; thus, both the lateral tegmentum and DISLL may have commissural 
projections across the midline (Goldberg and Moore, 1967). However, this result has yet to be replicated with sensitive tracing techniques. It has been suggested (Covey, 1993) that DNLL and the lateral tegmentum are similar in that they both give rise to two very different sets of divergent projections, which include a set of projections to the inferior colliculus, and a set of projections to pre-motor or motor areas. Like DNLL, the lateral tegmentum projects to the central nucleus of the inferior colliculus (Covey and Casseday. 1986): additionally, the lateral tegmentum has reciprocal connections with the deep layers of the superior colliculus (mustache bat: Covey et al.. 1987; cat: Henkel and Edwards, 1978). In the cat, the lateral tegmentum projects to the subdivisions of the facial motor nucleus that innervate the pinna muscles (Henkel and Edwards, 1978). In the awake horseshoe bat, sound-evoked responses of lateral tegmentum neurons are affected by prior vocalization, suggesting a link with pathways that mediate scund production (Covey, 1993). Thus both DNLL and the lateral $l=$ yatialim loz paralemnsscal zonel may provide input to the ascenciang thalamocortical pathway via the ICC, and, in additien, provide a line ketween the binaural auditory pathways and motor pathways for orienting mcrements of the head, eyes, or pinnae (Covey, 1993). The present study, along with the recent study in the echolocating bat (Covey, 1993) thus provide some important new evidence that regions medial to the lemniscal nuclei might be binaurally sensitive, and have some importance in the ascending auditory system. Little information is available on the functional importance of these regiors: thus, these regions present opportunities for future anatomical and physiological study. 
VNLL:

The binaurally sensitive region which was recorded from in VNLL (case BU45) might correspond to the dorsomedial region described in anatomical studies, which receives a dense projection from ipsilateral VCN lcat: Glendenning et al., 1981; Warr, 1969; rhesus monkey:

strominger and strominger, 1971), and on this basis might be expected to be binaurally sensitive. This region could also correspond to a dorsal region of VNLL in the cat (Aitkin et al., 1970) or a medial region in the bat (Covey and Casseday, 1991): in physiological studies, binauraliy sensitive responses (EO/I) in dorsal and ventral areas of Viil were found not to be mingled with monaurally sensitive responses (Aitkin et al., 1970; Covey and Casseday, 1991). However, the study of VWLL neurons in the cat (Guinan et al., 1972ab) did not find such segregation of responses in VNLL. The present. study thus provides som: data suggesting that the projection from ipsilateral cochlear nucdeus

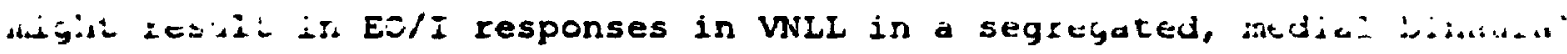
region.

Sagulum:

The sagulum, which has not been recorded from in previous published studies, was distinguished by onset response patterns and long latencies te first response. Although the sample size is small. the data suggest that sagulum is not part of the classic lemniscal ascending auditory pathway. since latencies are longer than what would be expected on the basis of an ascending system. However, sagulum responses showed binaural sensitivity similar to that shown by LNLI. 
neurons, raising possibilities of this region having some importance in sound localization or other binaurally-dependent functions.

Although the nucleus sagulum has not been extensively studied, a recent anatomical study has reported that the sagulum receives efferent fibexs originating from the ipsilateral external and dorsal cortex of the inferior colliculus in the rat (Caicedo and Herbert, 1993), while previous studies in the rat (Faye-Lund, 1986) and monkey (Moore and Goldberg, 1965) found descending projections only from the external cortex. It has been suggested (Caicedo and Herbert, 1993) that this descending collicular pathway could mediate information from the auditory cortex to the sagulum, since both the external and dorsal cortex are major recipients of auditory coitical projections (FayeLund, 1985; Herbert et al., 1991). In the rat and cat, ascending projections from sagulum are to the ipsilateral dorsal and external cortex of IC (Preuß, 1991; Henkel and Shneiderman. 1988), and thus the saguiur. appears to be reciprocally connected with two IC subdivisions.

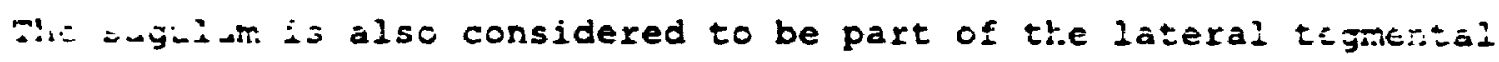
system (Morest, 1965: Morest and Oliver, 1984; Henkel and Shneiderman, 1988), and is the origin of a direct projection (bypassing the inferior colliculus) to the medial and dorsal nucleus of the medial geniculate Lody (Oliver and Hall, 1978a) which provides a parallel ascending pathway to the thalamus. Sirce the sagulum does not appear to receive ary ascending inputs other than those from the inferior colliculus, the collicular pathway to the sagulum presumably provides an auditcry input to this component of the lateral tegmental system (Caicedo and Herbert, 1993). The lcng latencies to the responses in sagulum found in the present study are compatible with apparent lack of ascending input to 
sagulum other than descending inputs from sources with relatively long latencies (such as dorsal and external cortex in IC).

\section{Summary and Implications for Future Research:}

\section{DNLL:}

The present study has found that the DNLL is a binaurally sensitive nucleus; results are consistent with the DNLL playing an important roie in binaural processing in the auditory system. It is unclear from the present study, or previous physiological studies, whether the DNLL has a tonotopic organization; physiological studies up to this point have not been in agreement with anatomical studies on this issue. Future research may employ a combination of physiological and anatomical techniques in order to settle this controversy. Future research could also determine whether different subdivisions are present within DNLL (such as the areas of rostral DNLL, caudal DNLL, or areas dorsal to the main subisivision of DNLI distinguished in the present studyi; the

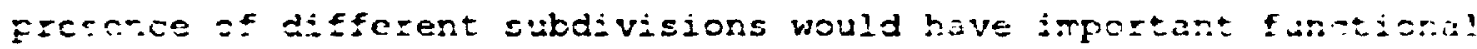
consequeaces and would impact upon the problem of determining tonotck organization.

Other Lemniscal Nuclei:

The monaural or partially binaural character of INLL and VilL suggests a different role for these nuclei in comparison to the DHLL, and future research could undertake to determine the consestivity and functional significance of neurons in the segregated monaural and binaural regions of these nuclei. Other Lemriscal Areas:

Responses were also obtained from sagulum and the paralemniscal 
zone, regions not expected from previous research to be sensitive to auditory stimulation. Thus future research may study the connectivity and cytoarchitecture of these regions within the auditory system, and may further characterize the physiological responses in these regions. 


\section{References:}

Adams, J.C. (1979). Ascending projections to the inferior colliculus. J. Comp. Neurol. 183: 519-538.

Adams, J.C. (1983). Cytology of periolivary cells and the organization of their projections in the cat. J. Comp. Neurol. 215: 275-289.

Adams, J.C. and Mugnaini. E. (1984). Dorsal nucleus of the lateral lemniscus: a nucleus of GABAergic projection neurons. Brain Res. Bull. 13: 585-590.

Adams, J.C. and Mugnaini, E. (1990). Immunocytochemical evidence for inhibitory and disinhibitory circuits in the superior olive. Hear.

Res. 49: 281-298.

Adams, J.C. and Warr, W.B. (1976). Origins of axons in the cat's acoustic strias determined by injection of horseradish peroxidase into severed tracts. J. Comp. Neurol. 170: 107-122.

Adams, J.C. and Wenthold, R.J. (1987). Immunosta..ning of ascending auditory pathways with glycine antiserum. Absts. Midwinter Mtg. Assoc. Res. Otolaryngol. 1987 10: 63.

Aitkin, L.M. and Phililips, S.C. (1984). The interconnections of the inferior colliculi through their commissure. J. Comp. Neurol. 228: 210-216.

Aitkin, I.M. and Schuck, D.M. (1985). Low frequency neurons in the lateral central nucleus of the cat inferior colliculus receive their input predominantly from the medial superior olive. Hear. Res. 17: $87-93$.

Aitkin, L.M., Anderson, D.J. and Brugge, J.F. (1970). Tonotopic organization and discharge characteristics of single neurons in nuclei oi the lateral lemniscus of the cat. J. Neurophysiol. 33: 421-440.

Aitkin, L.M. et al. (1963). Inferior colliculus. I. Comparison of response properties of neurons in the central, pericentral and external nuclei of adult cat. J. Neurophysiol. 38: 1196-1207.

Aitkin, L.M., Webster, W.R., Veale, J.L. and Crosby, D.C. (1975). Inferior colliculus. I. Comparison of response properties of neurons in the central, pericentral, and external nuclei of adult cat. J. Neurophysiol. 38: 1196-1207.

Allon, N. and Wollberg, Z. (1978). Responses of cells in the superior collzculus of the squirrel monkey to auditory stimuli. Brain Res. 159: $321-330$.

Altshiler, R.A. et al. (1986). Identification of glycinergic synapses in the cochlear nucleus through immunocytochemical localization of the postsynaptic receptor. Brain Res. 369: 316-320.

Andersen, R.A., Synder, R.L. and Merzenich, M.M. (1980). The topographic organization of corticocollicular projections from physiologically identified loci in the AI, AII, and anterior cortical fields of the cat. J. Comp. Neurol. 191: 479-494.

Aoki, E., Semba, R., Keino, R., Kato, K. and Kashiwamata, S. (1988). Glycine-1ike immunoreactivity in the rat auditory pathway. Brain Res. 442: 63-71.

Bajo, V.M.. Merchan, M.A., Lopez, D.E. and Rouiller, E.M. (1993). Netironal Morphology and Efferent Projections of the Dorsal Nucleus of the Lateral Lemniscus in the Rat. J. Comp. Neurol. 334: 241-262.

Beyerl, B.D. (1978). Afferent projections to the central nucleus of the inferior colliculus in the rat. Brain Res. 145: 209-223.

Bledsce, S.C., Snead, '.R., Helfert, R.H., Prasad, V.. Wenthold, R.J. and Altschuler, R. .. (1990). Immunocytochemical and lesion studies support the hypotnesis that the projection from the medial nucleus of the trapezoid body to the lateral superior olive is glycinergic. Brain Res. 517: 189-194.

Borg, E. (1973a). A neuroanatomical study of the brainstem auditory 
system of the rabbit. Part I. Ascending connections. Acta Morphol. Neerl. Scand. 11 : $31-48$.

Borg, E. $(1973 \mathrm{~b})$. On the neuronal organization of the acoustic middle ear reflex. A physiological and anatomical study. Brain Res. 49: 101-123.

Boudreau, J.C. and Tsuchitani, C. (1968). Binaural interaction in the cat superior olive s segment. J. Neurophysiol. 31: 442-454.

Boudreau, J.C. and Tsuchitani, C. (1970). Cat superior olive s-segment cell discharge to tonal stimulation. In: Neff, W.D.. ed.

Contributions to sensory physiology, vol. 4. New York: Academic, pp. 143-213.

Bourk, T.R. (1976). Electrical responses of neural units in the anteroventral cochlear nucleus of the cat. Ph.D. Thesis, Massachusetts Institute of Technology. Cambridge, MA.

Brawer, J.R., Morest, D.K. and Kane, E.C. (1974). The neuronal architecture of the cochlear nucleus of the cat. J. Comp. Neurol. 155: 251-299.

Browne11. W.E. (1975). Organization of the cat trapezoid body and the discharge characteristics of its fibers. Brain Res. 94: 413-433.

Brownell, W.E.. Manis, P.B., Ritz, L.A. (1979). Ipsilateral inhibitory responses in the cat lateral superior olive. Brain Res. 177: 189193.

Browner, R.H. and Webster, D.B. (1975). Projections of the trapezoid body and the superior olivary complex of the kangaroo rat (Dipodomys merriami). Brain Behav. Evol. 11: 322-354.

Brugge, J.F., Anderson, D.J. and Aitkin, L.M. (1970). Responses of neurons in the dorsal nucleus of the lateral lemniscus of the cat to binaural tonal stimulation. J. Neurophysiol. 33: 44I-458.

Brunso-Bechtold, J.K., Thompson, G.C. and Masterton, R.B. (1981). HRP study of the organization of auditory afferents ascending to the central nucleus of the inferior colliculus in cat. $J$. Comp. Neurol. 197: 705-722.

Caicedo, A. and Herbert, H. (1993). Topography of descending projections from the inferior colliculus to auditory brainstem rucies in the rat. J. Comp. Neurol. 328: 377-392.

Caird, $\dot{\nu}$ and Klinke, R. (1983). Processing of binaural stimuli by cat superior olivary complex neurons. Exp. Brain Res. 52: 385-399.

cajal. Ramon y S. (1909). Histologle du system nerveux de l'home et des vertebres. Tome, I: Instituto Ramon y Cajal, Madric, 1952, Pp. $774-838$.

Cant, N.B. (1984). The fire structure of the lateral superior olivary nucleus of the cat. J. Comp. Neurol. 227: 63-77.

Cant. N.B. and Casseday, J.H. (1986). Projections from anteroventral cochlear nucleus to the lateral and medial superior olivary nuclei. J. Comp. Neurol. 247: 447-476.

Cant, N.B. and Morest, D.K. (1979a). Organization of the reurons in the anterior division of the anteroventral cochlear nucleus of the cat. Light-microscopic observations. Neuroscience 4: 1909-1923.

Cant, N.B. and Morest, D.K. (1979b). The bushy cells in the anteroventral cochlear nucleus of the cat. A study with the electron microscope. Neuroscience 4: 1925-1945.

Cant. N.B. (1991). Projections to the lateral and medial superior olivary nuclei from the spherical and globular bushy cells of the anteroventral cochlear nucleus. In: R.A. Altschuler et al., eds. Neurobiology of hearing. New York: Raven Press.

Caspary, D.H.. Rybak, L.P and Faingold, C.L. (1985). The effects of 1nhibitory and excitatory neurotransmitters on the response properties of brainstem auditory neurons. In: Drescher, D.G., ed. Auditory biochemistry. Springfield, IL: Charles C. Thomas, PP. 198-226. 
Casseday, J.H. and Covey, E. (1987). Central auditory pathways in directional hearing. In: Yost, W.A., Gourvitch, G., eds. Directional Hearing. New York: Springer-Verlag, pp. 109-145.

Caspary, D.M., Havey, D.C. and Faingold, C.L. (1979). Effects of microiontophoretically applied glycine and GABA on neuronal response patterns in the cochlear nuclei. Brain Res. 172: 179-185.

Casseday, J.H. and Neff. W.D. (1975). Auditory Localization: Role of Auditory Pathways in Brain Stem of the Cat. J. Neurophysiol. 38: 842-858.

Chalupa, L.M. and Rhoades, R.W. (1977). Responses of visual, somatosensory, and auditory neurons in the golden hamster's superior colliculus. J. Physiol. (Lond.) 270: 595-626.

Coleman, J.R. and clerici, W.J. (1987). Sources of projections to subdivisions of the inferior colliculus in the rat. J. Comp. Neurol. 262: 215-226.

Conlee, J.w. (1979). Descending auditory projections from the inferior colliculus and nuclei of the lateral lemniscus in the cat. Ph.D. dissertation, Chicago, University of Chicago.

Covey. E. (1993). Response properties of single units in the dorsal nucleus of the lateral lemniscus and paralemniscal zone of an echolocating bat. J. Neurophysiol. 69(3): 643-859.

Covey, E. and Casseday, J.H. (1986). Connectional basis for frequency representation in the nuclei of the lateral lemniscus of the bat Eptesicus fuscus. J. Neurosci. 6: 2926-2940.

Covey. E. and Casseday, J.H. (1991). The monaural nuclei of the lateral lemniscus in an echolocating bat: parallel pathway for analyzing temporal features of sound. $J$. Neurosci. 11: 3456-3470.

Covey, E.. Hall, W.C. and Kobler, J.B. (1987). Subcortical connections of the superior colliculus in the mustache bat. Pteronotus parnellii. J. Comp. Neurol. 263: 179-197.

Diamond, I.T. et al. (1969). The projection of the auditory cortex upon the diencephalon brain stem in the cat. Brain Res. 15: 305-340.

Diamond, I.T. et al. (1973). Neuroanatomy of the auditory system. Arci. Otolaryngol. 9B: 397-412.

Enret. G. and Moffat, A.J.M. (1985). Inferior colliculus of the nouse mouse. II. Single unit responses to tones, noise and tone-noise combinations. J. Comp. Physiol. [A] 156: 619-635.

Eiverlant, H.H. (1978). Ascending and intrinsic projections of t.t supericr olivary complex in the cat. Exp. Brain Res. 32: 117-134.

Evans, E.5. and Nelson, P.G. (1973). The responses of single neurcns in the cochlear nucleus of the cat as a function of their location and anaesthetic state. Exp. Brain Res. 17: 402-427.

Faingold, C.I., Gehlbach, G. and Caspary, D.M. (1989). On the role of GRBA as an inhibitory neurotransmitter in inferior colliculus neurons: iontophoretic studies. Brain Res. 500: 302-312.

Eaingsid, C.I., Boersma Anderson, C.A. and Caspary, D.M. 11991i. Involvement of GABA in acoustically-evoked inhibition in inferior colliculus neurons. Hearing Res. 52: 201-216.

Faye-Lund, H. (1985). The neocortical projection to the inftrior coiliculus in the albino rat. Anat. Embryol. 173: 53-70.

Faye-Lund, H. (1986). Projection from the inferior colliculus $=0$ the superior olivary complex in the albino rat. Anat. Embryol. (Berl.) 175: 35-52.

Fekete, D.M. et al. (1984). The central projections of intracellularly labelled auditory nerve fibers in cats. J. Comp. Neurol. 229: 432450.

Fernaridez, C. and Karapas, F. (1967). The course and termination of the striae of Monakow and Held in the cat. J. Comp. Neurol. 131: 371-386.

Finlayson, P.G. and Caspary, D.M. (1991). Low-frequency neurons in the 
lateral superior olive exhibit phasesensitive binaural inhibition. J. Neurophysiol. 65: 598-604.

EitzPatrick, K.A. and Imig, T.J. (1978). Projections of auditory cortex uopn the thalamus and midbrain in the owl monkey. J. Comp. Neurol. 177: 537-556.

Friauf, E. and Ostwald, J. (1988). Divergent projections of physiologically characterized rat ventral cochlear nucleus neurons as shown by intra-axonal injection of horseradish peroxidase. Exp. Brain Res. 73: 263-284.

Frostholm, A. and Rotter, A. (1986). Autoradiographic localization of receptors in the cochlear nucleus of the mouse. Brain Res. Bull. 16: 189-203.

Glendenning, K.K., Brunso-Bechtold, J.K., Thompson, G.C. and Masterton, R.B. (1981). Ascending auditory afferents to the nuclei of the lateral lemniscus. J. Comp. Neurol. 197: 673-703.

Glendenning, K.K.. Hutson, K.A., Nudo, R.J., and Masterton, R.B. (1985). Acoustic chiasm II: anatomical basis of binaurality in lateral superior olive of cat. J. Comp. Neurol. 232: 261-285.

Glendenning, K.K. and Baker, B.N. (198B). Neuroanatomical distribution of receptors for three potential inhibitory neurotransmitters in the brainstem auditory nuclei of the cat. J. Comp. Neurol. 275: 288308 .

Glendenning, K.K. and Masterton, R.B. (1983). Acoustic chiasm: efferent projections of the lateral superior olive. J. Neurosci. 3: $1521-1537$.

Godf́rey, D.A.. Kiang, N.Y.S., and Norris, B.E. (1975a). Single unit activity in the posteroventral cochlear nucleus of the cat. $J$. Comp. Neurol. 162: 247-268.

Godfrey, D.A., Kiang, N.Y.S., and Norris, B.E. (1975b). Single unit activity in the dorsal cochlear nucleus of the cat. J. Comp. Neurol. 162: 269-284.

Godfrey, D.A. et al. (1977). Quantitative histochemical mapping of candidate transmitter amino acids in the cat cochlear nucleus. J. Histochem. Cytochem. 25: 417-431.

sodfrey, D.A. et al. (19B8). Neurotransmitter neuromicroshemistry of the cochlear nucleus and superior olivary complex. In: Syka, J.. Masterton, R.D., eds. Auditory pathway. New York: Plenum, 1988, PF. 107-121.

Goldberg. J.M. and Brown, P.B. (1968). Functional organization of the doy superior olivary complex: an anatomical and electrophysiological study. J. Neurophysiol. 31: 639-656.

Goldberg. J.M. and Brown, P.B. (1969). Response of binaural neurons of dog superior olivary complex to dichotic tonal stimuli: some physiological mechanisms of sound localization. J. Neurophysiol. 32:613-636.

Guldberg, J.M. and Brownell, W.E. (1973). Discharge characteristics of neurons in anteroventral and dorsal cochlear nuclei of cat. Brain Res. 64: 35-54.

Goidberg, J.M. and Moore, R.Y. (1967). Ascending projections of the lateral iemniscus in the cat and monkey. J. Comp. Neurol. 129: 143156.

Goldberg, J.M., Smith, F.D. and Adrian, H.O. (1963). Response of single units of superior olivary complex of the cat to acoustic stimuli: laterality of afferent projections. Anat. Rec. 145: 232 .

Gonzalez-Hernandez, T.H. et al. (1987). Afferent connections of the inferior colliculus in the albino mouse. $J$. Hirnforsch 3 : 315-323.

Greenamyre, J.T., Young, A.B., and Penney, J.B. (1984). Quantitative autoradiographic distribution of $\mathrm{L}-(3 \mathrm{H})$ glutamate-binding sites in rat central nervous system. J. Neurosci. 4: 2133-2144.

Guinan, J.J. Jr., Guinan, S.S. and Norris, B.E. (1972a). Single 
auditory units in the superior olivary complex. I. Responses to sounds and classifications based on physiological properties. Int. J. Neurosci. 4: 101-120.

Guinan, J.J. Jr.. Norris, B.E. and Guinan, S.S. (1972b). Single auditory units in the superior olivary complex. II: Locations of unit categories and tonotopic organization. Int. J. Neurosci. I: 147-166.

Halpain, S., Wieczorek, C.M. and Rainbow, T.C. (1984). Localization of I-glutamate receptors in rat brain by quantitative autoradiography. J. Neurosci. 4: 2247-2258.

Harnischfeger, G., Neuweiler, G. and Schlegel, P. (1985). Interaural time and intensity coding in superior olivary complex and inferior colliculus of the echolocating bat Molossus ater. J. Neurophysiol. 53: 89-109.

Harrison, J.M. and Feldman, M.L. (1970). Anatomical aspects of the cochlear nucleus and superior olivary complex. In: Neff, W.D. (ed.) Contributions to sensory physiology, vol. 4. Academic, New York, pp. 95-142.

Harrison, J.M. and Howe, M.E. (1974). Anatomy of the afferent auditory nervous system of marmals. In: Keidel, W.D., Neff, W.D. (eds.) Handbook of sensory physiology, vol. V. Auditory system, part 1. Springer, New York, PP. 284-336.

Harrison, J.M. and Irving, R. (1966a). Visual and nonvisual auditory systems in mammals. Science 154: 738-743.

Harrison, J.M. and Irvine, R. $(1 \overline{966} \mathrm{~b})$. Ascending connections of the anterior ventral cochlear nucleus in $t^{2} . e$ rat. J. Comp. Neurol. 126: $51-64$.

Harrison, J.M. and warI, W.B. (1962). The cochlear nucleus and ascending pathways of the medulla. J. Comp. Neurol. 119: 341-380.

Havey, D.C. and Caspary, D.M. (1980). A simple technique for constructing 'piggy-back' multibarrel microelectrodes. Electroencephal. Clin. Neurophysiol. 48: 249-251.

Held, H. (1893). Die Zentraie Gehorleitung. Arch. Anat. Physiol., Anat. Alt. 17: 201-248.

Helferc, R.H. and Schwartz, I.R. (1986). Morphological evidence for the existence of multiple neuronal classes in the cat lateral superior olivary nucleus. J. Comp. Neurol. 244: 533-549.

Helfert, R.H. and Schwartz, I.R. (1987). Morphological features of five neuronal classes in the gerbil lateral superior olive. Am. J. Anat. 179: 55-69.

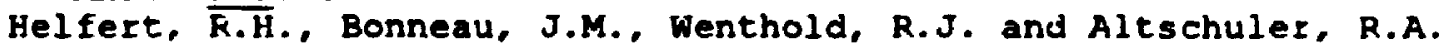
(1989). GABA and glycine immunoreactivity in the guined pig sipericr olivary complex. Brain Res. 501: 269-286.

Helfert, R.H., Juiz, J.M., Bledsoe, S.C., Jr., Bonneau, J.M., Wenthold, R.J. and Altschuler, R.A. (1992). Patterns of glutamate, glycine and GABA immunolabeling in four synaptic terminal classes in the lateral superior olive of the guinea pig. J. Comp. Neurol.. in press.

Helfert, R.H. et al. (1991). The ascending auditory pathways. In: Neurobiology of hearing: The central auditory system. Altschuler, R.A. et al., eds. New York: Raven Press, pp. 1-25.

Henkel, C.K. and Edwards, S.B. (1978). The superior colliculus contral of pinna movements in the cat: possible anatomical connections. $J$. Comp. Neurol. 182: 763-776.

Henkel, C.K. and Shneiderman, A. (1988). Nucleus sagulum: Projections of a lateral tegmental area to the inferior colliculus in the cat. J. Comp. Neurol. 271: 577-588.

Henkel, C.K. and Spangler, K.M. (1983). Organization of the efferent projections of the medial superior olivary nucleus in the cat as revealed by HRP and autoradiographic tracing methods. J. Comp. 
Neurol. 221: 416-420.

Herbert, H.. Aschoff, A. and Ostwald, J. (1991). Topography of projections from the audicory cortex to the inferior colliculus in the rat. J. Comp. Neurol. 304: 1-20.

Hironaka, T.. Morita, Y., Hagihira, S., Tateno, E.. Kita, H. and Tohyama, M. (1990). Localization of GABA-A receptor al subunit mRNA-containing neurons in the lower brainstem of the rat. Molecular Brain Res. 7: 335-345.

Hutson, K.A. $(\overline{1} 988)$. Connectons of the auditory midbrain: efferent projectons of the dorsal nucleus of the lateral lemniscus, the nucleus sagulum, and the origins of the GABAergic commissure of Probst. Doctoral dissertation. Florida State University. Tallahassee, FL.

Hutson, K.A., Glendenning, K.K. and Masterton, R.B. (1987). Biochemical basis for the acoustic chiasm? Soc. Neurosci. Abstr. 13: 548 .

Hutson, K.A., Glendenning, K.K. and Masterton, R.B. (1991). Acoustic chiasm IV: Eight midbrain decussations of the auditory system in the cat. J. Comp. Neurol. 312: 105-131.

Inbody, S.B. and Feng, A.S. (1981). Binaural response sharacteristics of single neurons in the medial superior olivary nucleus of the albino rat. Brain Res. 210: 361-366.

Irvine, D.R.F. (ed.) (1986). Progress in Sensory Physiology Vol.?. Springer-Verlag, Berlin. pp.125, 78, 96-99.

Irving, R. and Harrison, J.M. (1967). The superior olivary complex and audition: a comparative study. J. Comp. Neurol. 130: 77-86.

Iwahori. N. (1986). A Golgi study on the dorsal nucleus of the lateral lemniscus in the mouse. Neurosci. Res. 3: 196-212.

Jean-Baptiste, M. and Morest, D.K. (1975). Transneuronal changes of synaptic endings and nuclear chromatin in the trapezoid body following cochlear ablations in cats. J. Comp. Neurol. 162: 111-134.

Jenkins. W.M. and Masterton, R.B. (1982). Sound localization: effects of unilaterai lesions in central auditory system. J. Neurophysiol. 47: $987-1016$.

Johrisor. J.W. and Ascher, P. (1987). Glycine potertiates the Mari response in cultured mouse brain neurons. Nature 325: 529-53:.

Juiz, J.M. et al. (1989). Immunocytochemical localization of the Gitas/benzodiazepine receptor in the gusnea pig cochieav ruiteis: evidence for receptor localization heterogeneity. Erair. Res. 504: $173-179$.

Kane, E.C. (1974). Patterns of degeneration in the caudal ccchlear nucleus of the cat after cochlear ablation. Anat. Rec. 179: 67-91.

Kavanagh, G.L. and Kelly, J.B. (1988). Hearing in the ferret (Mustela putorius): effects of primary auditory cortical lesions on thresholds for pure tone detection. J. Neurophysiol. 60: 879-5é.

Kavanagh, G.L. and Kelly, J.B. (1992). Midline and lateril field sound localization in the ferret (Mustela putorlus): contribution of the superior olivary complex. J. Neurophysioi. 67: 1643-1658.

Kelly. J.B., Judge, P.W. and Phillips, D.P. (1986). Representation of the cochlea in primary auditory cortex of the ferret Mustela putorius). Hearing Res. 24: 111-115.

Kelly, J.B., Kavanagh, G.L. and Dalton, J.C.H. (1986). Hearing in the ferret (Mustela putorius): thresholds for pure tone detection. Hearing Res. 24: 269-275.

Kelly, J.B., Kavanagh, G.L. and Picton, T.W. (1989). Brainstem auditory evoked response in the ferret (Mustela putozius). Hearing Res. 39: 231-240.

Kelly, J.B. and Sally, S.L. (1988). Organization of auditory cortex in the albino rat: binaural response properties. J. Neurophysiol. 59: 1756-1769. 
Kudo, M. (1981). Projections of the nuclei of the lateral lemniscus in the cat: an autoradiographic study. Brain Res. 221: 57-69.

Kuwada, S. and Yin, T.C.T. (1983). Binaural interaction in low frequency neurons in the inferior colliculus of the cat. I. Effects of long interaural delays, intensity, and repetition rate on interaural delay function. J. Neurophysiol. 50: 981-999.

Kuwabara, N.. DiCaprio, R.A. and zook, J.M. (199\%). Afferents to the medial nucleus of the trapezoid body and their collateral projections. J. Comp. Neurol. 314: 684-706.

Li, R.Y.S. and Guinan, J.J. (1971). Antidromic and orthodromic stimulation of neurons receiving calyces of Held. Quarterly progress report, research laboratory of electronics. MIT 100: 227234.

Li, L. and Kelly, J.B. (1991). A reversible block of neural activity in DNLI alter binaural responses in contralateral inferior colliculus. Assoc. Res. Otolaryngology Abstr. 15: 62 .

Li, L. and Kelly, J.B. (1992). Binaural responses in rat inferior colliculus following kainic acid lesions of the superior olive: interaural intensity difference functions. Hearing Res. 61: 73-85.

Maffi, C.I. and Aitkin, L.M. (1987). Difierential neural projections to regions of the inferior colliculus of the cat responsive to high frequency sounds, Hear. Res. 26: 211-219.

Mast, T.E. and Chung, D.Y. (1973). Binaural interaction in the superior colliculus of the chinchilla. Brain Res. 42: 227-230.

Masterton, R.B. (1974). Adaptation for sound localization in the ear and brainstem of mammals. Fed. Proc. 33: 1904-1910.

Masterton, R.B. and Diamond, I.T. (1967). Medial superior olive and sound localization. Science 155: 1696-1697.

Masterton, R.B., Jane, J.A. and Diamond, I.T. (1967). Role of brainstem auditory structures in sound localization. I. Trapezoid body, superior olive, and lateral lemniscus. J. Neurophysiol. 30: 341359.

Masterton, R.B. et al. (1975). Neuroanatomical basis of binaural phase-difference analysis for sound localization: a comparative study. J. Comp. Physiol. Psychol. 89: 379-386.

Masterton, R.B. et al. (1979). Preservation of trapezoid body fibers after neurochemical ablation of the superior olives with kainic acid. Brain Res. 173: 156-159.

Merzenich, M.M. and Reid, M.D. (1974). Representation of the cochlea within the inferior colliculus of the cat. Brain Res. 77: 397-415.

Metzner, w. (1989). A possible neuronal basis for Doppler-shift compensation in echo-locating horseshoe bats. Nature (Lond.) 341 : $529-532$.

Metzner, $w$. (1993). An audio vocal interface in echolocating horseshoe bats. J. Neuroscience 13(5): 1899-1915.

Metzner, W. and Radtke-Schuller, S. (1987). The nuclei of the lateral lemniscus in the horseshoe bat, Rhinolophus rouxi. J. Comp.

Physiol. A 160: 395-411.

Mclaughlin, B.J., Wood, J.G., Saito, K., Roberts, E. and Wu, J.-Y. (1974). The fine structural localization of glutamate decarboxylase in synaptic terminals of rodent cerebelium. Brain Res. 76: 377-391.

Monaghan, D.T. and Cotman, C.W. (1985). Distribution of N-methyl-Daspartate-sensitive $\mathrm{I}-3 \mathrm{H}$-glutamate-binding sites in rat brain. J. Neurosci. 5: 2909-2919.

Monakow, C. vón (1890). Striae acusticae und untere Schliefe. Arch. Psychiatx. Nervenkrankh., 22: 1-29.

Moore, J.K. (1987). The human auditory brain stem: a comparative view. Hear. Res. 29: 1-32.

Mocre, M.J. and Caspary, D.M. (1983). Strychnine blocks binaural inhibition in lateral superior olivary neurons. J. Neurosc1. 3: 237- 
242.

Moore, R.Y. and Goldberg, J.M. (1966). Projections of the inferior colliculus in the monkey. Exp. Neurol. 14: 429-438.

Moore, J.K. and Moore, R.Y. (1971). A comparative study of the superior olivary complex in the primate brain. Folia Primatol. (Basel) 16: 35-51.

Moore, J.K. (1980). The primate cochlear nuclei: loss of lamination as a phylogenetic process. J. Comp. Neurol. 193: 609-629.

Moore, J.K. and Moore, R.Y. (1987). Glutamic acid decarboxylase-like immunoreactivity in brainstem auditory nuclei of the rat. J. Comp. Neurol. 260: 157-174.

Moore, D.R.. Semple, M.N. and Addison, P.D. (1983). Some acoustic properties of neurones in the ferret inferior colliculus. Brain Res. 269: 69-82.

Morest, D.K. (1965). The lateral tegmental system of the midbrain and the medial geniculate body: A study ith Golgi and Nauta methods in cat. J. Anat. (Lond.) 99: 611-634.

Morest, D.K. (1968a). The collateral system of the medial nucleus of the trapezoid body of the cat, its neuronal architecture and relation to the olivo-cochlear bundle. Brain Res. 9: 288-311.

Morest, D.K. (1968b). The growth of synaptic endings in mammalian brain: a study of the calyces of the trapezoid body. 2 . Anat. Entwicklungsgesch 127: 201-220.

Morest, D.K. and Oliver, D.L. (1984). The neuronal architecture of the inferior colliculus in the cat: defining the functional anatomy of the auditory midbrain. J. Comp. Neurol. 222: 209-236.

Mugnaini et al. (1980). Distribution and light microscopic features of granule cells in the cochlear nuclei of cat, rat and mouse. $J$. Comp. Neurol. 191: 581-606.

Nordeen, K.w. et al. (1983). Ascending auditory projections to the inferior colliculus in the adult gerbil, Meriones unguiculatus. J. Comp. Neurol. 214: 131-143.

Nudo, R.J. and Masterton, R.B. (1984). 2-Deoxyglucose studies of stimulus coding in the brainstem auditory system of the cat. In: NefE. W.D. (ed.) Contributions to sensory physiology, vol. .. Academic, London, PP. 79-97.

Oliver, D.L. (1984). Dorsal cochlear nucleus projections to the inierior colliculus in the cat: a light ano electzon microscopic study. J. Comp. Neuro1. 224: 155-172.

Cliver. D.I. (1985). Quantitative analyses of axonal endings in the central nucleus of the inferior colliculus and distribution of $3 \mathrm{H}-$ labeling after injections in the dorsal cochlear nucleus. J. Comp. Neurol. 237: 343-359.

oliver, D.I. (1987). Projections to the inferior colliculus from the antercventral cochlear nucleus in the cat: possible substrates for binaliral interaction. J. Comp. Neurol. 264: 24-46.

Oliver, D.L. and Hall, w.C. (1978a). The medial geniculate body of the tree shrew, Tupaia glis. I. Cytoarchitecture and midbrain connections. J. Comp. Neurol. 182: 423-458.

Oliver. D.I. and Hall, w.C. $(1978 b)$. The medial geniculate body of the tree shrew, Tupaia glis. II. Connections with the neocortex. J. Comp. Neurol. 182: 459-494.

Oliver, D.L. and Morest, D.K. (1984). The central nucleus of the inferior colliculus in the cat. J. Comp. Neurol. 222: 237-264.

Oliver, D.L. and Shneiderman, A. (1989). An EM st:- ; of the dorsal nucleus of the lateral lemniscus: inhibitory, commissural, synaptic connections between ascending auditory pathways. J. Nelrosci. 9: 967-982.

Oliver, D., Schneiderman, A., and Henkel, C.K. (1987). Morphological substrates for binaural interactions in the midbrain: the dorsal 
nucleus of the lateral lemniscus. ARo Absts. 10: 218-219.

Osen, K.K. (1969). Cytoarchitecture of the cochlear nuclei in the cat. J. Comp. Neurol. 136: 453-484.

Osen. K.K. (1970). Course and termination of the primary afferents in the cochlear nuclei of the cat. An experimental anatomical study. Arch. Ital. Biol. 108: 21-51.

Osen, K.K. (1972). Projections of the cochlear nuclei on the inferior colliculus in the cat. J. Comp. Neurol. 144: 355-372.

Osen, K.K. et al. (1984). Histochemical localizations of acetylcholinesterase in the cochlear nucleus and superior olivary nuclei. A reappraisal with emphasis on the cochlear granule cell systems. Arch. Ital. Biol. 122: 169-212.

Papez. J.W. (1929). Comparative Neurology. New York: Haffner Publishing Company. pp. 270-293.

Peyret, D. et al. (1987). Glycine immunoreactivity in the brainstem auditory and vestibular nuclei of the guinea pig. Acta otolaryngol. (stockh.) 104: 71-76.

Pfeiffer, R.R. (1966). Classification of response patterns of spike discharges for units in the cochlear nucleus: Tone burst stimulation. Exp. Brain Res. 1: 220-235.

Phillips, D.P., Judge, P.W. and Kelly. J.B. (1988). Primary auditory cortex in the ferret (Mustela putorius): neural response properties and topographic organization. Brain Res. 443: 281-294.

Phillips, D.P. and Kelly, J.B. (1989). Coding of tonepulse amplitude by single neurons in auditory cortex of albino rats (Rattus norvegicus). Hearing Res. 37: 269-280.

Phillips, D.F. and Kelly. J.B. (1992). Effects of continuous noise maskers on tone-evoked potentials in cat primary auditory cortex. Cerebral Cortex 2: 134-140.

Pickles, J.O. (1988). An Introduction to the Physiology of Hearing. Academic Press, San Diego.

Potashner, S.J., Lindberg, N. and Morest, D.K. (1985). Uptake and release of gamma-aminobutyric acid in the guinea pig cochlear nucleus after axotomy of cochlear and centrifugal fibers. J. Ne:rochem. i5: 1558-1566.

Rotáshner, S.J.. Scheiderman, A., ' hase, M.B., Benson, C. and Rockwood, J.M. (1991). GABA and glycine release from guinea pig inferiur

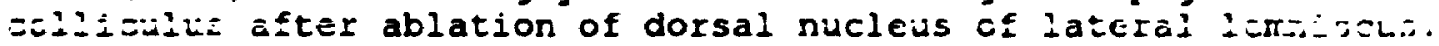
soc. Neurosc1. Abstr. 17: 300 .

Freuß, S. (1991). Elektrophysiologische und neuroanatomische Charakterisierung der Kerne des Lateralen Lemniscus und der umgebenden auditorischen Areale bei der Ratte. Thesis, Universitat Tubingen.

Rasmussen, G.L. (1960). Efferent fibers of the cochlear nerve and cochiear nucleus. In: Neuronal Mechanisms of the Auditory and Vestibular systems. G.L. Rasmussen and W.F. Windle (eds.). Themas, Springfiela, Ill.. Pp. 105-115.

Rasmussen, G.L. (1967). Efferent connections of the cochlear nucleus. Ir: Graham, A.B. (ed.) Sensorineural hearing processes and disorders. Little Brown, Boston, pp. 61-75.

Rhode, V.S., Oertel, D. and Smith, P.H. (1983a). Physiological response properties of cells labelled intracellularly with horseradish peroxidase in cat ventral cochlear nucleus. J. Comp. Neurol. 213: 448-463.

Rhode, W.S., Smith, P.H., and Oertel, D. (1983b). Physzologicii response properties of cells labelled intracellularly with horseradish peroxidase in cat dorsal cochlear nucleus. J. Comp. Neurol. 213: 426-447.

RoBards, M.J. (1979). Somatic neurons in the brain stem and neocortex 
projecting to the external nucleus of the inferior colliculus: anatomical study in the opossum. J. Comp. Neurol. 184: 547-566.

Roberts, R.C. and Ribak, C.E. (1987). GABAergic neurons and axon terminals in the brainstem auditory nuclei of the gerbil. J. Comp. Neurol. 258: 267-280.

Rooney, B.J., Kavanagh, G.L. and Kelly, J.B. (1991). Kainic acid lesions of the superior olivary complex: a horseradish peroxidase study of surviving brainstem projections. J. Neurosci. Meth. 39: 65-75.

Rose, J.E., Greenwood, D.D., Goldberg, J.M, and Hind, J.E. (1963). Some discharge characteristics of si:sgle neurons in the inferior colliculus of the cat. I. Tonotopical organization, relation of spike-counts to tone intensity, and firing patterns of single elements. J. Neurophysiol. 26: 294-320.

Rose. J.E. (1960). Organization of frequency sensitive neurons in the cochlear nuclear complex of the cat. In: Rasmussen, G.L. Windle, W.F. (eds.) Neural mechanisms of the auditory and vestibular systems. Thomas, Springfield, pp. 116-136.

Rose, J.E., Galambos, R.. and Hughes, J.R. (1959). Microelect rode studies of the cochlear nuclei of the cat. Bull. Johns Hopkins Hosp. 104: 211-251.

Rose, J.E. et al. (1963). Some discharge characteristics of single neurons in the inferior colliculus of the cat. I. Tonotopical organisotion, relation of spike counts to tone intensity and firing patterns of single elements. J. Neurophysiol. 26: 294-320.

Rose, J.E. et al. (1966). Some neural mechanisms in the inferior colliculus of the cat which may be relevant to localization of a sound source. J. Neurophysiol. 29: 288-314.

Roth, G.L.. Aitkin, L.M., Anderson, R.A. and Merzenich, M.M. (1978). Some features of the spatial organization of the central nucleus of the inferior nd Ryugo, D. (1984). Intracellular marking of physiologically characterized cells in the ventral cochiear nucleus of the cat. J. Comp. Neurol. 225: 167-186.

Ryir., A.F. et al. (1982). Tonotopic organization in the central auditory pathway of the Mongolian gerbil: a 2-deoxyglucose stucy. J. Comp. Neurol. 207: 369-380.

Ryan, A.F., Woolf, N.K. and Sharp. F.R. (1982). Tonotopic organization 25. true central auditory pathway of the mongolian gerbil: a 2deoxyglucose study. J. Comp. Neurol. 207: 369-380.

Ryuyo, D.K. and Willard, F.H. (1985). The dorsal cochlear nuclecis of the mouse: a light microscopic analysis of neurons that project to the inferior colliculus. J. Comp. Neurol. 242: 381-396.

Ryugo, D.K., Willard, F.H. and Fekete, D.M. (1981). Differential afferent projections to the inferior colliculus from the cochlear ajcleus in the albino mouse. Brain Res. 210: 342-349.

Rjugo, D.K. and Sento, S. (1991). Synaptic connections of the auditory nerve in cats: relationship between endbulbs of Held and spherical bushy cells. J. Comp. Neurol. 305: 35-48.

Saint-Marie, R.L. and Baker, R.A. (1990). Neurotransmitter-specific uptake of $\left.{ }^{3} \mathrm{H}\right] g l y c i n e$ from the inferior colliculus by ipsilateral projections of the superior olivary complex and nuclei of the lateral lemniscus. Brain Res. 524: 244-253.

Saint-Marie, R.L., Ostapoff, E.M., Morest, D.K., and Wenthold, R.J. (1989). Glycine-imunoreactive projection of the cat lateral superior olive: possible role in midbrain ear dominance. J. Comp. Neurol. 279: 382-396.

Scidana, E. (1993). Onion-like organization of the mamalian dorsal nucleus of the lateral lemniscus. International workshop on Binaural Processing in the Auditory Brainstem, Ottawa, Canada, June 16-19. 
Sally, S.I. and Kelly. J.B. (1988). Organization of auditory cortex in the albino rat: sound frequency. J. Neurophysiol. 59: 1627-1638.

Sally, S.L. and Kelly. J.B. (1992). Effects of superior olivary complex lesions on binaural responses in rat inferior colliculus. Brain Res. 572: 5-18.

Sanes, D.H., Geary, W.A., Wooten, G.F. and Rubel, E.W. (1987). Quantitative distribution of the glycine receptor in the auditory brain stem of the gerbi:. J. Neuroscience $7(11)$ : 3793-3802.

Sanes, D.H. and Wooten, G.F. (1987). Developinent of glycine receptor distribution in the lateral superior olive of the gerbil. J. Neuroscience 7(11): 3803-3811.

Schaefer, K.P. $(1 \overline{9} 70)$. Unit analysis and electrical stimulation in the optic tectum of rabbits and cats. Brain Behav. Evol. 3: 222-240.

Schweizer, H. (1981). The connections of the inferior colliculus and the organization of the brainstem auditory system in the greater horseshoe bat (Rhinolophus ferrumequinum). J. Comp. Neurol. 201: 25-49.

Semple, M.N. and Aitkin, L.M. (1979). Representation of sound frequency and laterality by units in central nucleus of cat inferior colliculus. J. Neurophysiol. 42: 1626-1639.

Semple, M.N. and Aitkin, L.M. (1980). Physiology of pathway from dorsal cochlear nucleus to inferior colliculus revealed by electrical and auditory stimulation. Exp. Bzain Res. 41: 19-28.

Shneiderman, A. and Henkel, C.K. (1987). Banding of lateral superiol oidivaz: nucleus afferents in the inferior colliculus: a possible substrate for sensory integration. J. Comp. Neurol. 266: 519-534.

Shneiderman, A. and Oliver. D.L. (1989). EM autoradiographic study of the projections from the dorsal nucleus of the lateral lemniscus: a possible source of inhibitory inputs to the inferior colliculus. $J$. Comp. Neurol. 286: 28-47.

Shneiderman, A., Oliver, D.L. and Henkel, C.K. (1988). Connections of the dorsal nucleus of the lateral lemniscus: an inhibitory parallel pathway in the ascendiag auditory system? J. Comp. Neurol. 276: i $88-208$.

Smich, F.H. ard Rhode, W.S. (1985). Electron microscopic teatures of chysicicgically choracterized, HRP-labeled fusiform celis in the cat dorsal cochlear nucleus. J. Comp. Neurol. 237: 127-143.

5.rith, P.P. and Rhode, W.S. (1987). Characterization of HFi-labeled globular bushy cells in the cat anteroventral cochlear nucleus. J. Comp. Neurei. 266: 360-375.

Smith, P.h. and Rhode, W.S. (1989). Structural and functional properties distinguish two types of multipolar cells in the ventra? coshlear nucleus. J. Comp. Neurol. 262: 595-616.

Smith, P.H.. Joris, P.X., Carney, L.H. and Yin, T.C.T. (1991). Projecions of physiologiccally characterized globular bushy cell axons from the cochiear nucleus of the cat. J. Comp. Neurol. 304: $387-407$.

Smith, P.A. et al. (1987). Projections of the globular bushy cells in the cat. Neurosci. Abstr. 13: 547.

Spangler. K.M et al. (1985). The projections of principal cells of the medial nucleus of the trapezoid body in the cat. J. Comp. Neurol. $238: 249-262$.

Staatz-Benson, C. and Potashner, S.J. (1987). Uptake and release of glycine in the guinea pig cochlear nucleus. J. Neurochem. 49: $128-$ 137 .

Staatz-Benson, C. and Potashner, S.J. (1988). Uptake and release of glycine in the guinea pig cochlear nucleus after axotomy of afferent or centrifugal fiber. J. Neurochem. 51: 370-379.

Stelzer, A. and Wong, R.K.S. (1989). GABAA-A responses in hippocampal neurens are potentiated by glutamate. Nature 337: 171-173. 
stotler, W.A. (1953). An experimental study of the cells and connections of the superior olivary complex of the cat. J. Comp. Neurol. 98: 401-432.

Strominger, N.L. and Oesterreich, R.E. (1970). Localization of sound after section of the brachium of the inferior colliculus. J. Comp. Neurol. $138: 1-18$.

strominger, N.L. and strominger, A.I. (1971). Ascending brainstem projections of the anteroventral cochlear nucleus in the rhesus monkey. J. Comp. Neurol. 143: 217-242.

Tanaka, K. et al. (1985). The organization of neurons in the nucleus of the lateral lemniscus projecting to the superior colliculi in the rat. Brain Res. 341: 252-260.

Thompson, G.C.. Cortez, A.M. and Lam, D.M. (1985). Localization of GABA immuno-reactivity in the auditory brainstem of guinea pigs. Brain Res. 339: 119-122.

Thompson, G.C. and Masterton, R.B. (1978). Brain stem auditory pathways involved in reflexive head orientation as sound. $J$. Neurophysiol. 41: 1183-1202.

Tolbert, L.P. and Morest, D.K. (1982a). The neuronal architecture of the anteroventral cochlear nucleus of the cat in the region of the cochlear nerve root: Golgi and Nissl methods. Neuroscience I: 30133030 .

Tolbert, L.P. and Morest, D.K. $(1982 \mathrm{~b})$. The neuronal architecture of the anteroventral cochlear nucleus of he cat in the region of the coshlear nerve root: electron microscopy. Neuroscience 7: 30533067.

Tolbert, L.P., Morest, D.K. and Yurgelun-Todd, D.A. (1982). "nhs neuronal architecture of the anteroventral cochlear nucleus of the cat in the region of the cochlear nerve root: horseradish: peroxidase labelling of identified cell types. Neuroscience ?: $3031-3052$.

Tsuchitani, C. (1977). Functional organization of lateral cell groups of cat superior olivary complex. J. Neurophysiol. 40: 296-318.

Tsuchitani, C. and Boudreau, J.C. (1966). Single unit aralj'sis c.f cat superior olive $S$ segment with tonal stimuli. J. iteurofitysiol. z9: $684-697$.

Uchizono, K. (1965). Characteristics of excitatory and inhibitory symapses in the central nervous system of the cat. liat. ire 2j: 542 643.

Yaldivia, 0. (1971). Methods of fixation and the morphology of synaptic vesicles. J. Comp. Neurol. 142: 257-274.

Van Nocrt, J. (1969). The structure and connections of the inferior colliculus. An investigation of the lower auditory system. Van Gcrcum and Co.. N.V.. Netherlands.

Vater, M. and Feng. A.S. (1990). Functional organization of ascendrng and descending connections of the cochlear nucleus of horsesfice bats. J. Comp. Neur 4. 292: 373-395.

Warr. W.B. (1966). Fiber degeneration following lesions in the anterior ventral cochlear nucleus of the cat. Exp. Neurol. i4: 453474 .

Warr. W.B. (1969). Fibex degeneration following lesicns in the posteroventral cochlear rucleus of the cat. Exp. Neirol. 23: 140155.

Warr, W.B. (1972a). Fiber degeneration following lesions in the multipolar and globular cell areas in the ventral cochlear aucleus of the cat. Brain Res. 40: 247-270.

Warr. W.B. (1972b). Olivocochlear and vestibular efferent neurons of the feline brain stem. Their location, morphology, and number determined by retrograde axonal transport and acetylcholinesterase histochemistry. J. Comp. Neurol. 161: 159-182. 
warr, w.B. (1982). Parallel ascending pathways from the cochlear nucleus: neuroanatomical evidence of functional specialization. In: Neff, W.D. (ed.). Contributions to sensory physiology. vol. 7 . Academic Press, New York.

Wenthold, R.J. (1979). Release of endogenous glutamic acid, aspartic acid and GABA from cochlear nucleus slices. Brain Res. 162: 338343.

Wenthold, R.J. (1991). Neurctransmitters of brainstem auditory nucle1. In: R.A. Altschuler, B.M. Clopton, R.P. Bobbin and D.W. Hof fman (Eds.) Neurobiology of Hearing: The Central Auditory System, Raven Press, New York, pp. 121-140.

Wenthold, R.J. and Hunter, C. (1990). Immunocytochemistry of glycine and glycine receptors in the central auditory system. In O.P. Ottersen and $J$. Storm-Mathisen (Eds.) Glycine Neurotransmission, Wiley, New York, Pp. 391-416.

Wenthold, R.J.. Huie, D. Altschuler, R.A. and Reeks, K.A. (1987). Glycine immunoreactivity localized in the cochlear nucleus and superior olivary complex. Neuroscience 22: 897-912.

Wenthold, R.J. and Martin, M.R. (1984). Neurotransmitters of the auditory nerve and central auditory system. In: Berlin, C., ed. Hearing science: recent advances. San Diego: College-Hill Press, pp. 341-369.

Wenthold, R.J. and Morest, D.K. (1976). Transmitter related enzymes in the guinea pig cochlear nucleus. Neurosci. Abst. 2 : 28 .

Wenthold, R.J. et al. (1988). Glycine receptor immunoreactivity in the ventral cochlear nucleus of the guinea pig. J. Comp. Neurol. 276: 423-435.

Whitley, J.M. and Henkel, C.K. (1984). Topographical organization of the inferior collicular projection and other connections of the ventral nucleus of the lateral leminiscus in the cat. J. Comp. Neurol. 229: 257-270.

willard, F.H. and Martin, G.F. (1983). The auditory brainstem nuclel and some of their projections to the inferior colliculus in the North American opossum. Neuroscience 10: 1203-1232.

Villard, F.H. and Ryugo, D.K. (1983). Anatomy of the certral audicory system. In: willott. J.F. (ed.) The auditory psychobuology of the mouse. Thomas, Springfield, pp. 201-304.

Wise, L.ž and Irvine, D.R.F. (1983). Auditory response propertles of neurons in deep layers of cat superior colliculus. J. Neurophysacd. 49: $674-685$.

Wise, I.Z, and Irvine, D.R.F. (2985). Topograptic organization of interaural intensity difference sensitivity in deep layers of cat superior colliculus: implications for auditory spatial representation. J. Neurophysiol. 54: 185-211.

Wooliard, H.H. and Harpman, J.A. (194) . The connections of the inferior colliculus and of the dorsal nucleus of the lateral lemniscus. J. Anat. Lond. 74: 441-457.

Wu, S.H. and Kelly, J.B. (1991). Physiological properties of neurons in the mouse superior olive: membrane characteristics and postsynaptic responses studied in vitro. J. Neurophysiol. Es: 230 246.

W., S.H. and Yelly, J.B. (1992a). MMDA, non-NMDA and glycine receptors mediate binaural interaction in the lateral superior olive: physiological evidence from mouse brain slice. Neurosci. Letters 134: $257-260$.

Wu, S.H. and Kelly, J.B. (1992b). Synaptic pharmacology of the superior olivary complex studied in mouse brain slice. J. Jeurosci. 12(8): $3084-3097$.

Wu, S.H. and Oertel. D. (1986). Inhibitory circuitry in the veritral cochlear nucleus is probably mediated by glycine. J. Neurosc1. 6 : 
2691-2706.

Zarbin, M.A., Wamsley, J.K. and Kuhar, M.J. (1981). Glycine receptor: light microscopic autoradiographic localization with tritiated steychnine. J. Neurosci. 1: 532-547.

zook, J.M. and Casseday. J.H. (1979). Connections of the nuclei of the lateral lemniscus in the mustache bat, Pteronotus parnellii. Neurosci. Abstr. 5: 34.

Zook, J.M. and Casseday, J.H. (1982a). Ozigin of ascending projections to the inferior colliculus in the mustache bat, pteronotus parnelli. J. Comp. Neurol. 207: 14-28.

zook, J.M. and Casseday. J.H. (1982b). Cytoarchitecture of auditory system in lower brainstem of the mustache bat, pteronotus parnellii. J. Comp. Neurol. 207: 1-13.

20ok, J.M. and Casseday. J.H. (1987). Convergence of ascending pathways at the inferior colliculus of the mustache bat, Pteronotus parnelli. J. Comp. Neurol. 261: 347-361.

2vorykin, V.P. (1964). Morphological substrate of ultrasonic and locational capacities in the dolphin. Fed. Proc. 23: T647-T654. 
APPENDIX A

List of Abbreviations

AA

AP

APD

AVCN

CF

CLL

CN

DCN

DMPO

DNLL

EE (EE/E)

EI (EO/I)

EO (EO/O)

HPP

IC

ICC

$I \subseteq P$

ICX

IE

IID

INLI

ITD

LSO

LNTE

MNTB anterior part of anterior division of AVCN

posterior part of anterior division of AVCN posterodorsal part of anterior division of AVCN anterior ventral division of the cochlear nucleus characteristic frequency commissure of the lateral lemniscus

cochlear nucleus

dorsal cochlear nucleus

dorsomedial periolivary nucleus of SOC

dorsal nucleus of the lateral lemniscus

binaural response type - cell excited by stimulation of either ear alone.

binaural response type - cell excited by contralateral stimulation and inhibited by' ipsilateral stimulation.

binaural response type - cell excited by contralateral stimulation, ipsilateral stimulation has no effect.

horseradish peroxidase

inferior colliculus

central nucleus of the inferior colliculus

pericentral nucleus of the inferior colliculus

external nucleus of the inferior colliculus

binaural response type - cell inhibited by contralateral stimulation and excited by jpailatera] stimulation.

interaural intensity difference

intermediate nucleus of the lateral lemniscus

interaural time difference

lateral superior olivary nucleus

lateral nucleus of the :rapezoid body

medial nucleus of the trapezoid body 
MSO

NLL

OE (OE/O)

PD

PV

PVCN

soc

SPO

VCN

VNLI

VNTB medial superior olivary nucleus

nuclei of the lateral lemniscus

binaural response type - cell excited by ipsilateral stimulation, contralateral stimulation has no effect.

dorsal part of posterior division of AVCN

ventral part of posterior division of AVCN

posterior ventral division of the cochlear nucleus

superior olivary complex

superior paraolivary nucleus

ventrai division of the cochlear nucleus

ventral nucleus of the lateral lemniscus

ventral nucleus of the trapezoid body 
Appendix B:

Preparation of Equithesin

Equithesin, which could be obtained previously from Jensen-Salskery Labs, is no longer comercially available, but may be prepared from $21.3 \mathrm{~g}$ chloral hydrate and $4.8 \mathrm{~g}$ pentobarbitol sodium dissolved in 1.54 benzyl alcohol, 408 propylene glycol, 108 ethanol, 2.58 sodium benzoate, 2.58 benzoic acid and 43.58 distilled $\mathrm{H}_{2} \mathrm{O}$ to make $500 \mathrm{~mL}$, with pH adjusted to 6.7 using sodium hydroxide. 
Appendix C:

Figure 1. Composite reconstruction to illustrate the locations of units with characteristic frequencies in the lemniscal nuclei (same reconstruction as in Figure 5, but all lemniscal nuclei have been included). 


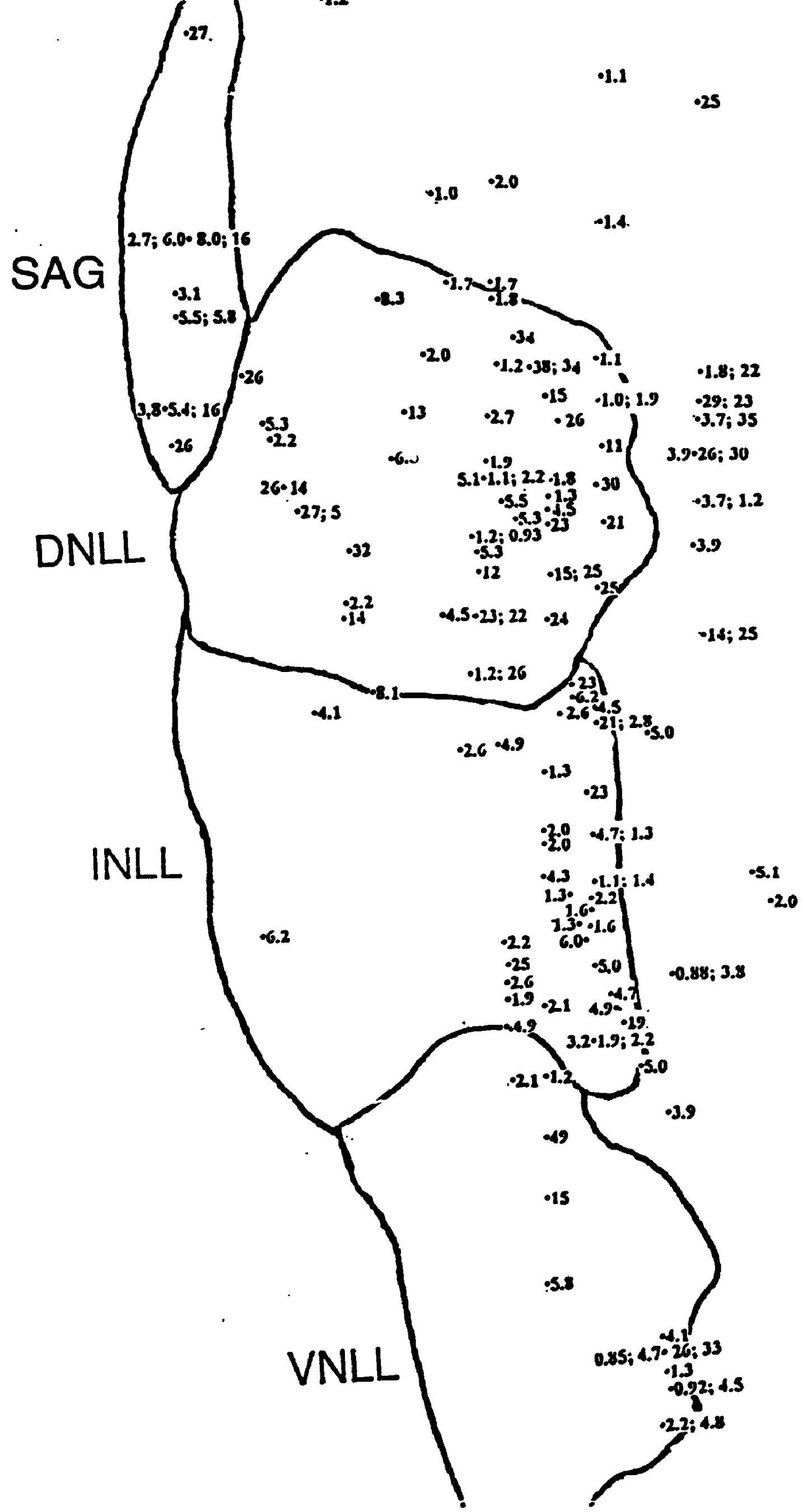


Appendix D:

Figure 2. Post-stimulus histograms for two well-isolated single units recorded from DNLL, illustrating the effects of different sound pressure levels and frequencies of tone burst (binaural or monaural) stimulation. 
BUS6-2; CF = $4.60 \mathrm{kHz}$

EO/O Oneet chopper whth sustained component at highest sound preseure levels;

Contraluteral sound only; $1.38 \mathrm{kHz}$ tone burst for varying sound pressure levels.

Plots with expanded scales shown at left.
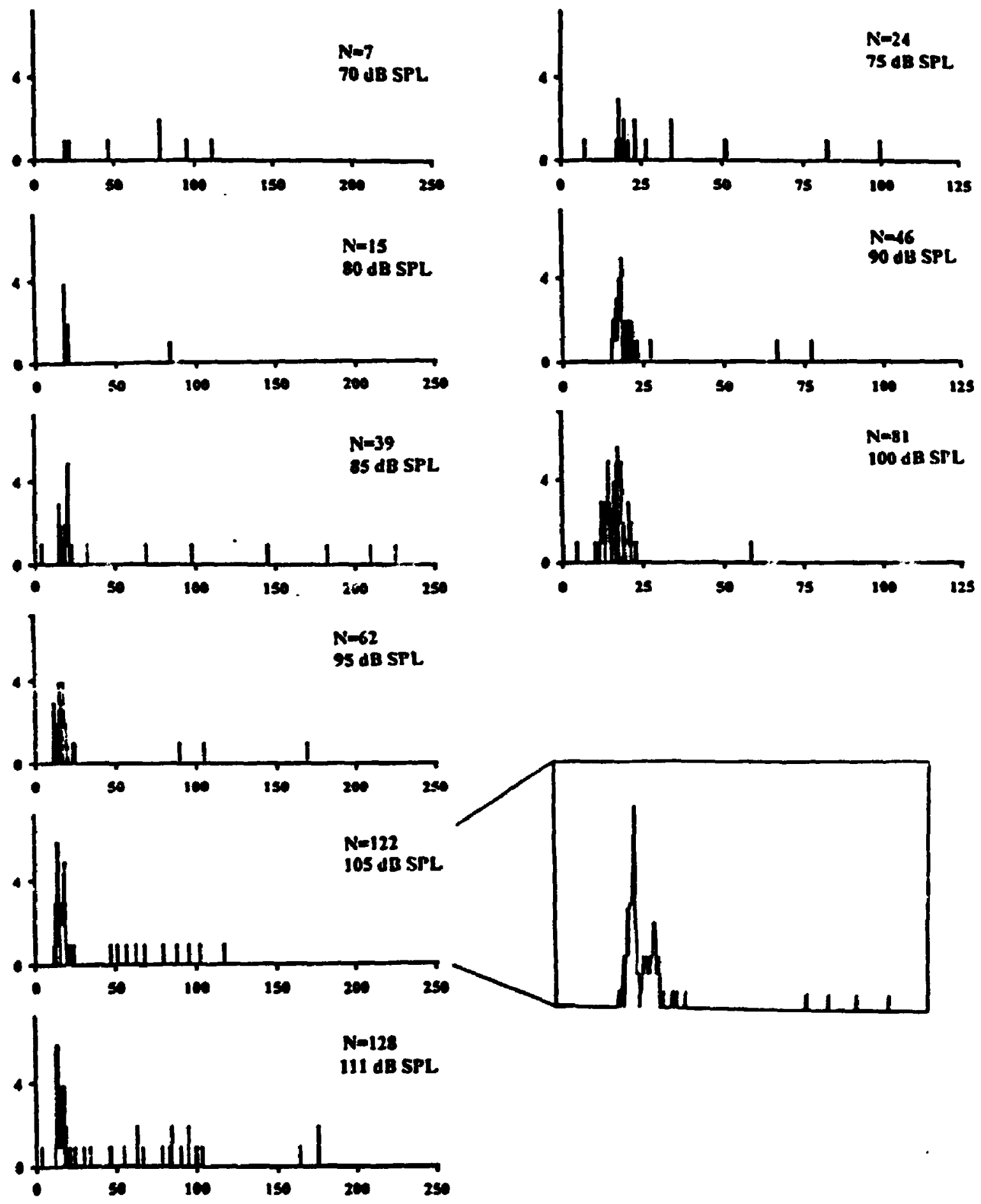
BUS3-2; CF $=4.80 \mathrm{kHz}$

EONO Oneet chopper with sustalned component at higheat sound presesure levels:

Contraleteral sound only; $2.27 \mathrm{kHz}$ tone burst for varying sound presesure levels.
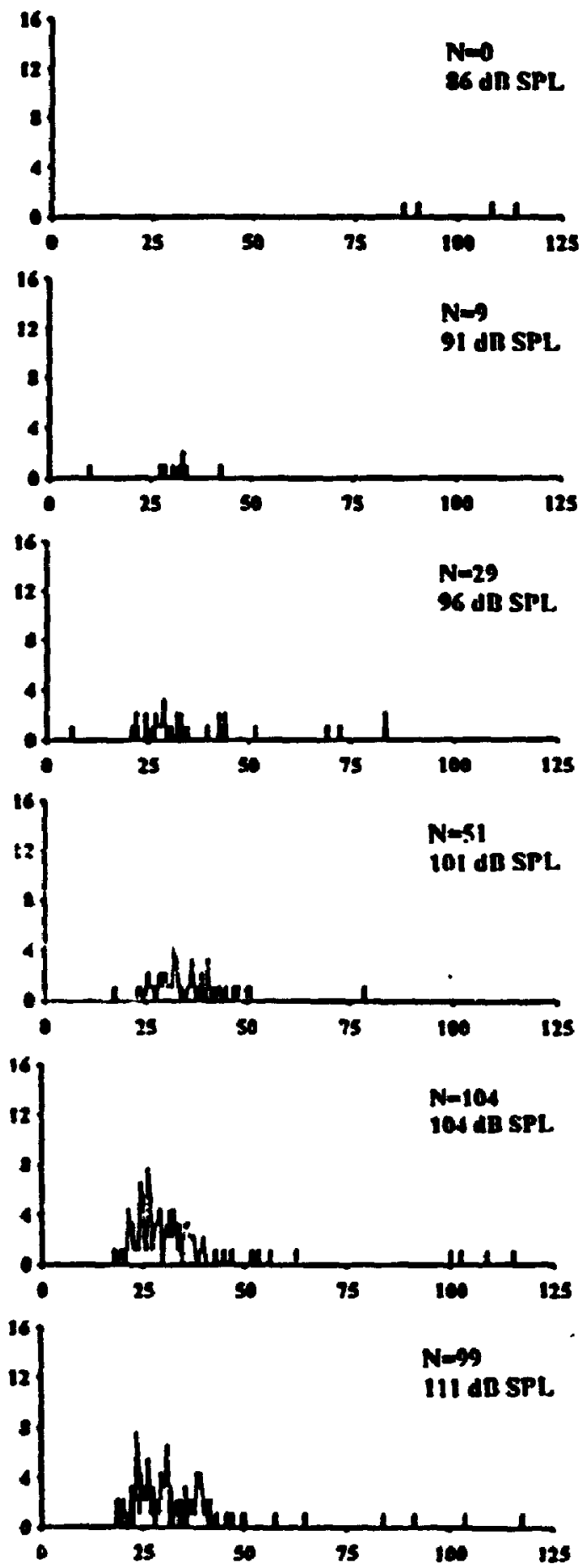

Contralateral sound only; $3.23 \mathrm{kHz}$ tone burst for varying sound pressure levels.
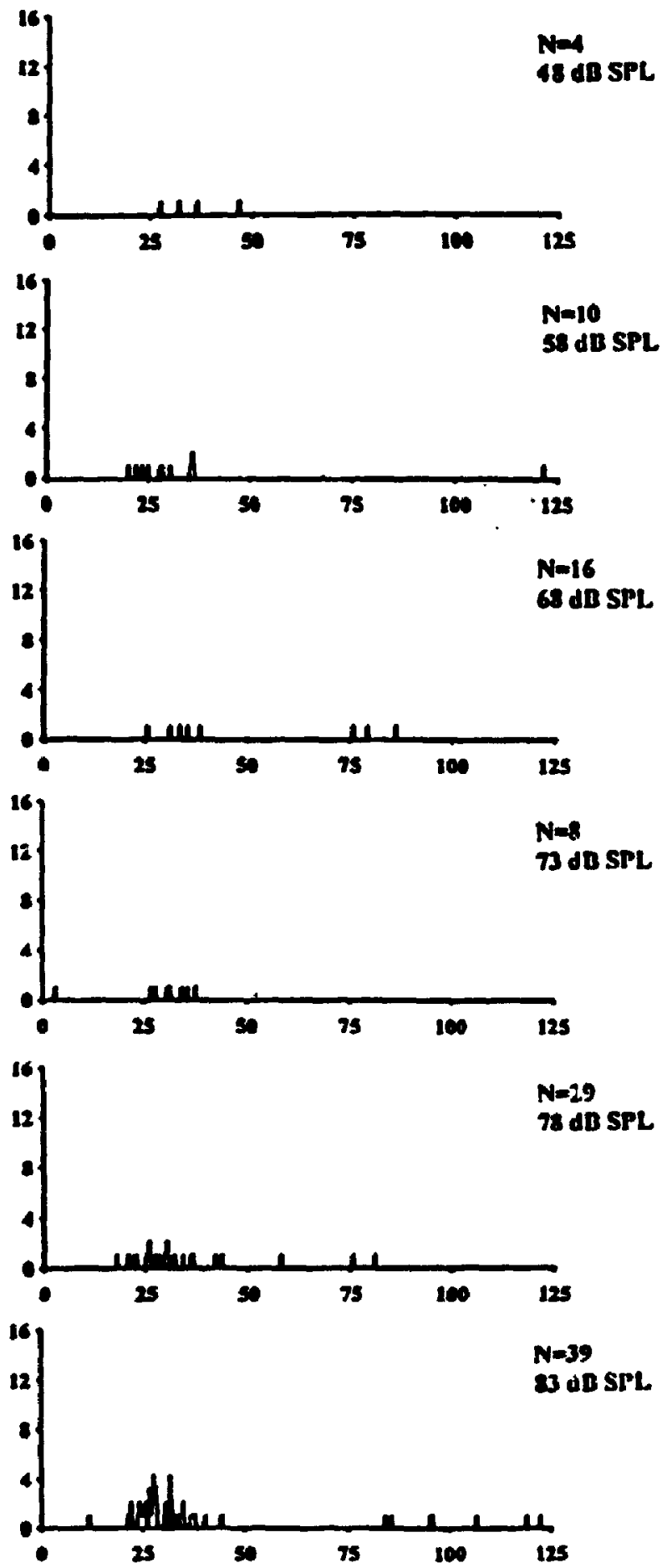
BUse-2; CF $=4.50 \mathrm{kHz}$

EONO Onset chopper with sustained componemt at hlgheet sound pressure levels;

Contralateral sound only; $1.77 \mathrm{kHz}$ tone burst for varying sound pressure levels (plote with entarged scales at lent).
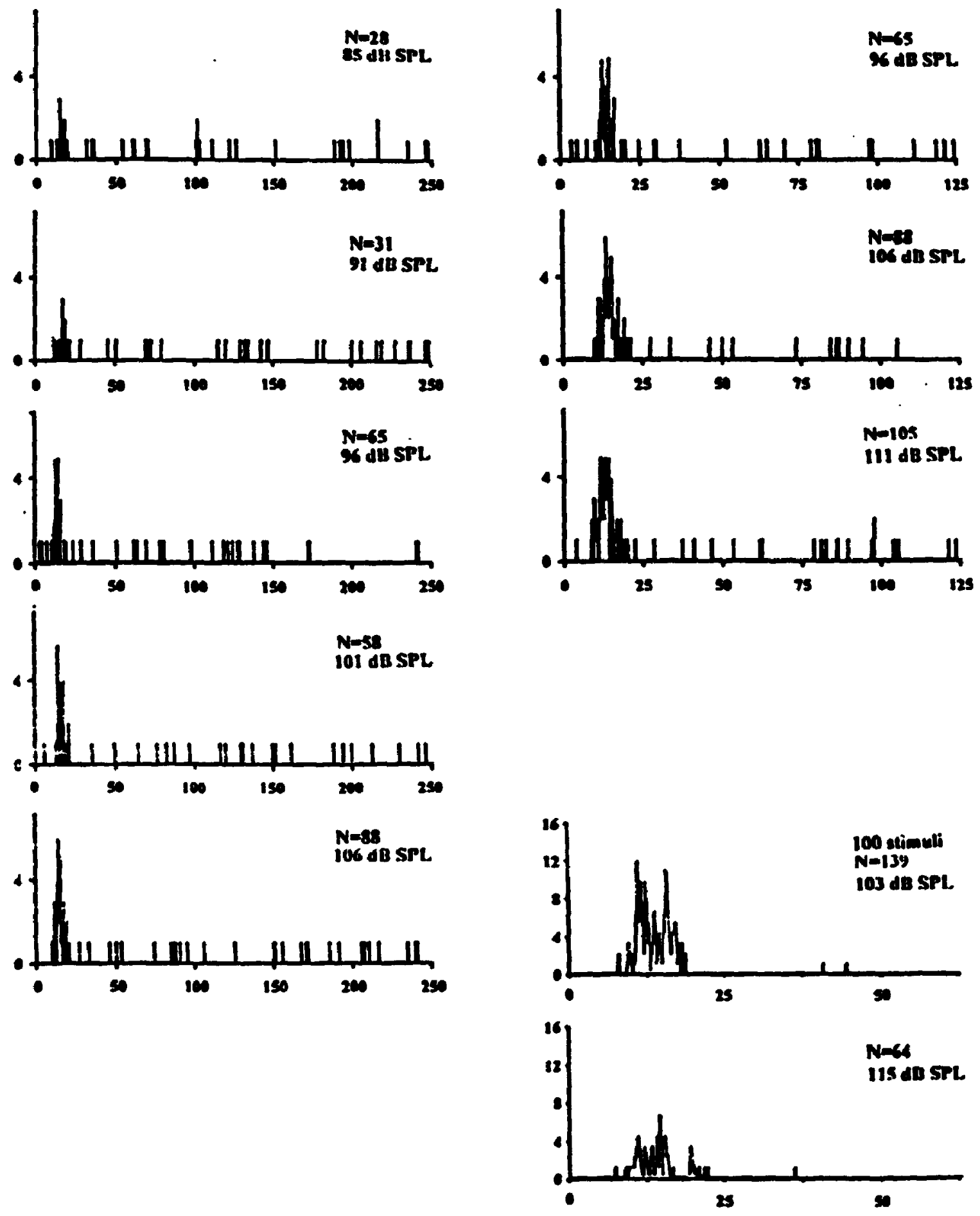


\section{BUS8-2; CF - $4.80 \mathrm{kHz}$}

EOIO Oneet chopper with sustained component at highest sound pressure levels;

Contralateral sound only; $4.31 \mathrm{kHz}$ lone buret for varying sound pressure levels.

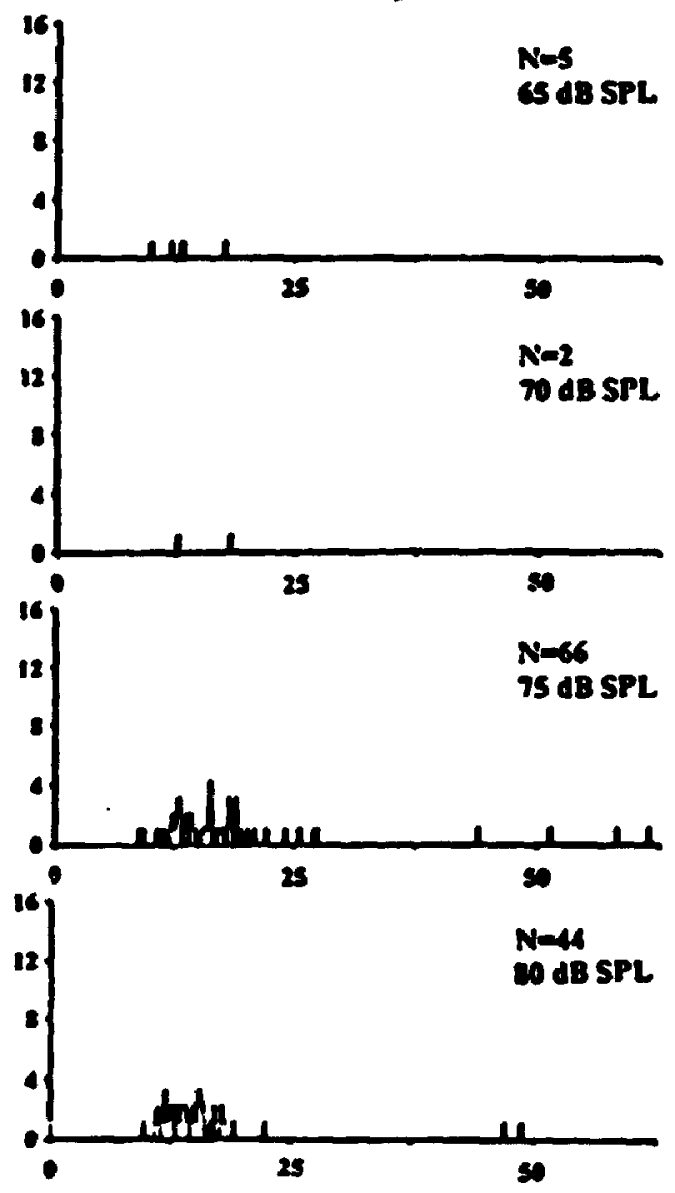


BUS8-2; CF = $4.60 \mathrm{kHz}$

EOrO Oneet chopper with sustained componemt at highest sound preseure levels:

Contralateral sound only; $1.00 \mathrm{kHz}$ tone burat for vanjing sound preseure levels .
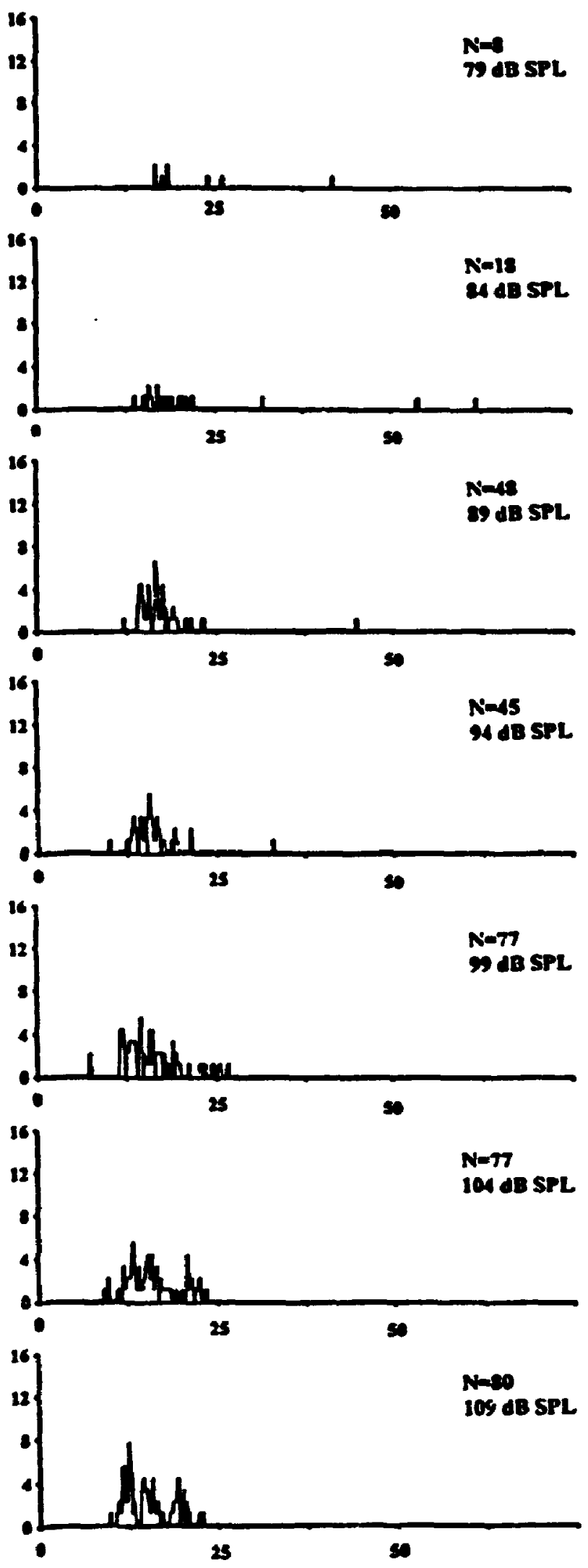
BU52-1; CF = $8.32 \mathrm{kHz}$ EOn Oneat chopper

Contralaterel sound only; 8.12 kHz tone burst for varying sound pressure levels.
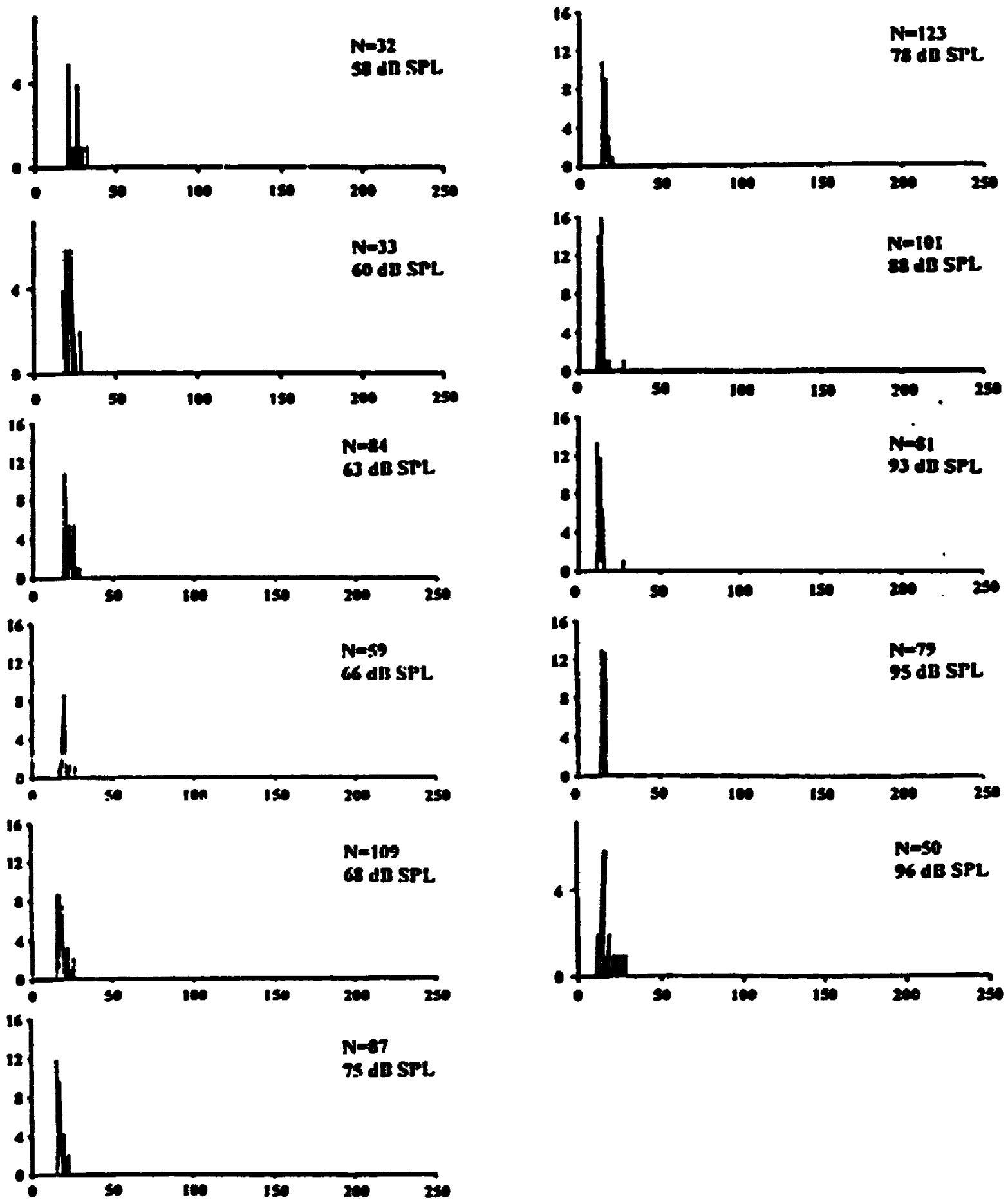
BUS2-1; CF = $8.32 \mathrm{kHz}$ EOh Onset chopper

Contralateral sound only; $\mathbf{8 . 1 2} \mathrm{kHz}$ tone buret for varying sound pressure levels.
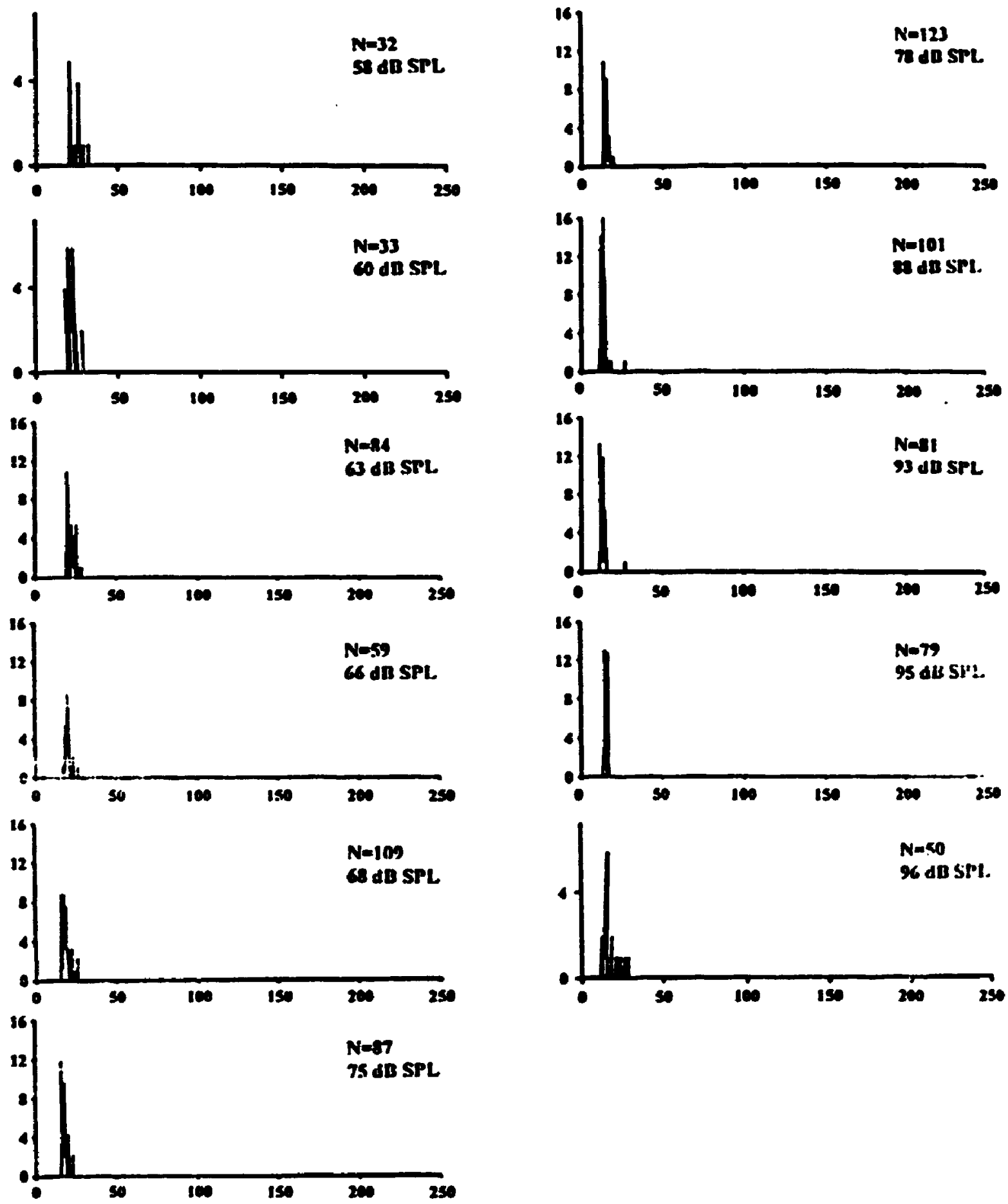
BUS2-1; CF - $8.32 \mathrm{kHz}$ EON Onzet chopper

8.12 litiz tone burst

Contralceral sound pressure lovel held constant at 73 dB;

tpolleteral sound preseure level varled.
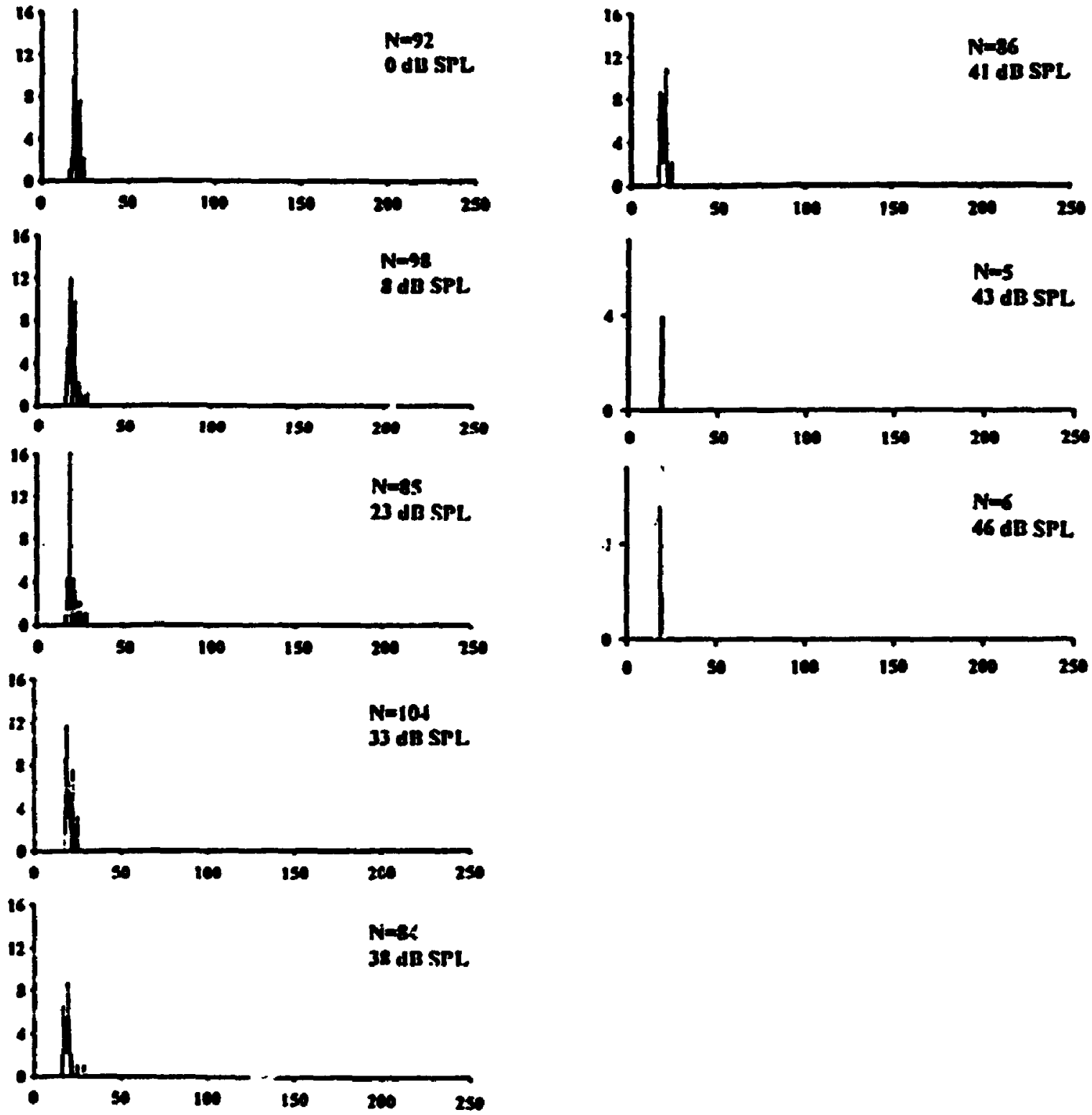
142

BU52-1; CF $=8.32 \mathrm{kHz}$ EON Onset chopper

Contraleteral sound only:

Different frequencies of lon burst stimulation for similar sound pressure levels.
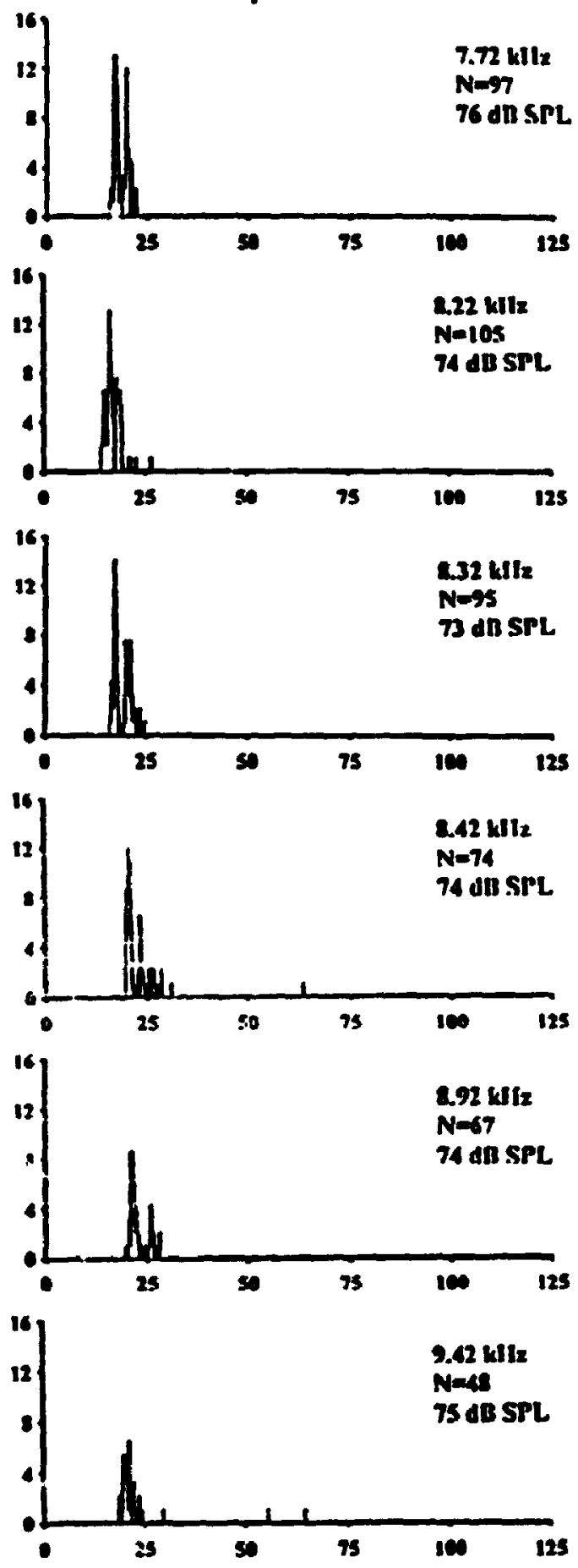

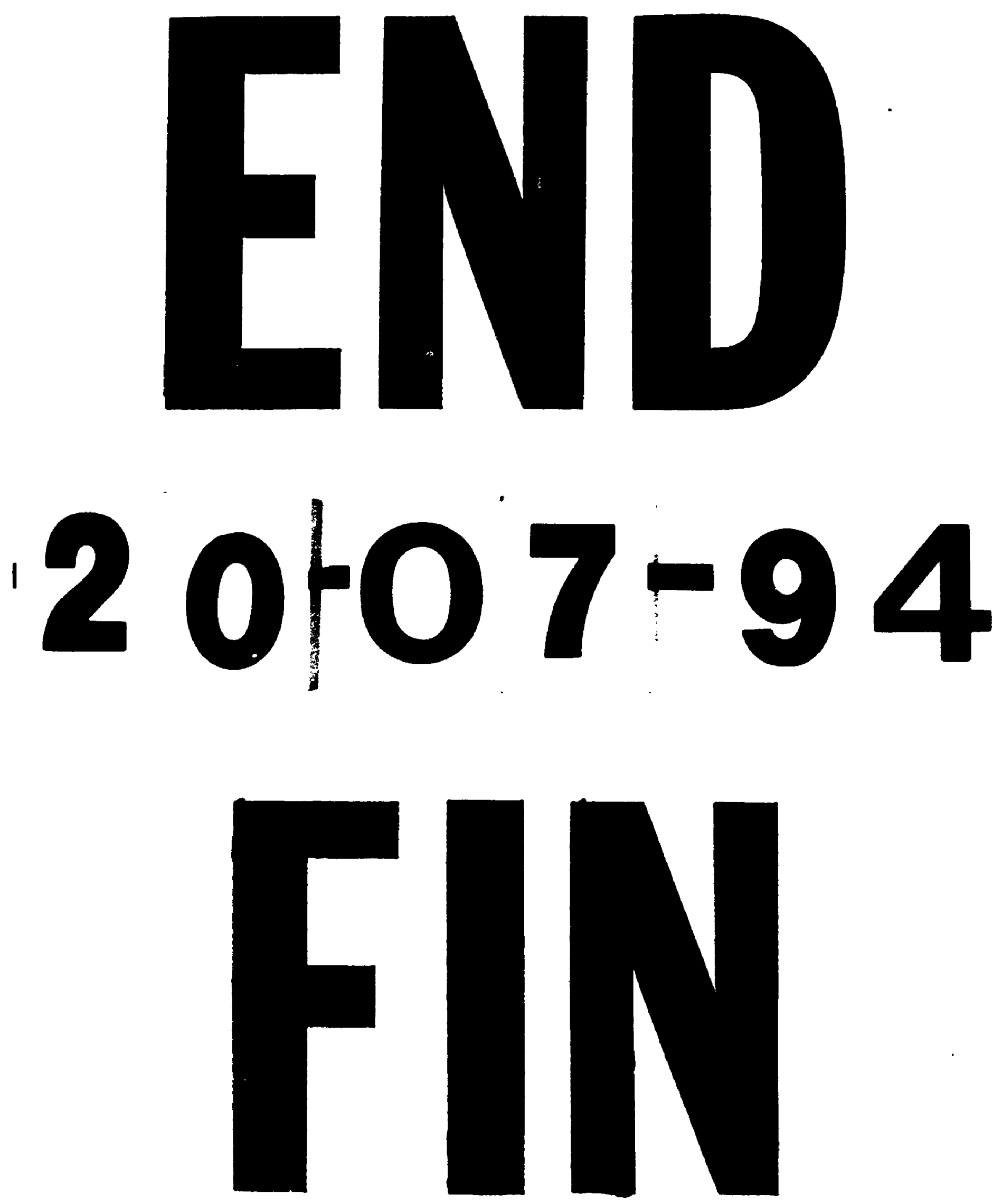